\title{
MECHANISTIC UNDERSTANDING OF MICROBIAL PLUGGING FOR IMPROVED SWEEP EFFICIENCY
}

\section{Final Scientific/Technical Report}

Oct 1, 2004 - September 30, 2008

\author{
Steven Bryant \\ Larry Britton
}

December 2008

\section{DE-FC26-04NT15524}

The University of Texas at Austin

1 University Station

Austin, TX 78712-0228 


\section{DISCLAIMER}

This report was prepared as an account of work sponsored by an agency of the United States Government. Neither the United States Government, nor any agency thereof, nor any of their employees, makes any warranty, express or implied, or assumes any legal liability or responsibility for the accuracy, completeness, or usefulness of any information, apparatus, product, or process disclosed, or represents that its use would not infringe privately owned rights. Reference herein to any specific commercial product, process, or service by trade name, trademark, manufacturer, or otherwise does not necessarily constitute or imply its endorsement, recommendation, or favoring by the United States Government or any agency thereof. The views and opinions of authors expressed herein do not necessarily state or reflect those of the United States Government or any agency thereof. 


\begin{abstract}
Microbial plugging has been proposed as an effective low cost method of permeability reduction. Yet there is a dearth of information on the fundamental processes of microbial growth in porous media, and there are no suitable data to model the process of microbial plugging as it relates to sweep efficiency. To optimize the field implementation, better mechanistic and volumetric understanding of biofilm growth within a porous medium is needed. In particular, the engineering design hinges upon a quantitative relationship between amount of nutrient consumption, amount of growth, and degree of permeability reduction. In this project experiments were conducted to obtain new data to elucidate this relationship.

Experiments in heterogeneous (layered) beadpacks showed that microbes could grow preferentially in the high permeability layer. Ultimately this caused flow to be equally divided between high and low permeability layers, precisely the behavior needed for MEOR. Remarkably, classical models of microbial nutrient uptake in batch experiments do not explain the nutrient consumption by the same microbes in flow experiments. We propose a simple extension of classical kinetics to account for the selflimiting consumption of nutrient observed in our experiments, and we outline a modeling approach based on architecture and behavior of biofilms. Such a model would account for the changing trend of nutrient consumption by bacteria with the increasing biomass and the onset of biofilm formation. However no existing model can explain the microbial preference for growth in high permeability regions, nor is there any obvious extension of the model for this observation. An attractive conjecture is that quorum sensing is involved in the heterogeneous bead packs.
\end{abstract}




\section{TABLE OF CONTENTS}

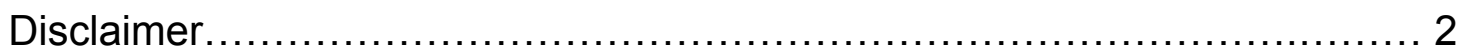

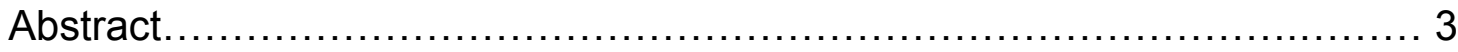

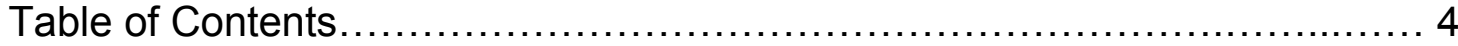

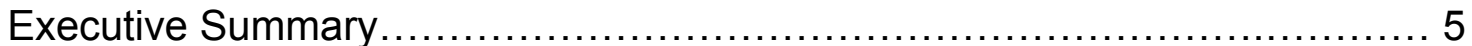

Results of Work During Reporting Period .................................... 6

Topic 1: Experimental Evaluation of Microbe Growth and Permeability

Reduction........................................................6

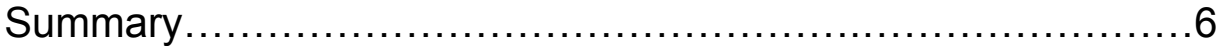

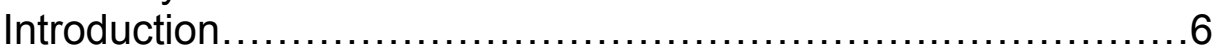

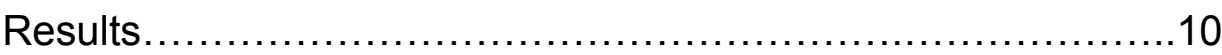

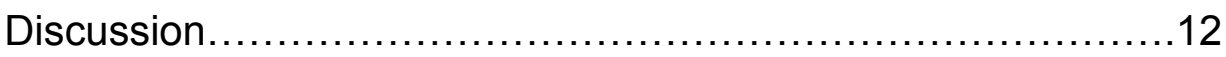

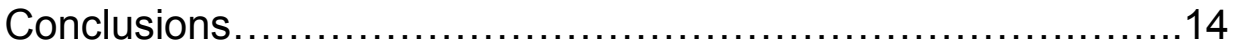

Topic 2: Modeling of Microbe Growth and Permeability Reduction.............. 59

Summary........................................................ 59

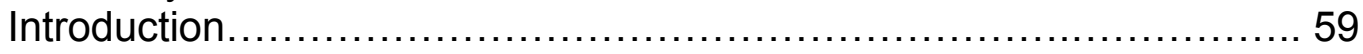






\section{DE-FC26-04NT15524}

\section{MECHANISTIC UNDERSTANDING OF MICROBIAL PLUGGING FOR IMPROVED SWEEP EFFICIENCY}

Period: Oct 1, 2004 - Sept 30, 2008

\section{EXECUTIVE SUMMARY}

Among the many mechanistic explanations of MEOR, simple plugging of thief zones by biomass (biofilm) growing on injected or in-situ substrates is the most plausible and also the most straightforward, achievable approach. Yet there is a dearth of information on the fundamental processes of microbial growth in porous media, and there are no suitable data to model the process of microbial plugging as it relates to sweep efficiency. This research seeks to understand microbial growth in porous media and to identify parameters that can be used to model and predict the degree of microbial plugging necessary to improve sweep efficiency for incremental oil production in U.S. fields. The approach can be explained as the melding of the science of biofilm research with reservoir engineering design, the overall objective being to develop a quantitative procedure for assessing the likelihood of incremental oil production by microbial plugging of thief zones.

The most important result from this research was the observation that microbes could grow preferentially in a high permeability layer, and by so doing cause flow to be equally divided between high and low permeability layers. This behavior is ideal for MEOR application. This research showed that an extension of classical kinetics could be introduced that accounts for the self-limiting consumption of nutrient observed in our experiments. The preference for growth in high permeability regions cannot be explained, however, and establishes an important question to be addressed by future research. This report describes the experiments performed (Topic 1) and the analysis of them (Topic 2). 


\section{RESULTS OF WORK DURING REPORTING PERIOD}

\section{Topic 1: Experimental Evaluation of Microbe Growth and Permeability Reduction}

\section{Summary}

Microbial plugging has been proposed as an effective low cost method of permeability reduction. To optimize the field implementation, better mechanistic and volumetric understanding of biofilm growth within a porous medium is needed. In particular, the engineering design hinges upon a quantitative relationship between amount of nutrient consumption, amount of growth, and degree of permeability reduction. As a first step toward such a relationship, a Pseudomonas aeruginosa culture was inoculated into columns of glass beads and Berea sandstone cores. A growth substrate with acetate as carbon and energy source was injected continuously. Growth substrate utilization and permeability changes were used to track growth, and post-experiment in situ staining of biomass provided visual evidence of colonization and growth. Growth was observed as grainy coatings but in a spatially complex and unpredictable manner. Permeability was reduced noticeably in each experiment, but replicate experiments exhibited different growth rates and ultimate growth-induced permeability reduction. The experiments demonstrated that microbial growth is effective for reducing flow in porous media. Obtaining a mechanistic interpretation of the behavior will require a better understanding of the variability in microbe growth at the grain scale.

\section{Introduction}

United States onshore oil fields have undergone extensive waterflooding. Many fields reach an economic limit at high watercuts because of the increased costs of lifting large amounts of fluids with only a small amount of produced oil. In many cases, high permeability thief zones contribute much of the produced water. Bypassed oil remaining in the lower permeability reservoir rock can be mobilized if injected fluids are effectively diverted from the thief zones to the remaining areas of the reservoir. Microbial plugging has been touted as a low cost, potentially high reward technology for increasing sweep efficiency by occluding thief zones in mature oil fields. Stimulating the growth of indigenous microbes is a particularly attractive version of this idea. This circumvents the twin challenges of cultivating a strain that will thrive at reservoir conditions and of propagating organisms to useful distances within the formation. This approach has been implemented with technical and economic success in the North Blowhorn Creek Unit (Vadie et al, 2002).

Quantitative modeling of the behavior of this and other microbial EOR techniques is difficult. In particular the relationship between the rate of consumption of microbial nutrients (e.g. acetate, dissolved oxygen) and the mechanism of permeability reduction 
(e.g. incremental growth of biofilms on all grain surfaces vs. local growth of colonies that block pores) is not well understood.

The aim of this study is to quantify biofilm induced permeability reductions for a model microbe in porous media columns. Permeability reductions were measured during flow experiments employing a single, well-characterized microbial species growing in a defined growth medium. Carbon source utilization and flow pressures were monitored concurrently. Images of the biofilms within the porous media were acquired by two methods to better understand biofilm location within the pores. Traditional batch growth experiments (no porous media) were conducted to determine growth kinetics independently of the flow experiments.

\section{Previous Work}

Microbial induced permeability reductions in porous media have been verified on several fronts within many fields of science. Most experiments have shown that permeability reductions are normally between $65 \%$ and $95 \%$. Cunningham and Characklis (1991) created a series of experiments in which several porous media of large permeability (between 98 and 2127 Darcy) were inoculated with Pseudomonas aeruginosa and permeability reduction was measured as biofilm grew. After growth was complete each sample had a permeability in the range of 3 to 7 Darcy. Their results indicated that final biofilm-altered permeabilities were similar, despite large differences in original permeability.

Vandevivere and Baveye (1992) conducted flow experiments with Anthrobacter sp. strain AK19, a common soil organism found in areas of gas seepage. The second was $\mathrm{SLI}^{-}$, which is a mutant strain of a groundwater isolate. Sand and glass bead packs with particle diameters ranging from 63 to 125 um were used for the flow experiments. The AK19 induced growth reduced the hydraulic conductivity by $99 \%$. The SLI' experiments showed less than a $50 \%$ reduction in the hydraulic conductivity.

Dunsmore and Lappin-Scott (2004) conducted experiments with Desulfovio EX265. In a 24 hour period permeability was reduced from $9.9 \mathrm{D}$ to $4.9 \mathrm{D}$. This was done on an etched glass plate and biofilm protrusion from the glass was photographed with time. This imaging showed growth, but not fully within a porous media.

Lee and Bae (1998) experimented with 1" diameter by 2" long Berea cores and the Salton-1 microorganism. Their focus was to optimize nutrient concentrations in the growth medium. Salt concentrations and $\mathrm{pH}$ significantly altered the way the films sustained themselves. Salt near $4 \%$ by weight and neutral $\mathrm{pH}$ were favorable for biofilm growth and sustained permeability reductions.

Various attempts have been made to model biofilm growth. Willamson and McCarty (1976) proposed a mechanistic model dependent on a single limiting substrate. This 
model assumes that the rate of substrate diffusion through the biofilm is determined by Fick's law of diffusion and the rate of utilization is given by Monod kinetics. The Rittmann and McCarty model (1980) built on the initial model and introduced the term $\mathrm{S}_{\min }$, the minimum amount of substrate required for biofilm sustenance. Hence the Monod kinetics are only valid for substrate concentrations greater than $S_{\min }$.

Chang et al. (1991) also explored biofilm growth in one-dimensional laboratory corefloods. They incorporated terms for dispersion, convection, growth and decay. The growth in the core was modeled using Monod kinetics. They concluded that mathematical models developed from laboratory corefloods can be successfully used to determine field-scale effects.

\section{Experimental Procedures}

Small columns were constructed of glass bead packs or Berea sandstone. The bead pack columns utilized 38-43 $\mu \mathrm{m}$ glass beads sieved 8-10 times to insure homogeneity. This particular bead size was selected so that pressure drop could be monitored in the flowthrough system. The beads were poured at a slow rate through an hourglass into a $1 \mathrm{~cm}$ dia. by $15 \mathrm{~cm}$ long glass chromatographic column, and the column was vibrated continuously for uniform packing of the beads. The measured porosity of this packing was $35-38 \%$.

Berea sandstone cores, 1 inch dia. by 2 inches long were equipped with machined polycarbonate end units, and then the entire column apparatus was embedded in epoxy. The porosity of these sandstone columns was $19 \%$.

The flow-through system (Figure 1) begins with a FMC adjustable rate pump operated at $1.28 \mathrm{~mL} /$ hour which is roughly equivalent to water velocity in a typical field water flood. The flow-through system tubing is composed exclusively of PTFE chosen for its inertness and the decreased ability for biofilm to adhere to its surface. Pressure monitoring utilized Validyne P2 (0-15 PSI) pressure transducers. Pressure-sensing, voltage signals from the transducers were recorded every 9 seconds using a data acquisition card and NI Datalogger software. The entire system except for the porous media was maintained at $44^{\circ} \mathrm{C}$ which effectively prevented growth of the Pseudomonas aeruginosa $\mathrm{PAO}-1$, biofilm test culture. The porous media column was maintained at 23$24^{\circ} \mathrm{C}$ whereas the remainder of the flow-path (pump, transducers and tubing) were in the oven. These temperatures limited the growth of the test microorganism to the porous media column and not in the other parts of the apparatus.

Porous media columns were flooded to remove all air in pore spaces. First, a vacuum was placed on the porous media followed by the injection of $\mathrm{CO}_{2}$ at 20 PSI. The $\mathrm{CO}_{2}$ was then vacuum evacuated, and the process was repeated several times. While still under a vacuum, the column materials were rapidly saturated with Dworkin Foster mineral medium (Dworkin and Foster 1958). As fluid is pumped through the system, any residual 
$\mathrm{CO}_{2}$ is dissolved and the porous media is saturated. The entire apparatus was sterilized prior to growth experiments by flowing $70 \%$ isopropanol, followed by several volumes of sterile Dworkin Foster growth medium. All fluids introduced to the system were sterilized in an autoclave or filter sterilized. Extreme care was taken to ensure that only the test microorganism was present in the system.

The porous medium was inoculated with a low concentration $\left(\sim 10^{7} \mathrm{cfu} / \mathrm{mL}\right)$ of the Pseudomonas aeruginosa culture. Concentrations above $10^{8}-10^{9} \mathrm{cfu} / \mathrm{mL}$ will experience significant face plugging on the injection side. Indigenous microbes are presumably distributed fairly uniformly within the formation. Inoculation may not establish the same degree of uniformity within the column, but it does provide a controlled means of measuring nutrient consumption and permeability reduction simultaneously. We expect that the intrinsic (local) relationship between consumption, growth and permeability reduction inferred from these experiments will be applicable to other situations.

After inoculation of the column, effluent liquid was monitored for acetate consumption. Acetate was the sole source of carbon and energy, and its depletion was an indicator of microbial growth. Acetate was introduced to the system at a concentration of $700 \mathrm{ppm}$ during the inoculation stage. Residual acetate was measured in small, filter-sterilized liquid samples using gas/liquid chromatography with flame ionization detection. Pressure was continuously monitored via the transducers during the experiment as an indicator of microbial plugging in the column.

Once the pressure drop across the column stabilized ( $40 \mathrm{PV})$, the experiment was concluded. The Pseudomonas aeruginosa attached growth could be dyed effectively using either a nitroblue tetrazolium (NBT) or triphenyl tetrazolium (TTC) solution. These tetrazolium compounds are often used to assess cellular oxido-reductase activities by reducing the soluble tetrazoliums to highly-colored, insoluble formazan compounds. The addition of black or red coloration to the biofilms permits microscopic examination. Once the cellular growth was "stained" with the formazans, the column was carefully injected with a $15 \%$ solution of acrylamide cross-linked with $0.4 \%$ bis-acrylamide and allowed to polymerize. The solidified glass bead/acrylamide was extruded from the glass column, cut into thin slices and viewed with an optical microscope.

Berea sandstone columns were operated similarly to the glass bead columns except that visualization of growth required X-ray techniques. To this end, the columns were examined using High Resolution X-ray Computed Tomagraphy (HRXCT) after staining.

In batch experiments the growth of P. aeruginosa PAO-1 in the liquid medium was measured. The objective was to obtain growth parameters that can be independently used to validate the growth in the columns. The cultures were grown in an oxygen rich, sterile environment in batch in 250-mL Erlenmeyer flasks using 100-mL of Dworkin Foster medium (brine) and about 700ppm acetate as the energy source. At each collection interval, two samples were collected, one of which was run through a gas chromatograph 
to obtain acetate consumption and the other was diluted and plated on growth medium to obtain the number of colony forming units per milliliter $(\mathrm{cfu} / \mathrm{mL})$. The growth curves exhibited classical progression from a lag phase to a period of exponential growth leading to a stationary phase.

It was anticipated that the growth curves would follow classic Monod kinetics and be of the form:

$$
\begin{aligned}
& \mathrm{m}=\mathrm{m}_{\max } \mathrm{A} /\left(\mathrm{K}_{\mathrm{S}}+\mathrm{A}\right) \\
& \mathrm{r}_{\mathrm{B}}=\mathrm{dB} / \mathrm{dt}=\mathrm{mB} \\
& v(\text { acetate }) \rightarrow(\text { cells }) \\
& \mathrm{dA} / \mathrm{dt}=-1000 / v \mathrm{~dB} / \mathrm{dt}
\end{aligned}
$$

where

$$
\begin{array}{ll}
\mathrm{m} & =\text { specific growth rate }\left(\mathrm{hr}^{-1}\right) \\
\mathrm{m}_{\max } & =\text { maximum specific growth rate }\left(\mathrm{hr}^{-1}\right) \\
\mathrm{A} & =\text { acetate concentration }(\mathrm{ppm}) \\
\mathrm{K}_{\mathrm{S}} & =\text { half saturation constant }(\mathrm{ppm}) \\
\mathrm{r}_{\mathrm{B}} & =\text { rate of biofilm generation }(\mathrm{cfu} / \mathrm{mL}-\mathrm{hr}) \\
\mathrm{B} & =\text { microbe concentration }(\mathrm{cfu} / \mathrm{mL}) \\
v & =\text { stoichiometric constant }(\mathrm{cfu} / \mathrm{mg} \text { acetate })
\end{array}
$$

and the factor of 1000 in Eq. (4) converts liters to $\mathrm{mL}$.

Outliers from the growth curve were attributed to cell clumping which is common in stirred liquid cultures of the $P$. aeruginosa. Our objective is to capture the general behavior of the nutrient consumption/cell growth history, so we determined best-fit curves for the acetate vs. time data and cell count vs. time data. These "average behavior" curves were used for modeling the kinetics.

\section{Results}

Flow Experiments. Before inoculation of the porous media, permeability was measured for each sample with a constant flow of single phase Dworkin Foster medium without acetate. After the inoculation of each porous medium, permeability was measured with Dworkin Foster medium as the flowing phase. No difference in permeability was observed before and after inoculation. This means that (1) media with and without acetate have the same viscosity, and (2) inoculated samples with and without organisms have the same permeability. This implies very clearly that the organisms must grow to reduce permeability, and do not reduce it merely by being introduced to the core. Any significant pressure drop in these experiments must be due to microbial growth. 
The formation of biofilm in the bead pack columns is signaled by a rise in inlet pressure to maintain the constant $1.28 \mathrm{~mL} / \mathrm{hr}$ flow. Pressure readings and acetate concentrations in the column effluent were both followed as indicators of biofilm formation. Results of this and the other experiments are summarized in Table 1. Bead pack 1 in which $\phi=$ $39.4 \%$ and $\mathrm{K}_{\mathrm{o}}=1483 \mathrm{md}$ had an overall permeability reduction (measured as $\mathrm{K} / \mathrm{K}_{\mathrm{o}}$ ) of approximately $44 \%\left(\mathrm{~K} / \mathrm{K}_{0}=0.56\right.$, after $45 \mathrm{PV}$, or about 150 hours; Figure 2$)$. A rapid drop in effluent acetate concentration was observed soon after the start of the experiment. This early stage of acetate consumption corresponded to a decrease in permeability of about $15 \%$. The acetate concentration then increased steadily until about $30 \mathrm{PV}$, indicating a slower rate of consumption by microbes, while permeability remained essentially constant. At $30 \mathrm{PV}$ acetate concentration decreased slightly. This coincided with a large reduction in permeability. The permeability and effluent acetate concentration remained unchanged after $\sim 40 \mathrm{PV}$. The intervals over which permeability does not change are interpreted as periods of no further multiplication of microbes, and a steady catabolism of the acetate by the existing biofilm. In other words, the amount of growth had reached a maximum, but substrate (acetate) was still being consumed. The decrease in permeability at $30 \mathrm{PV}$ remains to be explained; see the discussion below.

Bead pack 1 was dyed with a TTC solution to stain the biofilms a red color (Figure 3). A $2-3 \mathrm{~cm}$ thick red ring was observed in the beads at the influent side of the bead pack. The reactants were forced through the core for an extended period of time to encourage all biofilms to reduce the TTC solution and turn red, but the influent edge was the only location where visible staining of the biofilm occurred. It is not clear whether microbes were not established in other parts of the pack during inoculation, or whether growth was restricted in those parts. The possibility remains that TTC downstream of the influent edge was not reduced, and therefore growth was not visible.

Bead pack 2 in which $\phi=38.8 \%$ and $\mathrm{K}_{\mathrm{o}}=1414 \mathrm{md}$ had an overall permeability reduction of $54 \%\left(\mathrm{~K} / \mathrm{K}_{\mathrm{o}}=0.46\right.$; Figure 4$)$. Most of the reduction happened rapidly, within the first two PV, followed by a slower rate of reduction until $13 \mathrm{PV}$ injected. The permeability reduction fluctuated between $54 \%$ and $48 \%$ for the remainder of the experiment ( $45 \mathrm{PV}$ ). Effluent acetate concentration fluctuated around 600 ppm for the first $20 \mathrm{PV}$, then declined steadily to the end of the experiment (40 PV). However, the permeability remained roughly constant during the period of increased acetate consumption. The column was dyed with NBT which stains dark black. Formazan production was not as intense as with TTC in bead pack 1 .

Bead pack $3\left(\phi=38.55 \%\right.$ and $\left.\mathrm{K}_{\mathrm{o}}=1375 \mathrm{md}\right)$ had an overall permeability reduction of $20 \%\left(\mathrm{~K} / \mathrm{K}_{\mathrm{o}}=0.80\right.$; Figure 5$)$ which was much less than in the other experiments. This reduction occurred quickly, within the first 5 PV. No further reduction occurred. Effluent acetate concentration also dropped rapidly at the beginning of the experiment and leveled off at the same point as permeability reduction. Acetate consumption in this experiment was very similar to consumption in bead pack 1 , but qualitatively different from that in bead pack 2 . 
Before dismantling bead pack 3 by extrusion, it was stained black by injecting NBT solution. Growth was observed at both influent and effluent ends of the column, but no staining was seen in the middle. Low magnification microscopy (100X) showed sparse clusters of colonization that was evident from the black colorization (Figure 6). Imaging of individual beads at higher magnification $(500 \mathrm{X})$ revealed the growth as grainy patches on the beads (Figure 7). The generally sparse distribution of microorganisms within the beads seen in Figure 6 was consistent with the relatively small (20\%) permeability reduction.

Berea Core " $\mathrm{A}$ " $\left(\phi=19.50 \%\right.$ and $\left.\mathrm{K}_{\mathrm{o}}=13.20 \mathrm{md}\right)$ experienced a $72 \%$ permeability reduction $\left(\mathrm{K} / \mathrm{K}_{\mathrm{o}}=0.28\right.$; Figure 8$)$. Acetate levels were only measured during part of the experiment, but generally followed the same trend observed in the glass bead columns: a small but steady consumption within the core, yielding effluent concentrations $80 \%$ to $90 \%$ of the injected value. We attempted to image the growth using HRXCT and staining procedures analogous to those used in electron microscopy. The biofilm was perforated with a toluene solution in water to facilitate staining by $5 \mathrm{mM}$ uranyl acetate in deionized water. Uranyl ions are known to form complexes with nucleic acids. Cells would thereby absorb X-rays very effectively and thus exhibit sharp contrast with rock grains and pore space fluids. However, X-ray scans before and after the inoculation/growth phase did not show significant differences. We were thus unable to discern the mode of cell growth in the core.

A second column, Berea Core " $\mathrm{B}$ " where $\phi=19.20 \%$ and $\mathrm{K}_{\mathrm{o}}=12.30 \mathrm{md}$ was prepared, inoculated and run in a manner identical to the previous core. Permeability reduction was $45 \%\left(\mathrm{~K} / \mathrm{K}_{\mathrm{o}}=0.55\right.$; Figure 9$)$. In contrast to the other experiments, permeability declined steadily and relatively slowly for almost $50 \mathrm{PV}$ before reaching a constant value. Acetate consumption during the first $20 \mathrm{PV}$ was larger than at any time in any of the other experiments. Effluent acetate concentration then increased to values typical of the other experiments, i.e. $80 \%$ to $90 \%$ of the injected concentration.

Batch Experiments. Qualitatively similar cell growth was observed in all the batch experiments. After a lag period, there was an exponential growth phase and then a steady state phase (Figure 10). For samples $\mathrm{C} 3$ and $\mathrm{C} 4$, there was a high degree of clumping due to stirring. This caused the equipment to read an apparent decrease in the cfu count. The acetate history corresponded to the cell growth history: a lag phase, exponential decrease phase and finally a stabilization period. On average $100 \mathrm{ppm}$ of acetate remained in the flask at the end of each experiment (Figure 11). As described in the next section, the parameters in the Monod kinetics for the average behavior are found to be: maximum specific growth rate $m_{\max }=0.35 \mathrm{hr}^{-1}$, and half saturation constant $\mathrm{K}_{\mathrm{S}}=553 \mathrm{ppm}$. The stoichiometric coefficient $v$ was $4 \times 10^{9} \mathrm{cfu} / \mathrm{mg}$ acetate.

\section{Discussion}


Flow Experiments. The effluent reservoir in the flow-through system begins to show microbial colonization in each experiment after the permeability reductions have occurred. We infer that cells are detaching from the biofilm growing in the porous medium, being entrained in the fluid phase and swept out of the medium. Permeability reductions and acetate consumption did not change during this detachment phase. We interpret this as a steady state. Acetate is consumed at a small rate (catabolism) that does not lead to cell multiplication, and the small fluctuations in permeability correspond to shifts in the relative rates of detachment and straining of cells.

Overall permeability reduction varies significantly between replicate experiments. Acetate consumption is somewhat more consistent between replicates, but consumption does not always correlate with permeability reduction during an experiment. One reason for this variability is that cell growth does not appear to happen uniformly within a column or between replicates. Another may be that steady state is a delicate balance between growth and detachment. The coupling between nutrient consumption and cell growth may be strong at the pore scale. Preferential growth in one pore would divert flow of nutrients into to adjacent pores, but the local increase in flow rate would reduce the time available to microbes to consume those nutrients. This feedback loop could lead to scattered pockets of growth that result in variable macroscopic properties such as permeability reduction.

Images of growth vary from sample to sample. This may reflect the physical variability described above, but it may also be the result of differing staining methods. Figure 3 (bead pack 1 dyed red with TTC) indicates that the growth is discontinuous and sparse. This image indicates little to no permeability reduction would occur, but a decrease of almost 50\% was observed. On the other hand, Figure 6 shows that the growth was prevalent throughout a cross section of bead pack 3, located about $2 \mathrm{~cm}$ from the inlet face. This photograph is typical of a series of sections taken along a $2 \mathrm{~cm}$ length of the column. Growth in a region this large, coupled with the image (Figure 6), appears consistent with a $20 \%$ permeability reduction. Microbial plugging in this manner will yield a substantial permeability reduction if the microbes colonize a significant volume of the porous media.

Figure 7 shows high magnification of single beads (bead pack 3) with limited growth that resembles a grainy coating. This low-density growth would not cause significant permeability reduction. The beads shown in Figure 6 suggest that large permeability reductions will occur if the grainy growth expanded radially and concentrically coated grains encompassing pore bodies and pore throats similar to a calcitic or siliceous cement. Additional imaging will be conducted to determine whether this mode of growth occurs.

Batch Experiments. The Monod kinetics parameters $\mathrm{m}_{\max }$ and $\mathrm{K}_{\mathrm{S}}$ were extracted from the "average" acetate consumption and biofilm growth histories. The "average" curves are simply least-squares best fits to all four experiments plotted simultaneously (Figure 
10, Figure 11). To obtain the rate of biofilm generation $\mathrm{dB} / \mathrm{dt}$, the best-fit curve for biofilm growth was differentiated with respect to time. Applying Eq. 2 yields the specific growth rate as a function of time, from which the maximum value $\mathrm{m}_{\max }=0.35 \mathrm{~h}^{-1}$ is readily obtained. A plot of the specific growth rate $\mathrm{m}$ vs. acetate concentration (Figure 12) yields the half saturation constant $K_{S}$ : it is the acetate concentration at which the specific growth rate falls to half its maximum value. For these experiments, $\mathrm{K}_{\mathrm{S}}=553$ $\mathrm{ppm}$. A plot of biofilm growth rate $\mathrm{dB} / \mathrm{dt}$ vs. acetate consumption rate $-\mathrm{dA} / \mathrm{dt}$ was linear over most of the data range. The slope of that trend is related by Eq. 4 to the stoichiometric coefficient, found to be $v=4 \times 10^{9} \mathrm{cfu} / \mathrm{mg}$ acetate.

The Monod curve (Figure 12) does not match the form of classic Monod curves. It may be that other nutrients besides acetate are affecting the growth rate. Nevertheless, the biofilm growth predicted with Eqs. $1-4$ and the values of $\mathrm{m}_{\max }, \mathrm{K}_{\mathrm{S}}$ and $v$ agree reasonably well with the best-fit data (Figure 13). The acetate consumption model was also calculated using the stoichiometric coefficient and the calculated values were similar to the values measured by the best-fit curve (Figure 14).

The amount of acetate remaining at the end of the biofilm exponential growth phase in the batch experiments suggests that acetate is not the limiting substrate in the experiment. This raises the possibility of surface area of the biofilm limiting the growth.

\section{Conclusions}

1. Both glass bead columns and Berea cores inoculated with a model microbe ( $P$. aeruginosa PAO-1) exhibited appreciable permeability reductions (20\% to $70 \%)$ during continuous injection of nutrients. Interestingly, the reductions reach a plateau value, usually within a few PV, although in one case it required nearly 50 PV to establish the plateau. The physical reason for the plateau remains to be determined, but it may be a consequence of the coupling between nutrient supply, biofilm growth and permeability reduction at the pore-scale.

2. The trend of permeability reduction and acetate utilization with time varied between replicate experiments. Biostaining at the end of replicates also showed qualitative differences in locations of microbial growth. These factors make development of a quantitative model difficult. More effective biostaining must be utilized to better characterize microbial colonization in porous medium.

3. Using the "average" behavior observed in batch experiments to obtain kinetic parameters, the Monod equations adequately describe the trend of cell growth and acetate consumption. The amount of acetate left at the end of the experiments was enough for growth to continue. This raises the possibility that other factor such as surface area limiting the biofilm growth. The batch data exhibited considerable scatter between each experiment. 
Topic 1: Experimental evaluation of growth

\section{References}

Cunningham, A.B. et al.: "Influence of Biofilm Accumulation on Porous Media Hydrodynamics," Environmental Science Technology, Vol 25 \#, 1991.

Dunsmore, B.C. et al.: "A Novel Approach to Investigate Biofilm Accumulation and Bacterial Transport in Porous Matrices," Environmental Microbiology 2004 6(2), 183-187.

Dworkin, M. et al.: "Experiments With Some Microorganisms Which Utilize Ethane and Hydrogen," Journal of Bacteriology, (May 1958). 75(5): 592-603

Lee, H.O.et al.: "Effects of Variables on Microbial Profile Modification with Spores," SPE 39671 (1998).

Rittman, B.E. et al.: "Evaluation of Steady State Biofilm Kinetics," Biotech. Bioeng. (1980), 2343-2357.

Vadie, A. J. et al.: "Utilization of indigenous microflora in permeability profile modification of oil bearing formations," SPE 35448 presented at SPE/DOE 10 ${ }^{\text {th }}$ Improved Oil Recovery Symp., Tulsa, Oklahoma, 21-24 April, 1996.; SPERE (Feb 2002) 33-41

Vandevivere, P. et al.: "Relationship between Transport of Bacteria and Their Clogging Efficiency in Sand Columns,"Applied and Environmental Microbiology (August, 1992) 2523-2530.

Williamson, K.J. et al.: "A Model of Substrate Utilization by Bacterial Films," J. Water Pollution Control Fed. (1976), 48:9-24. 
Topic 1: Experimental evaluation of growth

Table 1. Flow experiment results.

\begin{tabular}{|l|c|c|c|c|c|}
\hline $\begin{array}{c}\text { Porous } \\
\text { media }\end{array}$ & $\Phi$ & $\mathrm{K}_{\mathrm{O}} \mathrm{md}$ & $\mathrm{K}$ md & $\mathrm{K} / \mathrm{K}_{\mathrm{O}}$ & $\begin{array}{c}\text { Time to first } \\
\text { plateau in } \\
\text { permeability } \\
\text { reduction }\end{array}$ \\
\hline Bead pack \#1 & $39.40 \%$ & 1483.0 & 830.5 & 0.56 & $3 \mathrm{PV}$ \\
\hline Bead pack \#2 & $38.80 \%$ & 1414.0 & 650.4 & 0.46 & $12.5 \mathrm{PV}$ \\
\hline Bead pack \#3 & $38.55 \%$ & 1375.0 & 1100.0 & 0.80 & $3.5 \mathrm{PV}$ \\
\hline Core A & $19.50 \%$ & 13.2 & 3.7 & 0.28 & $22 \mathrm{PV}$ \\
\hline Core B & $19.20 \%$ & 12.3 & 6.8 & 0.55 & $51 \mathrm{PV}$ \\
\hline
\end{tabular}




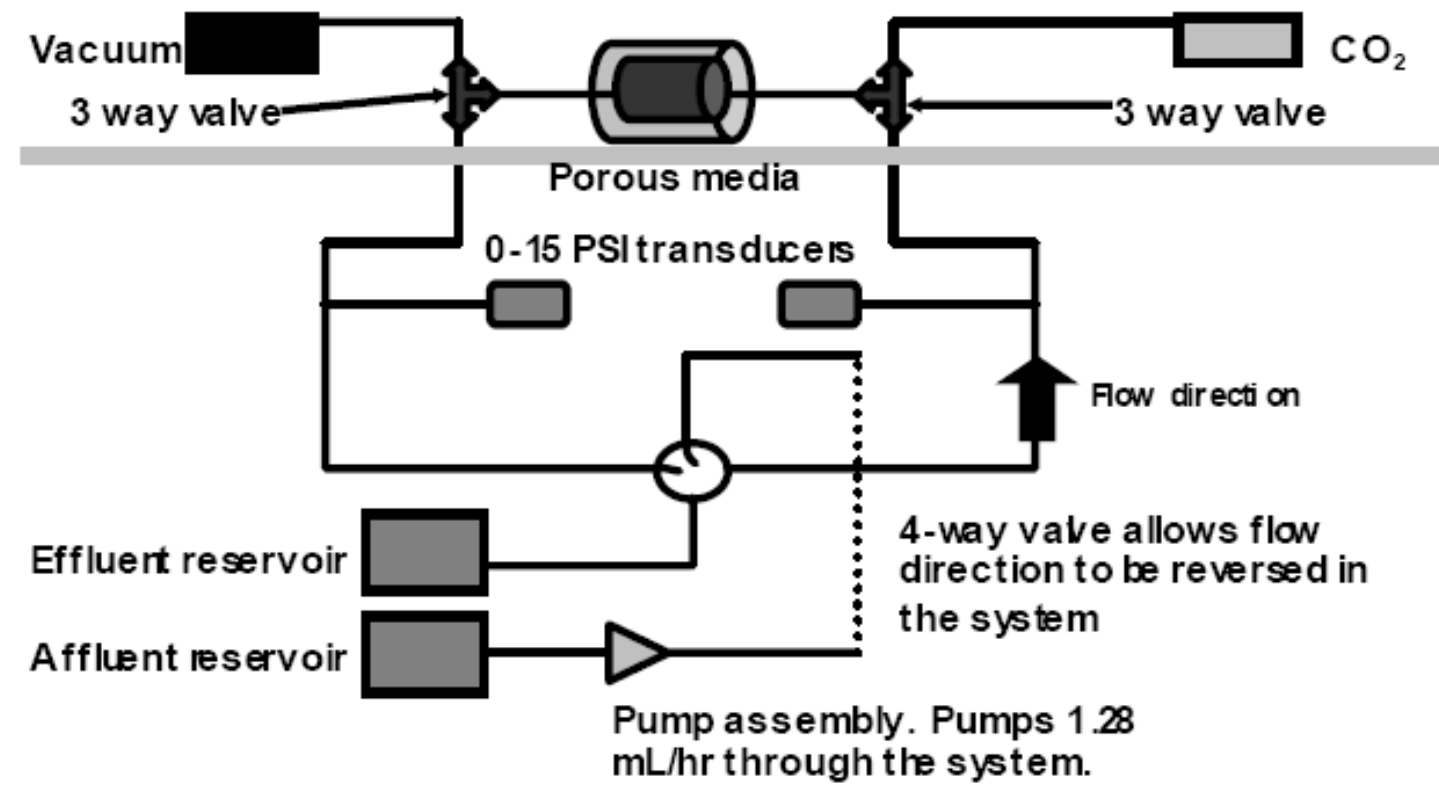

Figure 1. Flow through system for in-situ growth of microbes within a core. Objects below grey line are maintained at $44^{\circ} \mathrm{C}$, so that growth occurs only in the porous medium. 


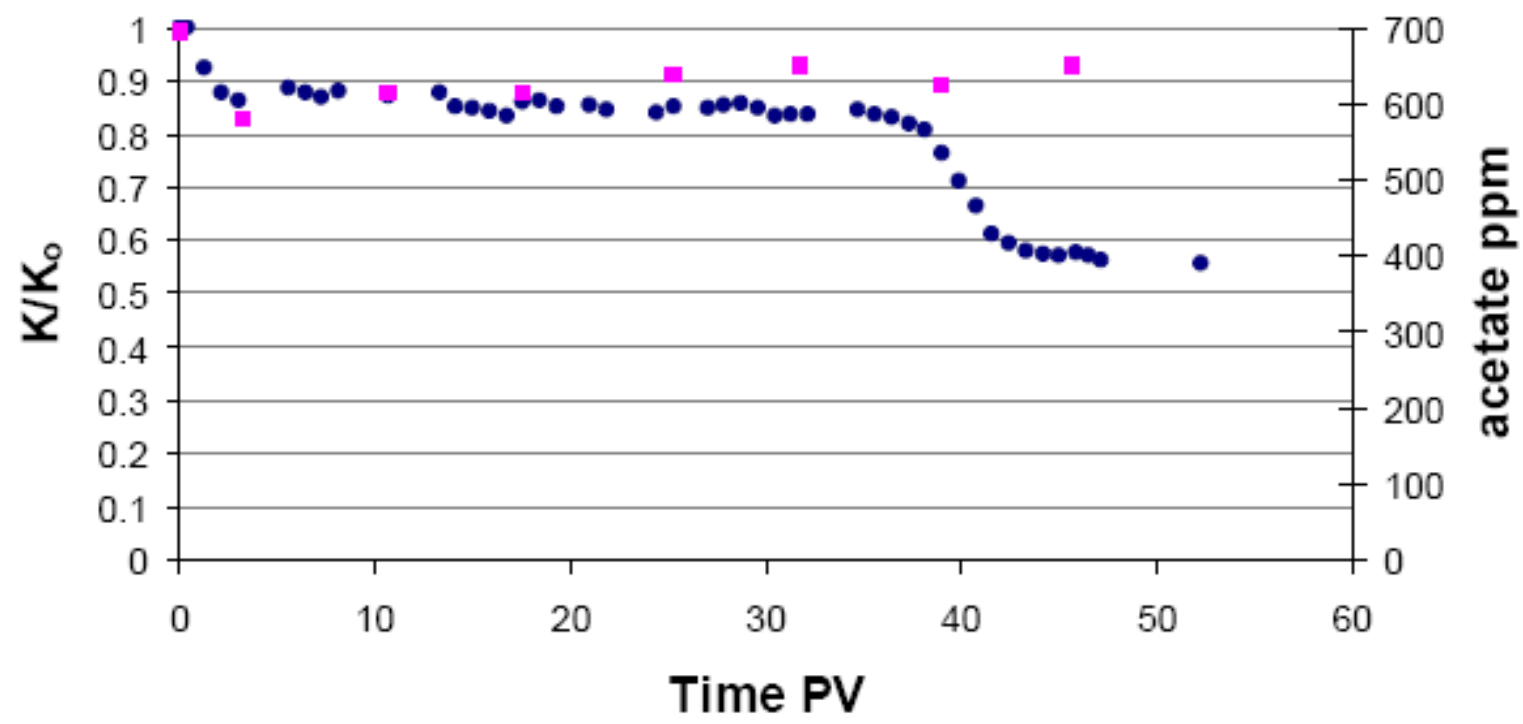

- permeability - acetate

Figure 2. Permeability reduction and effluent acetate concentrations (injected value $700 \mathrm{ppm}$ ) for bead pack \#1. 
Topic 1: Experimental evaluation of growth

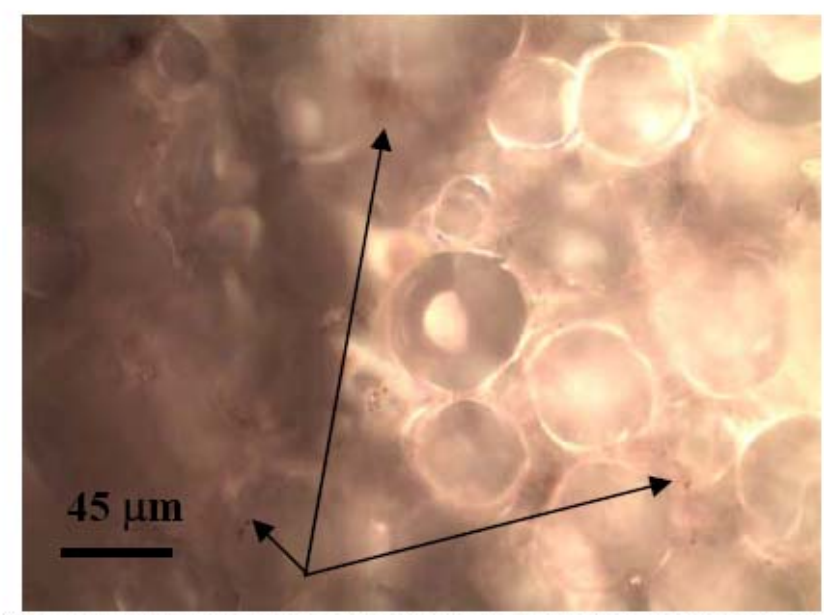

Figure 3. Pseudomonas Aeruginosa colonies stained red with TTC, after polymer has set within Bead pack \#1. Red tint indicates TTC reduction by microbial colonies.

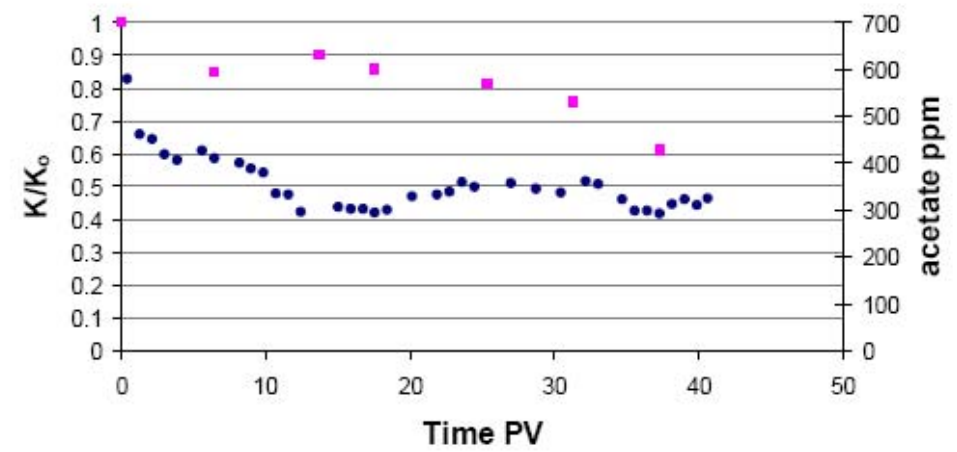

- permeability = acetate

Figure 4. Permeability reduction and effluent acetate concentrations (injected value $700 \mathrm{ppm}$ ) for bead pack \#2.



- permeability $\approx$ acetate

Figure 5. Permeability reduction and effluent acetate concentrations (injected value $700 \mathrm{ppm}$ ) for bead pack $\# 3$. 
Topic 1: Experimental evaluation of growth

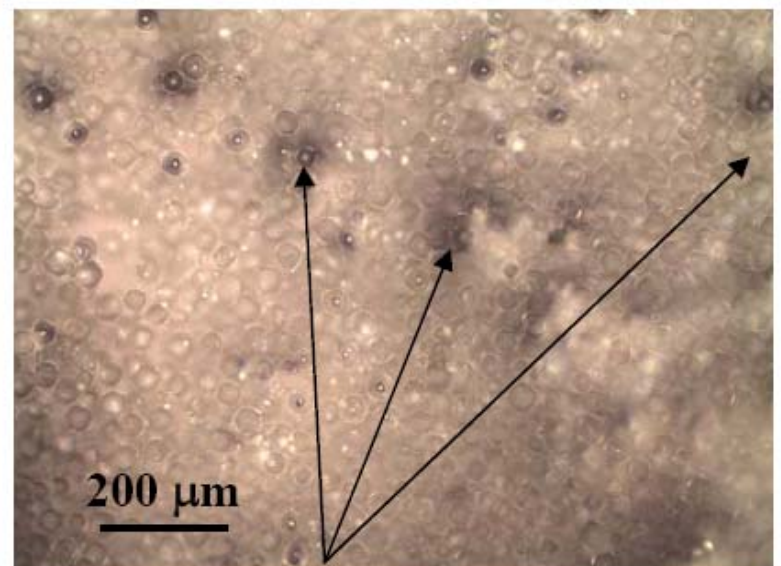

Figure 6. Bead pack \#3 stained with NBT solution. Dark patches are microbial colonization

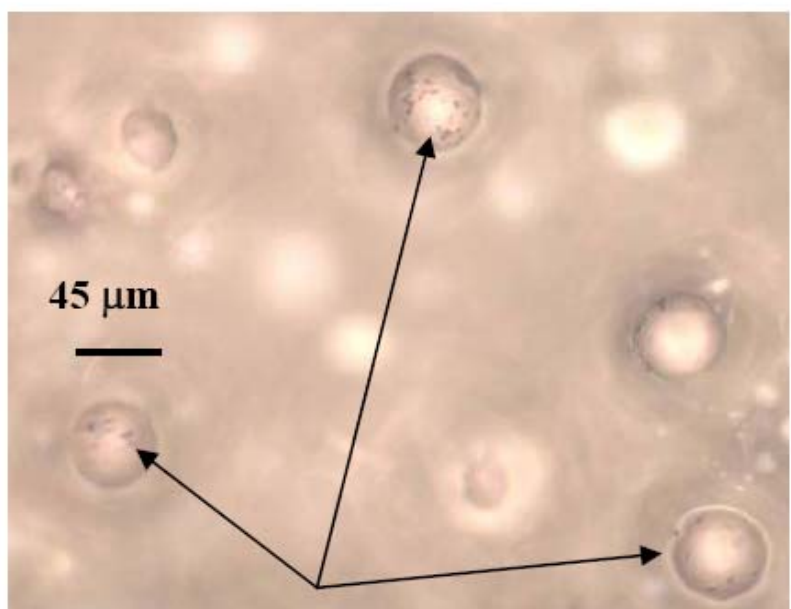

Figure 7. Grain coating microorganisms stained with NBT in Bead pack \#3 at high resolution.

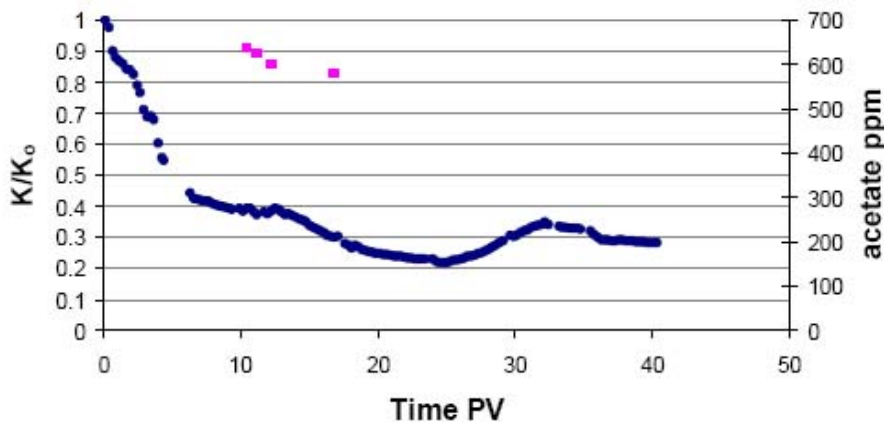

- permeability " acetate

Figure 8. Permeability reduction and effluent acetate concentrations (injected value $700 \mathrm{ppm}$ ) for core $A$. 
Topic 1: Experimental evaluation of growth

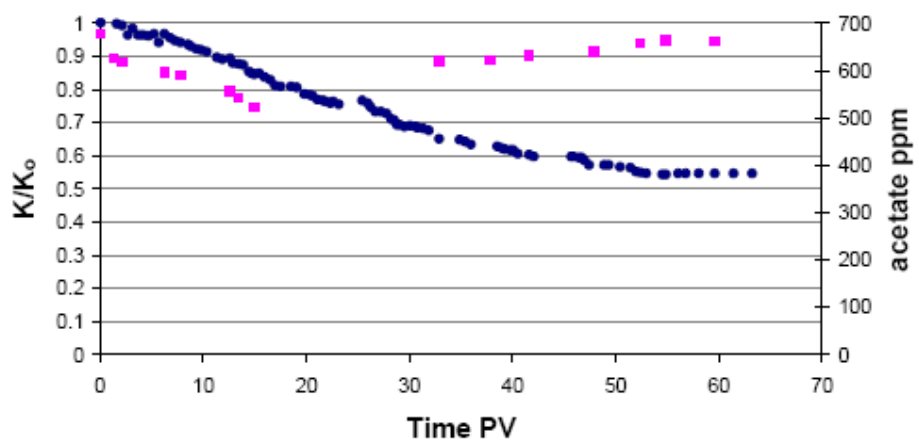

- permeability = acetate

Figure 9. Permeability reduction and effluent acetate concentrations (injected value $700 \mathrm{ppm}$ ) for core B.

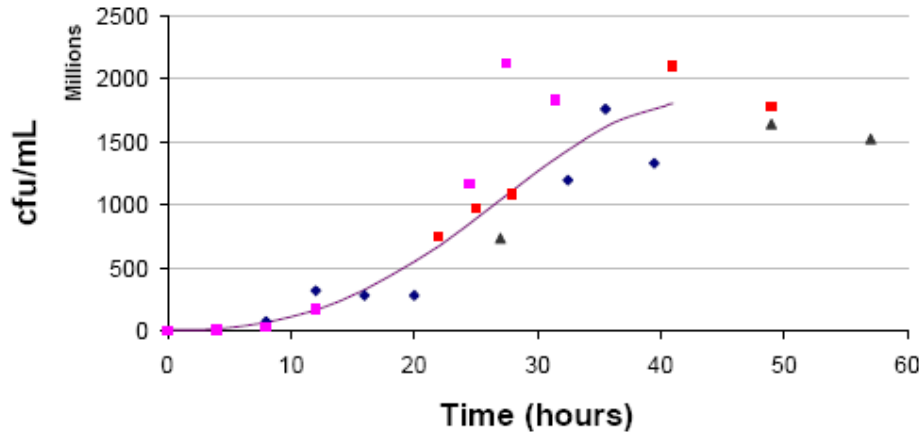

- C1 $\| \mathrm{C} 2 \star \mathrm{C} 3$ - C4 - Best-fit Line

Figure 10. Biofilm growth with time for replicate batch experiments

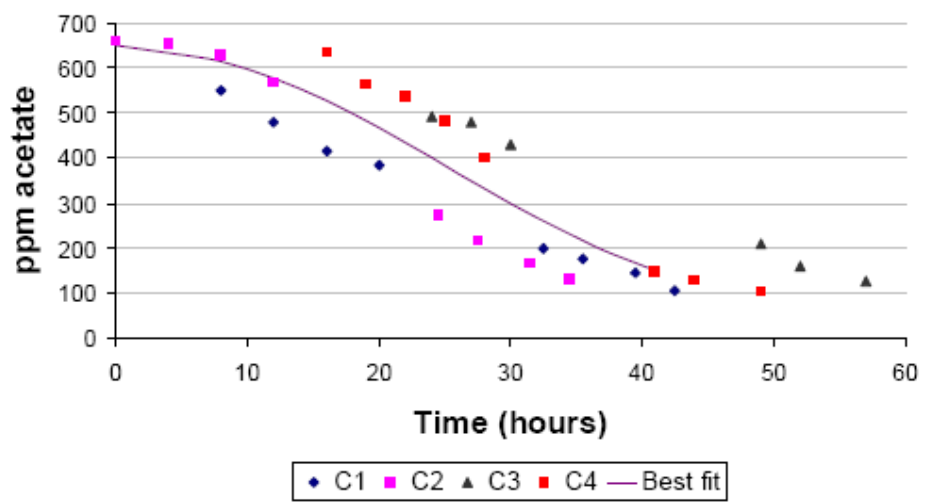

Figure 11. Acetate consumption with time in batch growth experiments. 
Topic 1: Experimental evaluation of growth



Figure 12. Monod Growth Curve for best fit growth data

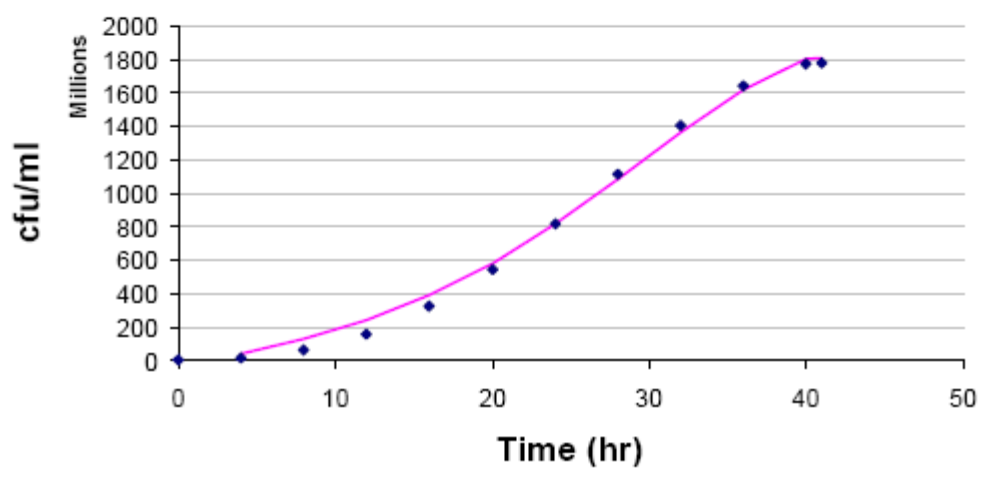

- Measured Biofilm - Calculated Biofilm

Figure 13. Comparison of calculated and observed best fit biofilm growth.

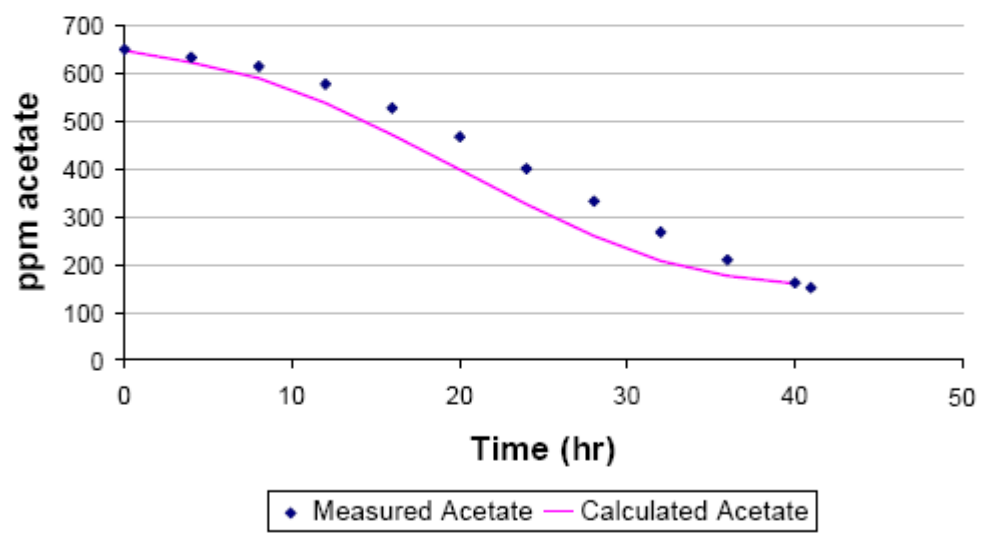

Figure 14. Comparison of calculated and observed best fit acetate consumption. 


\section{Topic 1: COMPLETE CATALOG OF EXPERIMENTS -- Apparatus Flow through System}

The flow-through system (Figure 3.1) was built specifically to supply very low flow rates. With low flow rates, small diameter porous rock or glass bead columns can be utilized to simulate field-scale waterflood velocities.

In Table 3.1, interstitial velocity $u$ is calculated as $(24 q) /(A \phi)$

Table 3.1. Flow rates and pore velocities in porous media samples.

\begin{tabular}{|l|c|c|c|c|c|}
\cline { 2 - 6 } \multicolumn{1}{c|}{} & $\mathrm{q} \mathrm{cm}^{3} / \mathrm{hr}$ & $\mathrm{A} \mathrm{cm}^{2}$ & $\phi$ & $\mathrm{u} \mathrm{cm} /$ day & $\mathrm{u} \mathrm{ft} /$ day \\
\hline Berea core & 1.28 & 5.07 & 0.19 & 31.91 & 1.05 \\
\hline Bead pack & 1.28 & 0.79 & 0.38 & 102.93 & 3.38 \\
\hline
\end{tabular}

The pump is a FMC 6 RPM piston pump that can pump 0 to $20 \mathrm{~mL}$ per hour. All system tubing is $1 / 8$ " OD PTFE Teflon. Small diameter tubing was needed to keep the volume in the flow lines small compared with the pore volume within the samples. Teflon materials were selected because it provides an inert surface and reduced adherence by microbial cells. System plumbing and connections are made exclusively of Swagelok fittings composed of inert stainless steel and Teflon. The total system volume with flow lines, connecting pieces, pressure transducers including the core itself is $16 \mathrm{~mL}$. 


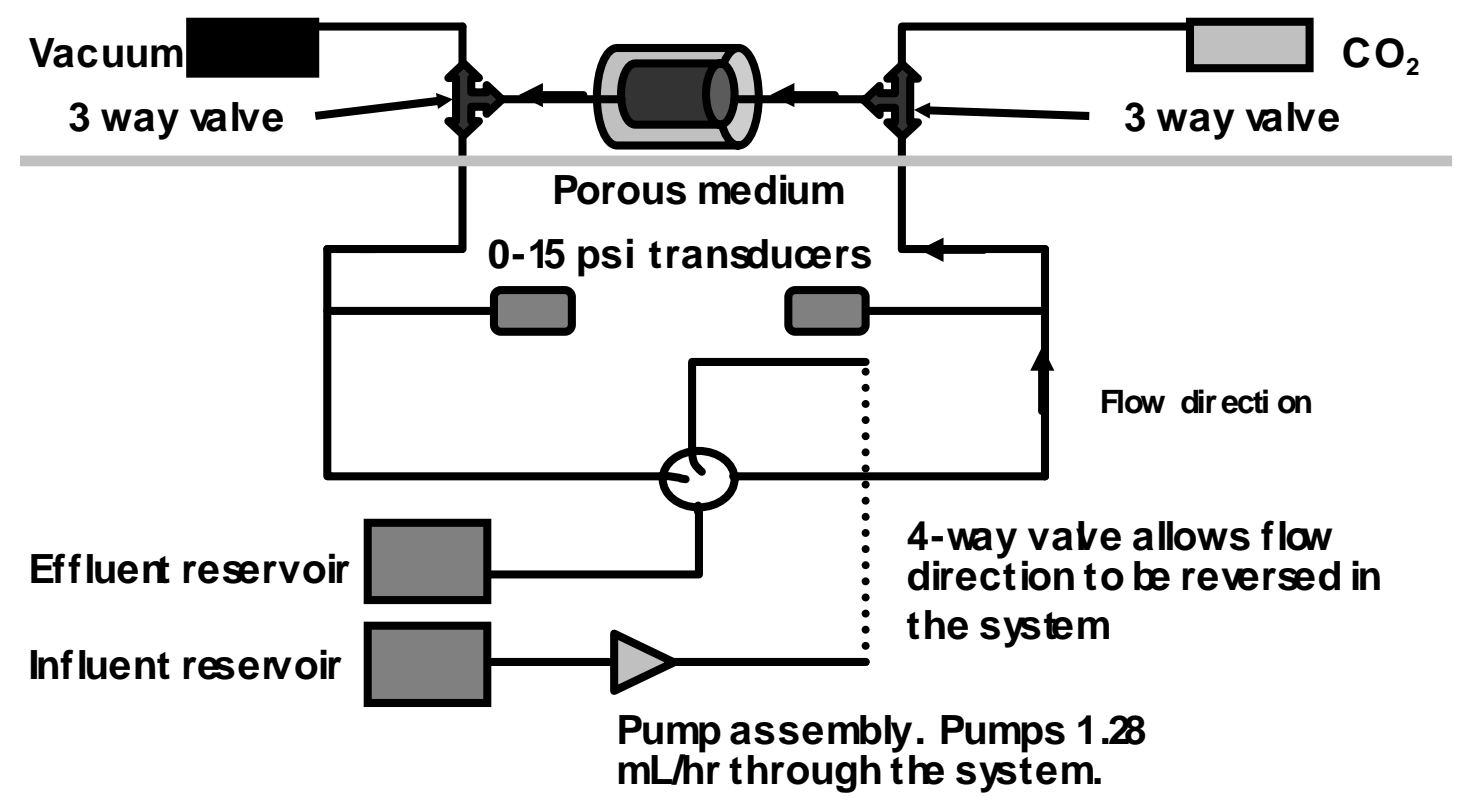

Figure 3.1: Flow-through system. Objects below gray line are maintained in oven at $45^{\circ} \mathrm{C}$. Objects above are maintained at room temperature.

\section{Pressure Measurement}

Validyne P2 (0-15 psi) pressure transducers were used for monitoring pressure within the system. Transducers are placed on both the influent and effluent ends of the core. Each transducer measures absolute pressure, so the pressure drop across the core is simply the difference between the two transducer readings. The low-pressure transducers are suitable for these low flow rate experiments, and give accuracy to 0.0015 psi. The pressure transducers output voltage signal was routed to a National Instruments (NI) data acquisition board, desktop computer and NI Datalogger software enabling continuous pressure monitoring at 9-second intervals.

\section{Temperature modification of system}

Pseudomonas aeruginosa is known to grow well at room temperature, but not when ambient temperature exceeds $45^{\circ} \mathrm{C}$. This was verified in the lab prior to the experiments by placing viable cells on an agar culture plate in a $45^{\circ} \mathrm{C}$ incubator for a 24 -hour period. We observed no microbial growth on the plate during this time. The plate was then removed from the oven and incubated at room temperature for 24 hours. Colony 
formation was the same as for control plates that were not exposed to the higher $45^{\circ} \mathrm{C}$ thus indicating that $45^{\circ} \mathrm{C}$ prevents growth (i.e., bacteriostatic) but does not kill the cells. Therefore the core was placed at $24^{\circ} \mathrm{C}$ to promote growth within the core, and the rest of the plumbing, transducers and pump head were placed at $45^{\circ} \mathrm{C}$ to inhibit growth but not to kill the cells. Figure 3.2 is a photograph of the apparatus showing all of the plumbing, transducers and pump in the $45^{\circ} \mathrm{C}$ incubator and the column on top of the incubator at $24^{\circ} \mathrm{C}$. Although the pump was in the incubator, the pump motor was encased in a foam housing and ducted with room temperature air. These precautions were needed to prevent thermal failure of the pump motor and also to prevent spiking of incubator temperatures above $45^{\circ} \mathrm{C}$ due to the hot electric motor.

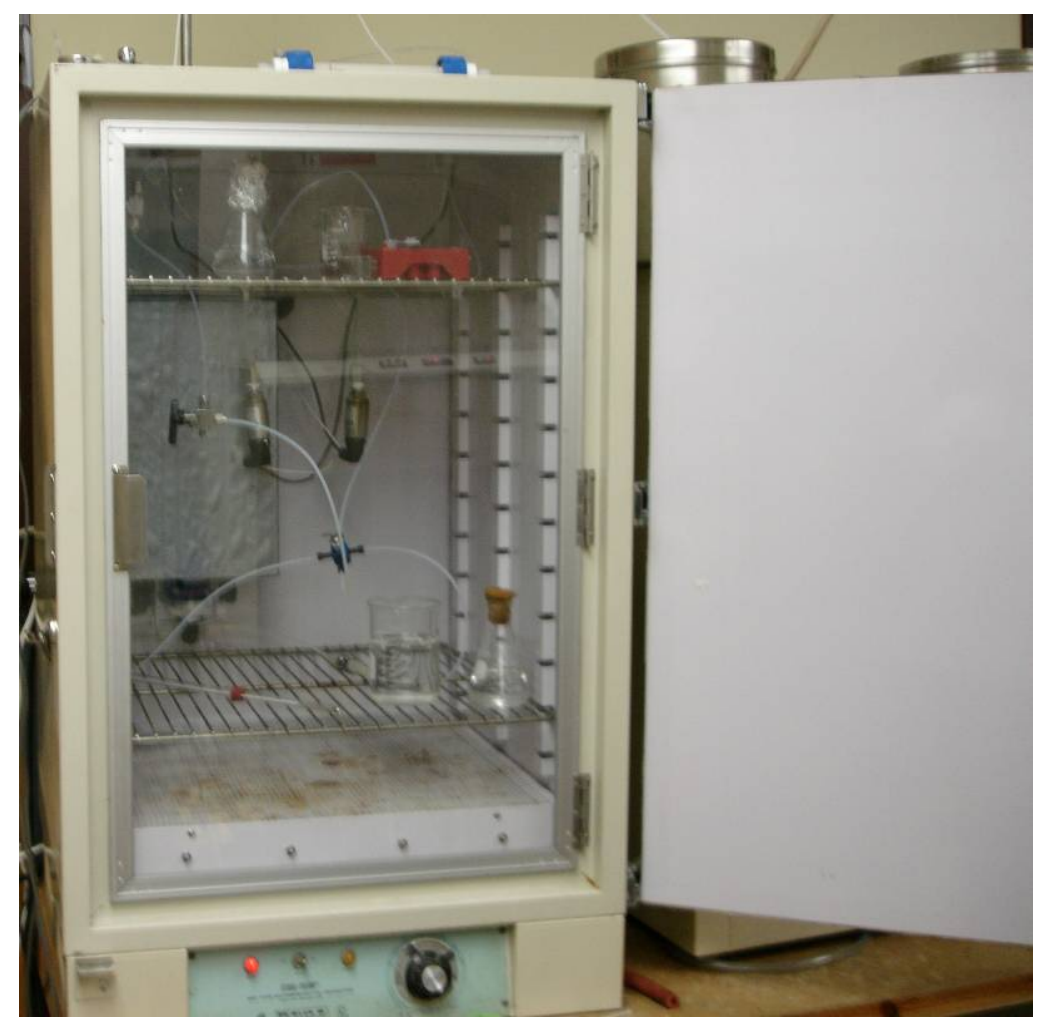

Figure 3.2: Flow-through system maintained in incubator with porous column on top. 


\section{Bead pack description}

Lead-free silica glass beads were selected to make an inert bead pack with very uniform porosity. The beads were sieved between 325 and 400 mesh screens to yield a bead size of 38-43 $\mu \mathrm{m}$ diameters. These were packed in a $15 \mathrm{~cm}$ long by $1 \mathrm{~cm}$ ID Kontes brand, glass chromatography column (Figure 3.3) which proved to have the requisite dimensions to give a measurable pressure drop across the high permeability $(1500 \mathrm{md})$ bead pack.

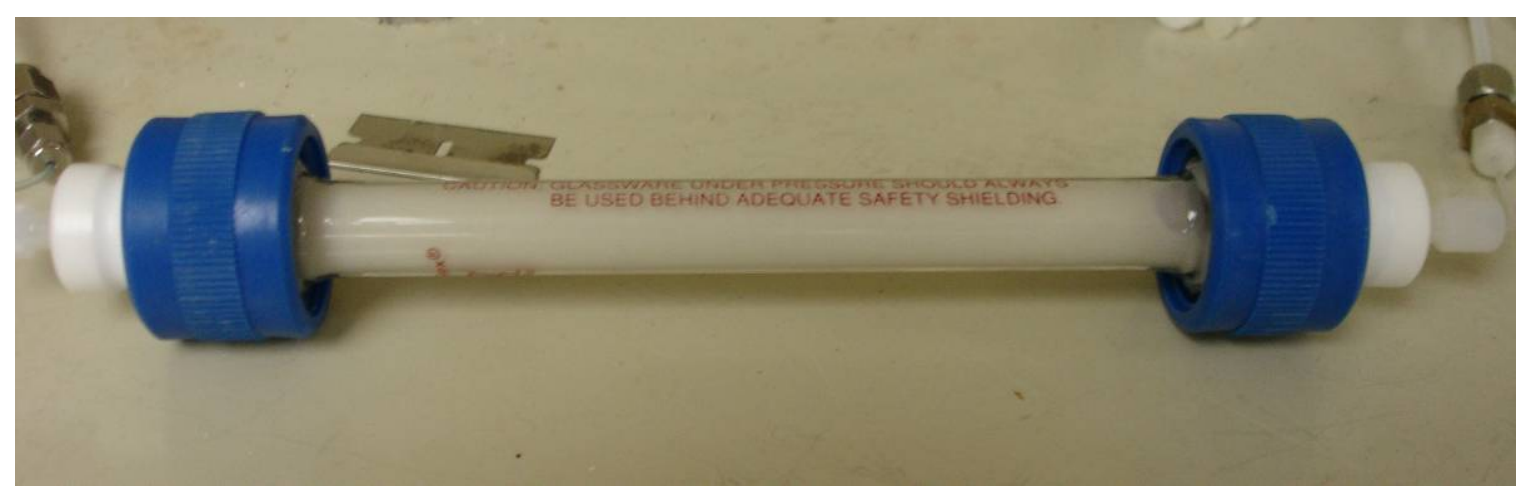

Figure 3.3: Bead pack in chromatography tube.

\section{Berea description}

Berea cores 1" diameter and 2" long were cut from a single, uniform block and fashioned into columns. The cores have approximately $19 \%$ porosity and $50 \mathrm{md}$ permeability. The Berea sandstone is composed mostly of quartz $(\sim 90 \%)$ and includes small percentages of feldspar and iron clays. The cores were capped with Lexan (polycarbonate) plastic end caps with a 1/16" recessed headspace in order to allow fluid to contact the maximum amount of core surface (Figure 3.4). After the end caps were secured with epoxy to the core, the entire piece was potted in epoxy so that the core was confined by approximately 0.25 in. thick sidewalls (Figure 3.5). The epoxy sets very rapidly, ensuring that the penetration of epoxy into the core is minimal. This proved to be a very effective core holding apparatus. The outer side of the end caps were tapped to allow for Swagelok 1/8" unions to be screwed into the end caps. The unions were wrapped in Teflon tape to 
Topic 1: Experimental evaluation of growth

prevent leakage. The swage unions again were Teflon because it is inert and should reduce adhesion of microbes.

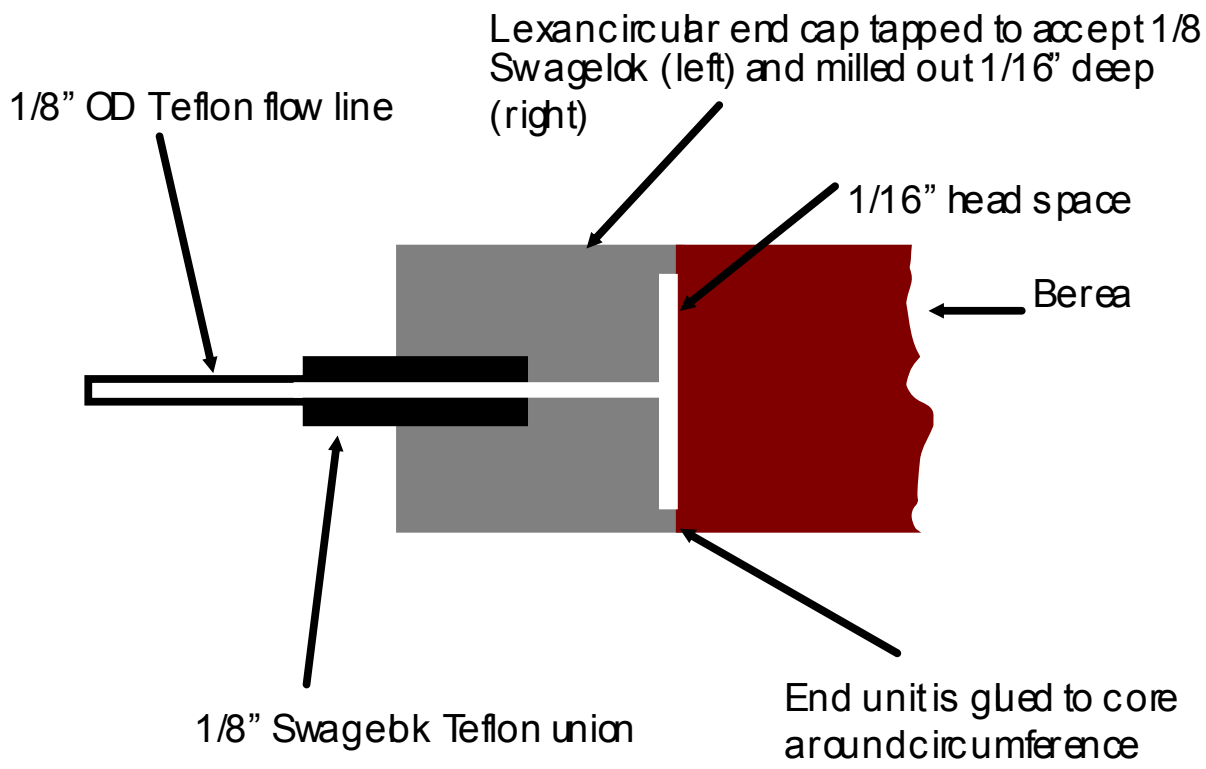

Figure 3.4: Berea core end unit sketch from side view (not to scale). Note core end unit (grey box) and core (brown) are circular. 


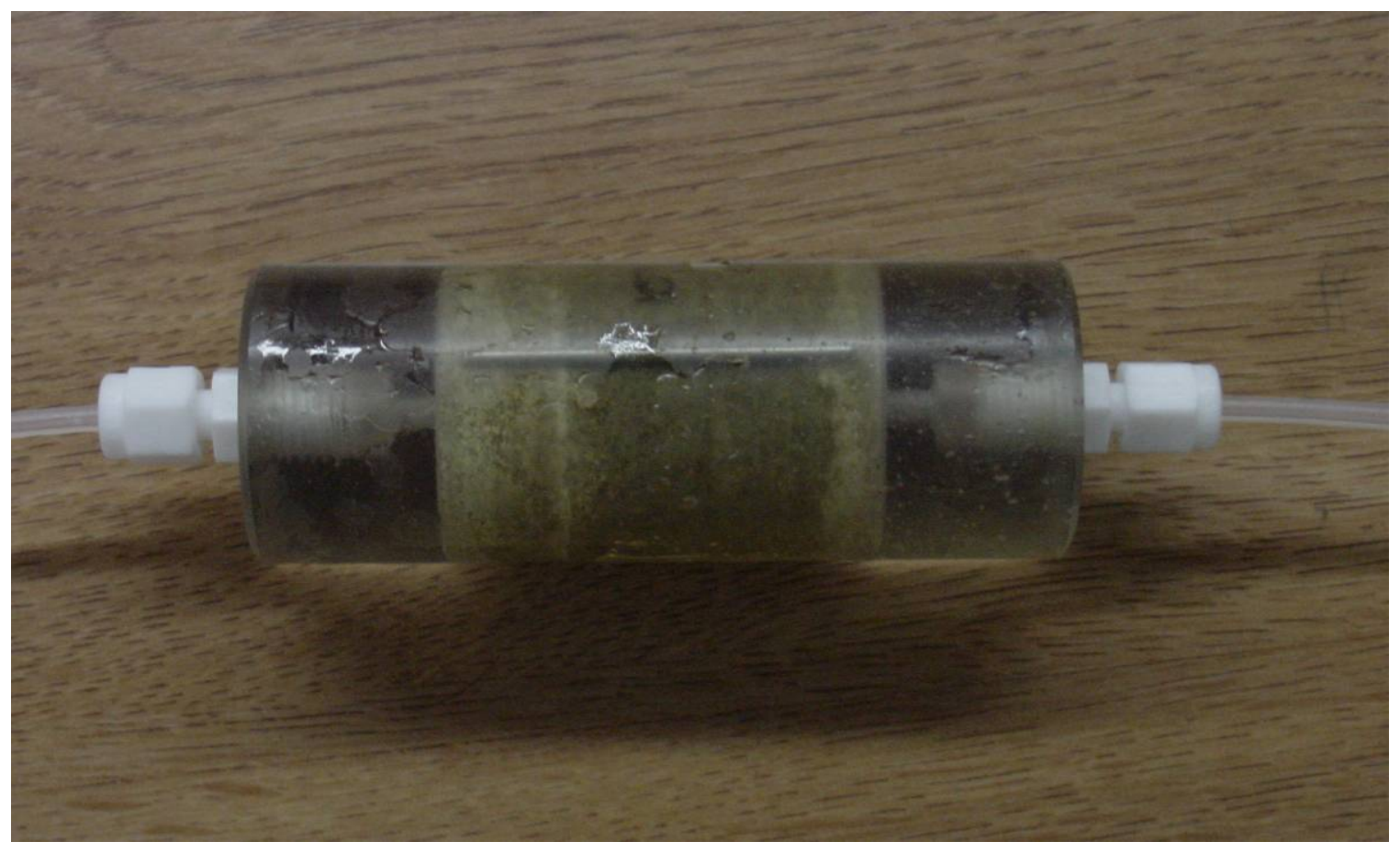

Figure 3.5: Berea core in epoxy coating with Lexan end caps providing 1/16” headspace at core entrance and exit. End caps are tapped to accept 1/8” Swagelok ${ }^{\circledR}$ fittings.

\section{Core flooding procedures and preparatory work}

Core flooding procedures were followed to ensure that cores were fully saturated with fluids and did not contain air in small pores. Since air is not water-soluble compared with other gasses, the air must be completely removed from the core and exchanged with a water-soluble gas such as $\mathrm{CO}_{2}$. The core was evacuated first in a vacuum for 2-3 hours; $\mathrm{CO}_{2}$ was introduced via a 3-way valve until pressure reached $20 \mathrm{psi}$. The core apparatus was then immersed in water to check for leaks. The position of the 3-way valve was changed to re-establish a vacuum, followed by re-pressurization with $\mathrm{CO}_{2}$. The vacuum and $\mathrm{CO}_{2}$ flushing process is repeated 3-4 times to make sure that any gas remaining in the core will be the highly water-soluble $\mathrm{CO}_{2}$ and not air. After the final $\mathrm{CO}_{2}$ flush and under vacuum the core is injected with aerated Dworkin Foster mineral medium (Dworkin and Foster, 1958) described in later in this chapter. 


\section{Core inoculation}

The core was inoculated with a young, rapidly growing culture of $P$. aeruginosa in Dworkin Foster medium with $0.1 \%$ sodium acetate as carbon and energy source. The culture was diluted to $\sim 10^{7} \mathrm{cfu} / \mathrm{mL}$ (cfu = colony forming units, or "cells") for injection in order to avoid face plugging by higher cell concentrations (see Lee and Bae, 1998). A total of $7 \mathrm{PV}$ of inoculant (equal to $3 \times 10^{8} \mathrm{cfu}$ ) was injected at $20 \mathrm{~mL} / \mathrm{hr}$ into the core during a 1.5-hour period. It is not known how many of the inoculated cells are retained in the cores. After inoculation the pumping of growth medium was returned to $1.28 \mathrm{~mL} / \mathrm{hr}$, and pressure was continually monitored across the column as an indicator of biofilm formation. Effluent sodium acetate concentration was monitored via gas chromatography every 2-3 PV. The acetate utilization and increased pressure differential were indicators of biofilm growth.

\section{System Sterility}

Aseptic techniques were used to ensure that Pseudomonas aeruginosa was the only culture in the core system. After the system was plumbed and assembled, a 70\% isopropanol solution was pumped through the entire system followed by sterile water and finally sterile growth medium. Fluids were sterilized by autoclaving, and a laminar flow hood was used to prepare solutions for pumping.

\section{Selection of Mineral Growth Medium}

Both Dworkin Foster and M9 (Short Protocols in Molecular Biology, 2002) growth media were tested for use in core column experiments. Growth of $P$. aeruginosa in M9 medium had more pronounced clumping and evidence of cell lysis in liquid cultures when maximal growth was achieved. There was a concern that M9 medium would not be optimal for biofilm growth in the cores. Therefore, Dworkin Foster medium was selected for continued experiments because the growth in liquid culture was abundant and showed no signs of lysis. The composition of both Dworkin Foster and M9 media are given below. 
Table 3.2: M9 mineral medium composition

\begin{tabular}{|c|c|}
\hline $6 \mathrm{~g} / \mathrm{L}$ & $\mathrm{Na}_{2} \mathrm{HPO}_{4}$ \\
\hline $3 \mathrm{~g} / \mathrm{L}$ & $\mathrm{KH}_{2} \mathrm{PO}_{4}$ \\
\hline $1 \mathrm{~g} / \mathrm{L}$ & $\mathrm{NH}_{4} \mathrm{Cl}$ \\
\hline $0.015 \mathrm{~g} / \mathrm{L}$ & $\mathrm{CaCl}_{2}$ \\
\hline $0.12 \mathrm{~g} / \mathrm{L}$ & $\mathrm{MgSO}_{4} 7 \mathrm{H}_{2} \mathrm{O}$ \\
\hline
\end{tabular}

Table 3.3: Dworkin Foster mineral medium composition

\begin{tabular}{|c|c|}
\hline $6 \mathrm{~g} / \mathrm{L}$ & $\mathrm{Na}_{2} \mathrm{HPO}_{4}$ \\
\hline $4 \mathrm{~g} / \mathrm{L}$ & $\mathrm{KH}_{2} \mathrm{PO}_{4}$ \\
\hline $2 \mathrm{~g} / \mathrm{L}$ & $\left(\mathrm{NH}_{4}\right)_{2} \mathrm{SO}_{4}$ \\
\hline $0.4 \mathrm{~g} / \mathrm{L}$ & $\mathrm{NaCl}$ \\
\hline $0.2 \mathrm{~g} / \mathrm{L}$ & $\mathrm{MgSO}_{4} 7 \mathrm{H}_{2} \mathrm{O}$ \\
\hline $0.001 \mathrm{~g} / \mathrm{L}$ & $\mathrm{FeSO}_{4} 7 \mathrm{H}_{2} \mathrm{O}$ \\
\hline
\end{tabular}

The carbon and energy source was acetate which does not produce fast growth nor high cell numbers in comparison to substrates such as glucose. However, the acetate was easily quantified by gas/liquid chromatography and it more closely represents available carbon sources in the subsurface.

\section{Batch Growth Experiments}

Batch growth experiments were performed to provide growth data that could be used to interpret results from experiments on biofilm growth in cores and beads. Pseudomonas aeruginosa PAO1 was selected as the only test organism for several reasons. First, P. aeruginosa has been widely studied as an aerobic biofilm-forming microbe because of its association with cystic fibrosis patients who often experience lung infections by this organism. Second, it can be grown as a biofilm in defined mineral media using a simple carbon source such as acetate. Third, it grows readily at ambient laboratory temperature $\left(\sim 24^{\circ} \mathrm{C}\right)$ but not at temperatures above $\sim 44^{\circ} \mathrm{C}$ which affords a mechanism to limit growth to the core or bead column (Figure 3.1). Lastly, P. aeruginosa is typically a resident of 
Topic 1: Experimental evaluation of growth

soils and water and thus is a reasonable model organism for subsurface flora, even though this microorganism is not considered as indigenous to oil reservoirs.

The use of a single culture raises the issue of whether the biofilm growth by $P$. aeruginosa represents the biofilm by indigenous reservoir microbes. The answer to this concern is simply that there is scant information on the typical flora of oil reservoirs, and it is probable that the mode of growth differs according to temperature and geochemistry. The advantage of a single culture is that it can be used to understand the basic principles of microbial plugging (i.e. the relationship between nutrient uptake, microbe multiplication, and permeability reduction) without having to distinguish the contributions of each microbial consortium member in a biofilm composed of numerous microbial species.

Sodium acetate utilization was monitored by gas/liquid chromatography. Culture samples were filtered through a $0.45 \mu \mathrm{m}$ PVDF or nylon syringe filter and acidified with dilute $\mathrm{HCl}$. Chromatographic conditions were as follows: $0.4 \mu 1$ sample; $0.53 \mathrm{~mm} \times 30 \mathrm{~m}$ Alltech ATwax capillary column; $120^{\circ} \mathrm{C}$ isothermal; FID. Concentrations were calculated using a standard curve ranging from 0 to $1000 \mathrm{ppm}$ acetate (Figure 3.6). 


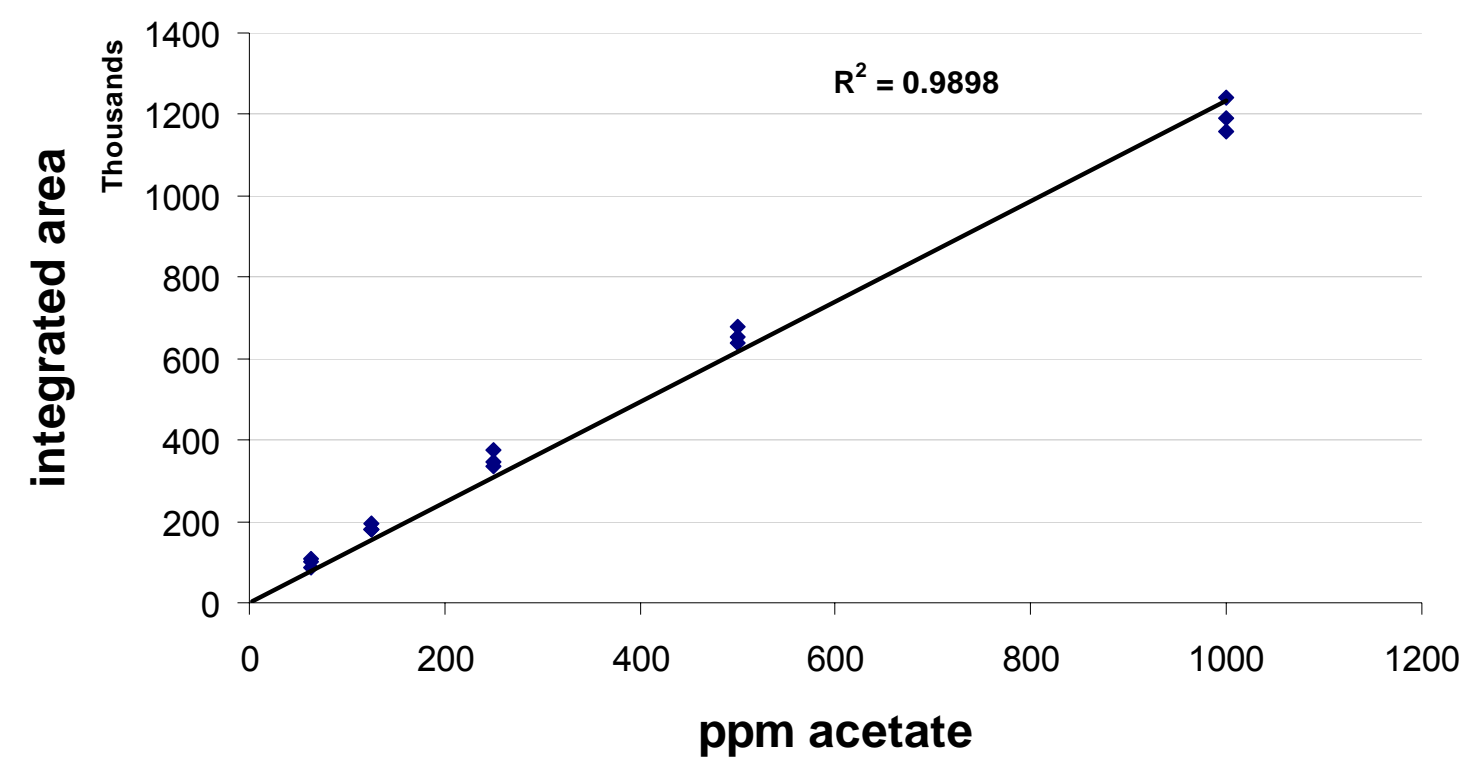

Figure 3.6: Example of Acetate Standard Calibration Plot

The batch growth of $P$. aeruginosa PAO1 in Dworkin Foster mineral medium with $0.1 \%$ sodium acetate is shown in Figures 3.7-3.10. These aerobic growth experiments were performed with growth medium in $250 \mathrm{~mL}$ Erlenmeyer flasks at room temperature (22 C) without shaking. Typical shaking of the culture produced excessive clumping of cells which interfered with plate count enumeration. The plot of cfu $/ \mathrm{mL}$ vs. time shows scatter in the points because the enumeration of viable cells using plate count methods normally has a wide deviation of values. The static cultures still produced cell aggregation rather than uniform unicellular suspensions, and this resulted in different plate count data during the exponential and stationary phases of growth. 


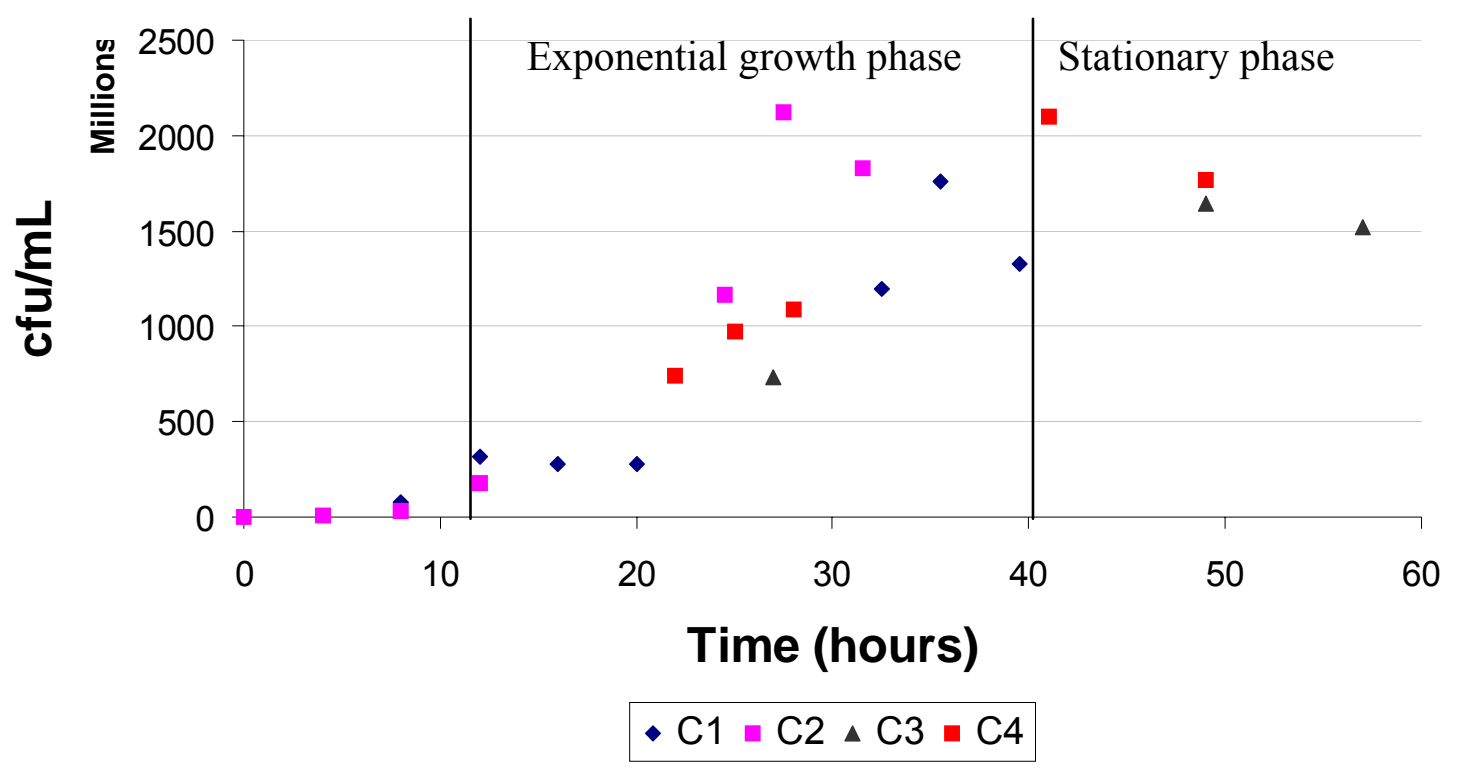

Figure 3.7: Batch experiment plate count data for four separate cultures (C1-C4); initial acetate concentration of $700 \mathrm{ppm}$.

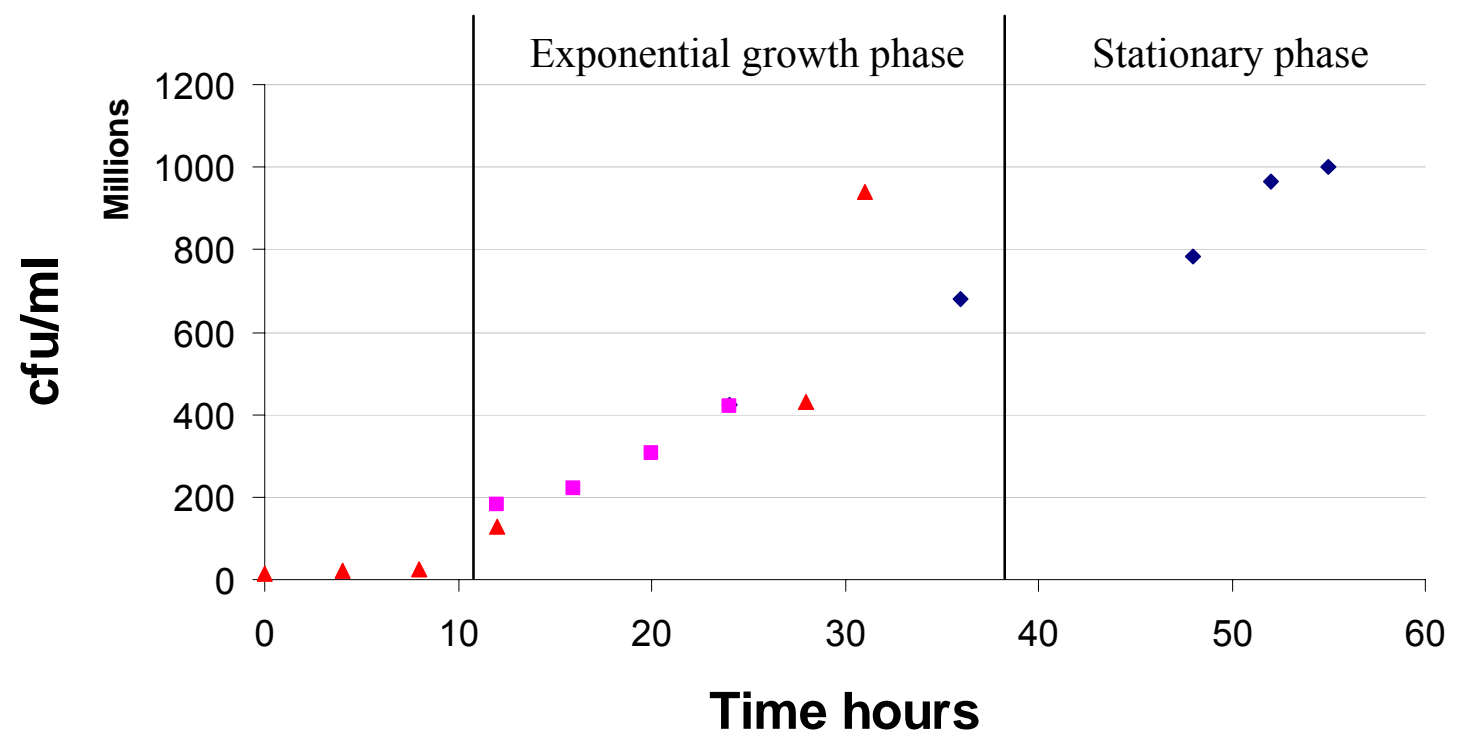

- C1 - C2 $\triangle \mathrm{C} 3$

Figure 3.8: Batch experiment plate count data for three separate cultures (C5-C7); initial acetate concentration of $350 \mathrm{ppm}$. 
Protein concentrations of batch cultured cells were determined in order to relate the protein concentrations from core and bead columns to the total number of cells in these columns. Since the cells adhere tightly adhering to the grain surfaces in the columns, the calculation of growth could not be done by plate counts. Protein extraction in strong base (e.g. $1 \mathrm{~N} \mathrm{NaOH}$ ) and protein measurements were a surrogate method for measuring the number of cells in the columns. Separate measurements of protein concentrations in batch cultures revealed that the average protein concentration was $4.53 \times 10^{-10} \mu \mathrm{g}$ per cfu. Therefore, the total protein extracted from the columns divided by this number would equal the colony-forming units (roughly equal to the number of cells) on the columns.

Figure 3.9 and 3.10 plot the acetate concentrations vs. time in batch cultures of $P$. aeruginosa. In four experiments (Figure 3.9) the initial concentration of $0.1 \%$ sodium acetate in the growth medium corresponds to $\sim 700 \mathrm{ppm}$ acetate. The majority of growth is occurring between 10 and 40 hours (see Fig. 3.7 and 3.8 above), and this corresponds to the consumption of acetate. At stationary phase the concentration of acetate levels out at $\sim 100 \mathrm{ppm}$ in batch experiments $\mathrm{C} 1-\mathrm{C} 4$. However acetate concentration stabilizes near zero in batch experiments $\mathrm{C} 5-\mathrm{C} 7$ which had an initial acetate concentration of $350 \mathrm{ppm}$ (Figure 3.10). It was unclear which concentration (0 or $100 \mathrm{ppm}$ ) would be expected in the column effluent during the flow experiments (chapter 5). The rate of acetate consumption goes through a maximum during the exponential growth phase. This behavior has implications for what to expect in corefloods (Chapter 5). The effluent acetate concentration would exhibit a minimum, then increase to a plateau where the cells reach the stationary phase of growth. 


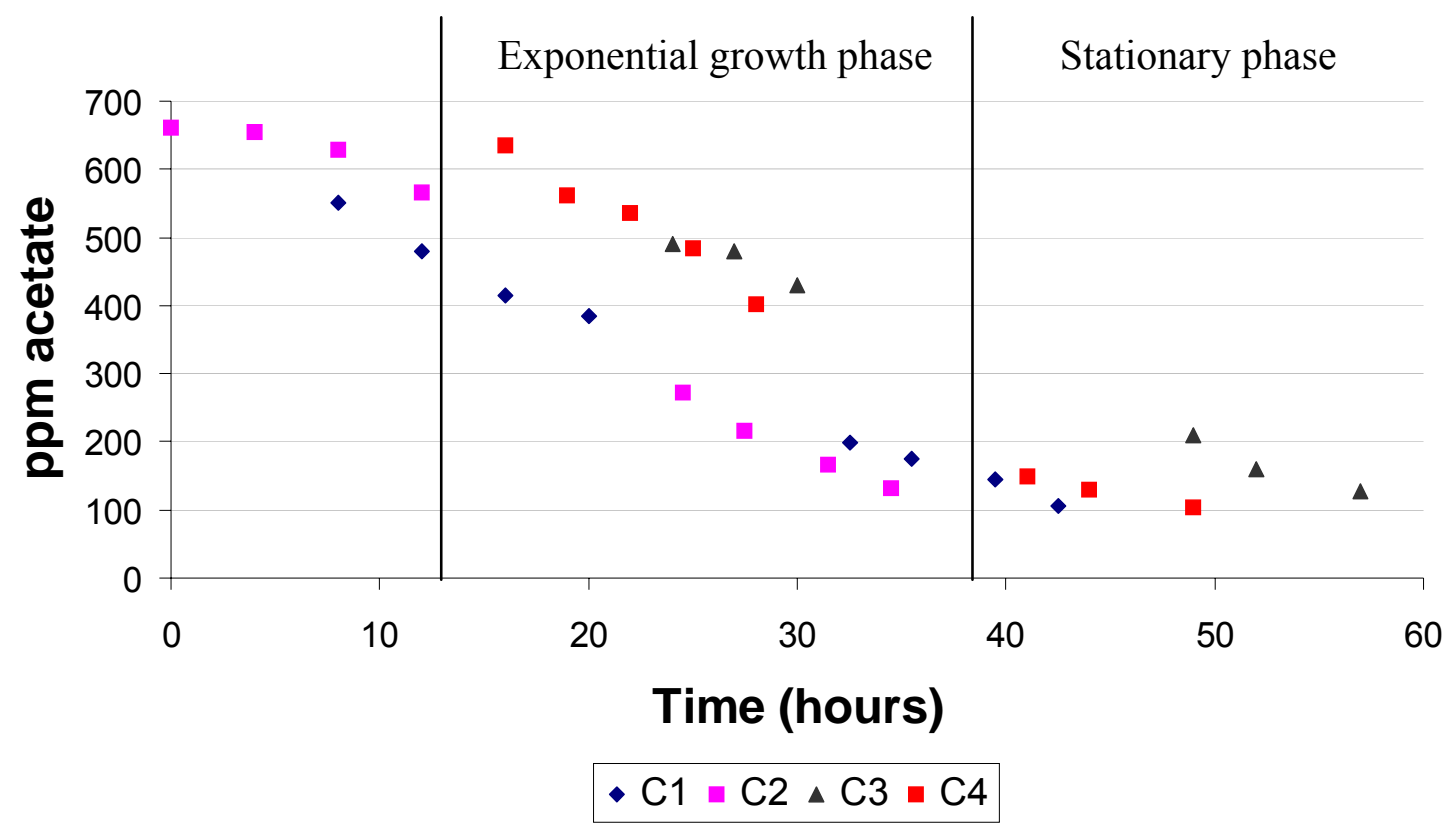

Figure 3.9: Batch experiment acetate utilization for four separate cultures (C1-C4)

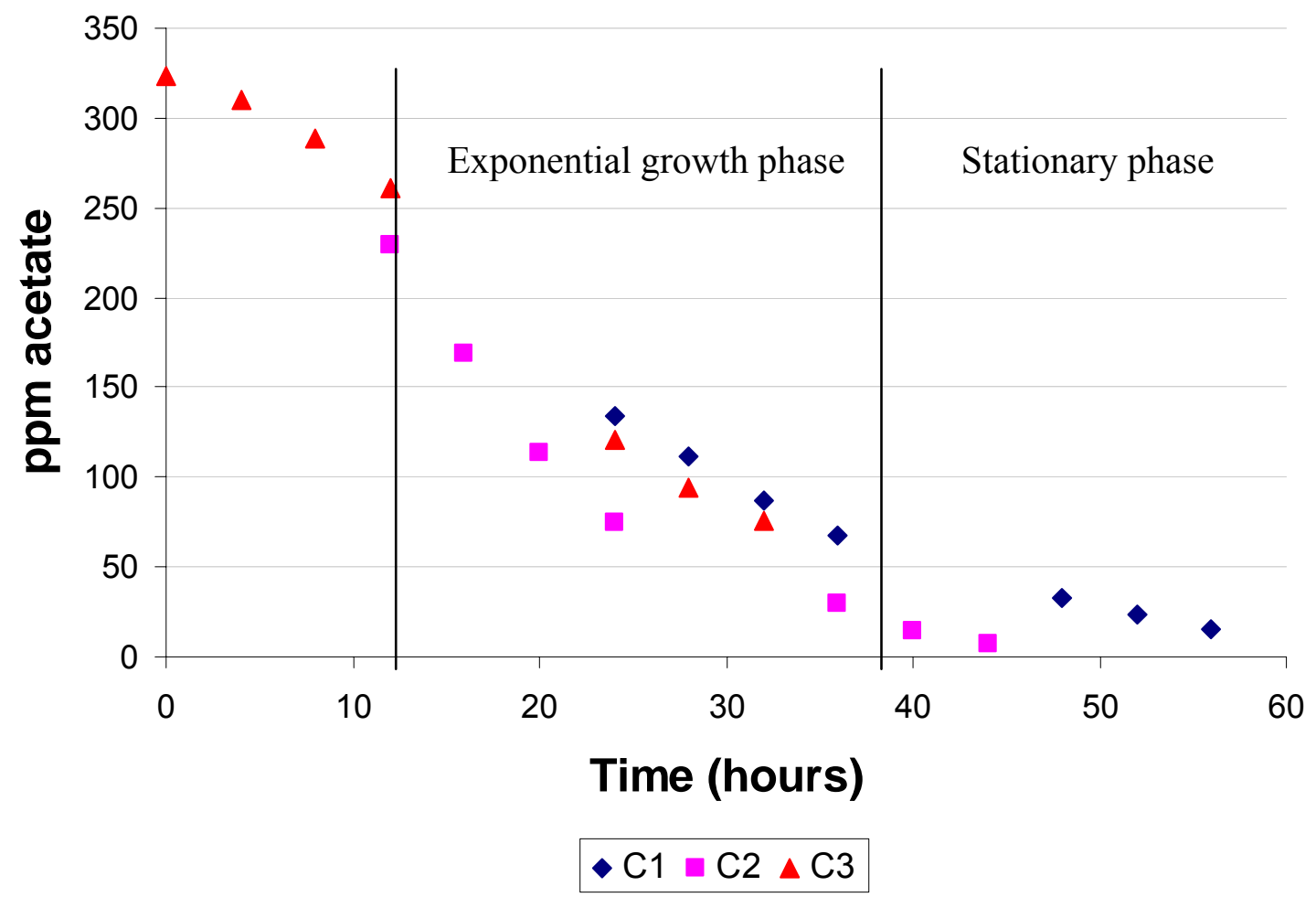

Figure 3.10: Batch experiment acetate utilization for three separate cultures (C5-C7) 


\section{Topic 1: COMPLETE CATALOG OF EXPERIMENTS -- Bead Pack Results}

Permeability reduction is defined by $\mathrm{K} / \mathrm{K}_{0}$, where $\mathrm{K}$ = permeability and $\mathrm{K}_{\mathrm{o}}=$ permeability of the bead pack pre biofilm. The permeability is determined from measurements of pressure drop across the core with aerated Dworkin Foster mineral medium flowing through it. Because fluid viscosity is the same for all measurements, changes in pressure drop are only the result of biofilm growth (aerobic). The mean permeability reduction of all bead pack experiments was 54\% in an average time of $6 \mathrm{PV}$ (Table 5.1). Bead packs 1-5, and 7 were injected with 700 ppm acetate at the standard flow rate of $1.28 \mathrm{~mL} / \mathrm{hr}$. Bead pack 6 was also injected with $700 \mathrm{ppm}$ acetate, but at an accelerated flow rate of $3.62 \mathrm{~mL} / \mathrm{hr}$. Tracers were injected into bead pack 7 before and after biofilm growth to reveal changes in effective porosity and dispersivity. Bead pack 8 tested the effect of a smaller injected acetate concentration, and bead pack 9 tested the ability of biofilm to divert injected fluids into a low permeability zone.

Table 5.1: Permeability reduction and petrophysical data

\begin{tabular}{|c|c|c|c|c|c|c|c|c|c|c|}
\hline Porous medium & $\begin{array}{c}\text { Flow rate } \\
\mathrm{mL} / \mathrm{hr}\end{array}$ & $\mathrm{K}_{\mathrm{O}} \mathrm{md}$ & $\mathrm{K}$ md & $\mathrm{K} / \mathrm{K}_{\mathrm{O}}$ & $\begin{array}{c}\text { Time to achieve } \\
\text { minimum } \\
\text { permeability }\end{array}$ & $\begin{array}{c}\text { Injected acetate } \\
\text { concentration } \\
\text { ppm }\end{array}$ & $\begin{array}{c}\text { Minimum effluent } \\
\text { acetate } \\
\text { concentration } \\
\text { ppm }\end{array}$ & $\begin{array}{c}\text { End of } \\
\text { experiment } \\
\text { effluent acetate } \\
\text { concentration } \\
\text { ppm }\end{array}$ & \begin{tabular}{|c|} 
End of \\
experiment \\
effluent acetate \\
concentration \\
divided by \\
injected \\
concentration
\end{tabular} & $\begin{array}{l}\text { Staining } \\
\text { method }\end{array}$ \\
\hline Bead pack \#1 & 1.28 & 1483 & 830 & 0.56 & $3 \mathrm{PV}^{*}, 41 \mathrm{PV}$ & 700 & $579 @ 3.5 \mathrm{PV}$ & 649 & 0.93 & TTC \\
\hline Bead pack \#2 & 1.28 & 1414 & 650 & 0.46 & $12.5 \mathrm{PV}$ & 700 & $428 @ 37$ PV & 428 & 0.61 & NBT \\
\hline Bead pack \#3 & 1.28 & 1375 & 1100 & 0.80 & $3.5 \mathrm{PV}$ & 700 & $584 @ 13$ PV & 642 & 0.92 & NBT \\
\hline Bead pack \#4 & 1.28 & 1123 & 348 & 0.31 & $10 \mathrm{PV}$ & 700 & $545 @ 11 \mathrm{PV}$ & 669 & 0.96 & Bradford \\
\hline Bead pack \#5 & 1.28 & 1413 & 622 & 0.44 & $6 \mathrm{PV}^{*}, 23 \mathrm{PV}$ & 700 & $577 @ 15 \mathrm{PV}$ & 616 & 0.88 & Bradford \\
\hline Bead pack \#6 & 3.62 & 1492 & 761 & 0.51 & $4 \mathrm{PV}$ & 700 & $613 @ 14$ PV & 655 & 0.94 & Bradford \\
\hline Bead pack \#7 & 1.28 & 1446 & 651 & 0.45 & 3.5 PV & 700 & $548 @ 11 \mathrm{PV}$ & 631 & 0.90 & Bradford \\
\hline Bead pack \#8 & 1.28 & 1420 & 838 & 0.59 & $5 \mathrm{PV}$ & 300 & $220 @ 12 \mathrm{PV}$ & 264 & 0.88 & none \\
\hline Bead pack \#9 & 1.28 & 10000 & 1100 & 0.11 & $7 \mathrm{PV}$ & 700 & $372 @ 12$ PV & 525 & 0.75 & Bradford \\
\hline Core A & 1.28 & 62 & 17 & 0.28 & $6 \mathrm{PV}$ & 700 & 578 & & & Uranium \\
\hline Core B & 1.28 & 45 & 25 & 0.55 & $48 \mathrm{PV}$ & 700 & $521 @ 15$ PV & 661 & 0.94 & none \\
\hline
\end{tabular}

Note: starred values for bead packs 1 and 5 are for initial reduction. In these two bead packs reductions were experienced in two distinct stages.

\section{Bead Pack 1}

Bead pack $1\left(\mathrm{~K}_{\mathrm{o}}=1483 \mathrm{md}\right)$ had a final permeability reduction of $44 \%\left(\mathrm{~K} / \mathrm{K}_{\mathrm{o}}=0.56\right.$;

Figure 5.1). Permeability reduction occurred in two distinct stages in this experiment. In 
the first stage, permeability was reduced by $12 \%\left(\mathrm{~K} / \mathrm{K}_{\mathrm{o}}=0.88\right)$ during the first $3 \mathrm{PV}$ and remained steady from $3 \mathrm{PV}$ to $35 \mathrm{PV}$. After $35 \mathrm{PV}$, permeability decreased again, from $12 \%$ reduction to the final reduction of $44 \%$. Effluent acetate concentrations in this bead pack decreased in the early part of the experiment, then slowly increased over the period of constant permeability. After $35 \mathrm{PV}$, when permeability began to decrease again, there was no corresponding change in acetate levels. At the end of the experiment, measured effluent acetate concentration was $93 \%$ of the injection concentration. The second reduction in permeability may have occurred without a significant increase in biomass since acetate concentrations remained steady or slightly increased. It is possible that minor growth in the column's exit frit could have decreased the measured permeability without substantial acetate consumption.

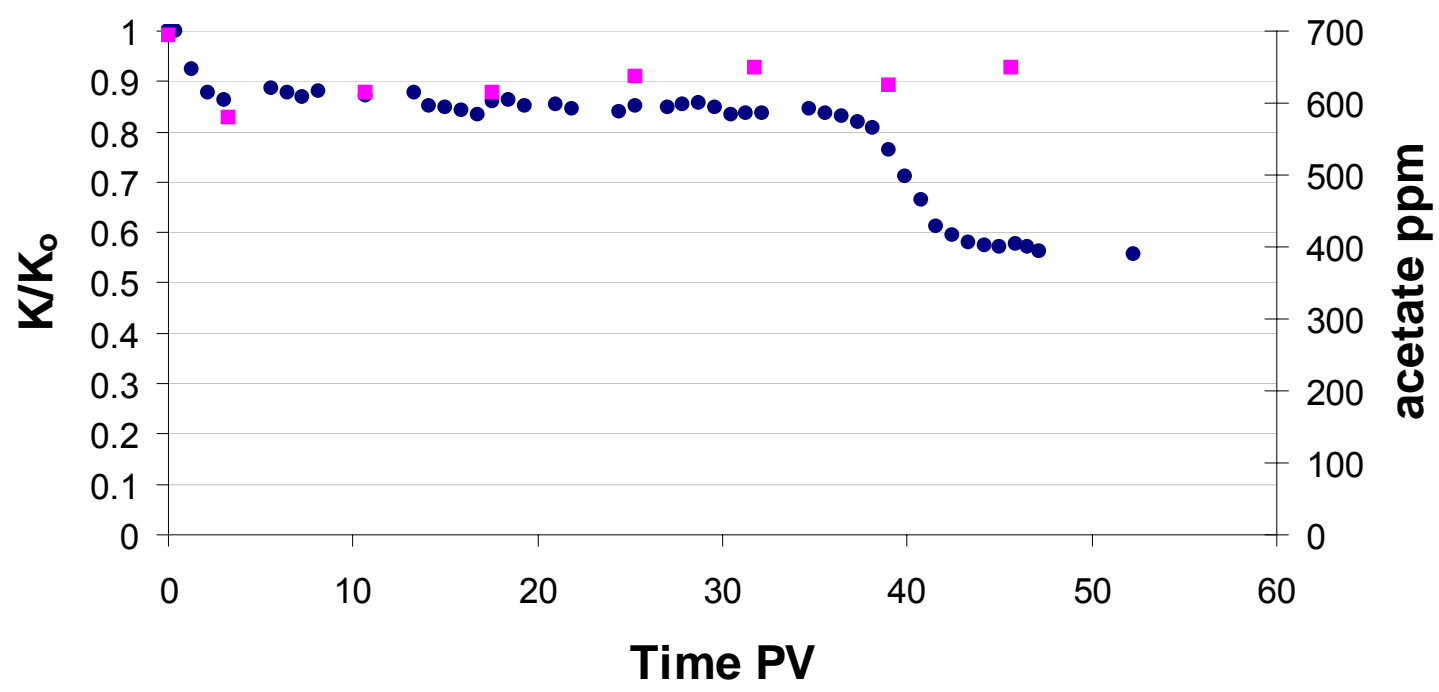

- permeability - acetate

Figure 5.1: Permeability reduction and effluent acetate concentration for bead pack 1

Bead pack 1 biomass was dyed with the TTC solution (chapter 4). After injection of the dye a region of biomass $2-3 \mathrm{~cm}$ in length was observed on the influent side of the bead 
pack, suggesting that heavier growth occurred at the front (influent side) of the column. This bead pack was injected with the acrylamide/bis-acrylamide polymer solution, allowed to gel, and then extruded from the glass column. Slices of the extruded bead pack were viewed under a microscope. The staining indicated that microbial colonization was in isolated patches (Figure 5.2). It was probable that the sparsity of biofilm was due to the inadvertent stripping of biofilm from the column during the high rate injection of the viscous polymer solution.

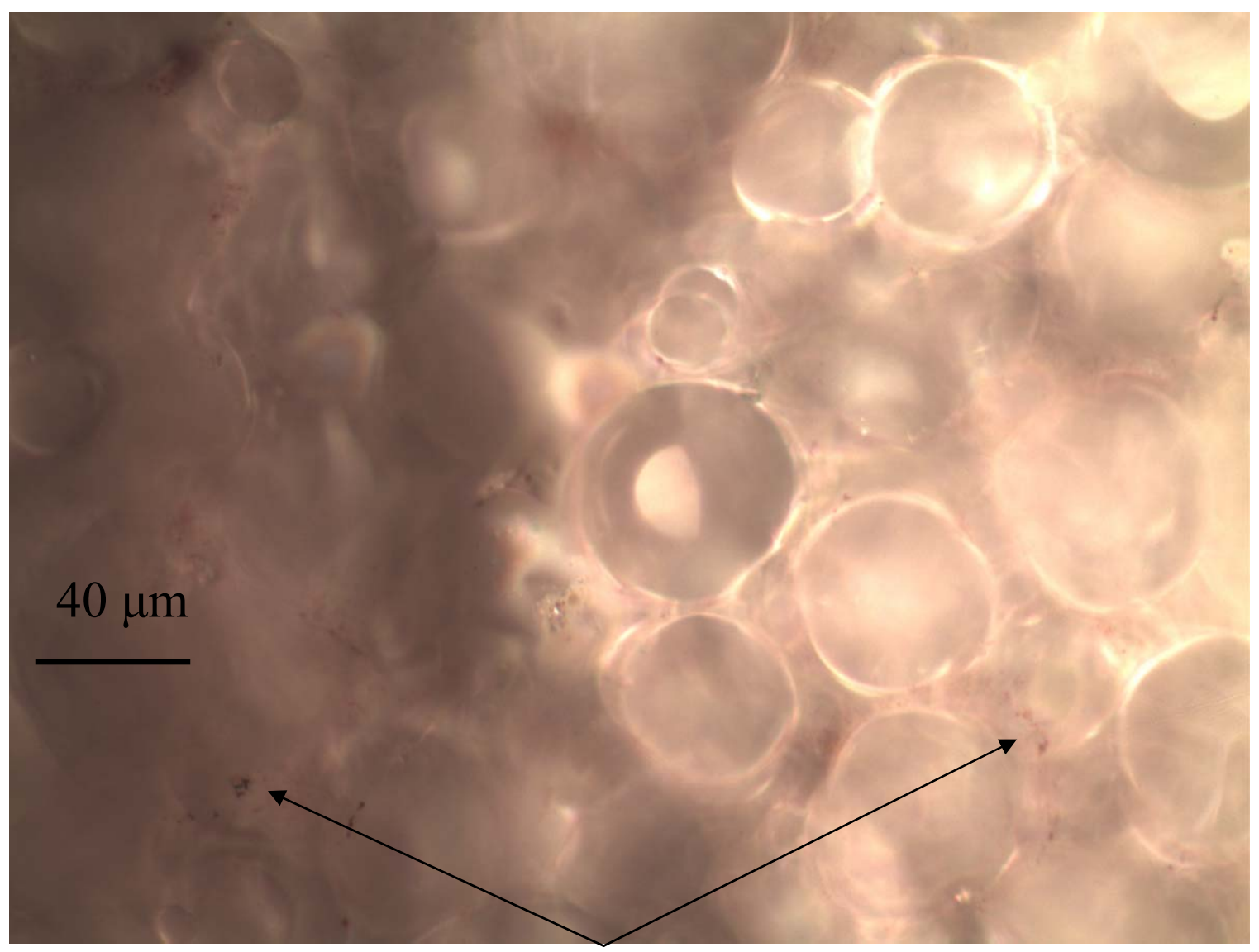

Figure 5.2: Bead pack 1 dyed with TTC solution at 500X magnification. Small microbial colonies appear as red dots. The overall reddish tint is an artifact of the visualization process and not due to the red formazan product of TTC reduction. 


\section{Bead Pack 2}

Bead pack $2\left(\mathrm{~K}_{\mathrm{o}}=1414 \mathrm{md}\right)$ had a final permeability reduction of $54 \%\left(\mathrm{~K} / \mathrm{K}_{\mathrm{o}}=0.46\right.$; Figure 5.3). This final reduction was reached in a time of $13 \mathrm{PV}$. The permeability reduction fluctuated between $54 \%$ and $48 \%$ until the termination of the experiment at 45 PV. Presumably microbial growth has ceased when the permeability stops changing. However, acetate consumption during this experiment continued nearly the entire time, even after permeability becomes constant after approximately $17 \mathrm{PV}$. The lowest measured effluent acetate concentrations (61\% of injection concentration) are at the end of the experiment. This core is anomalous in that measured effluent acetate concentration does not increase after the biofilm ceases to grow. It was not apparent what caused this anomalous behavior in this experiment. This could be attributable to microbial contamination of the influent reservoir. If this were the case, the injected acetate concentration would be less than $700 \mathrm{ppm}$ due to the consumption by the microbes in the influent reservoir.

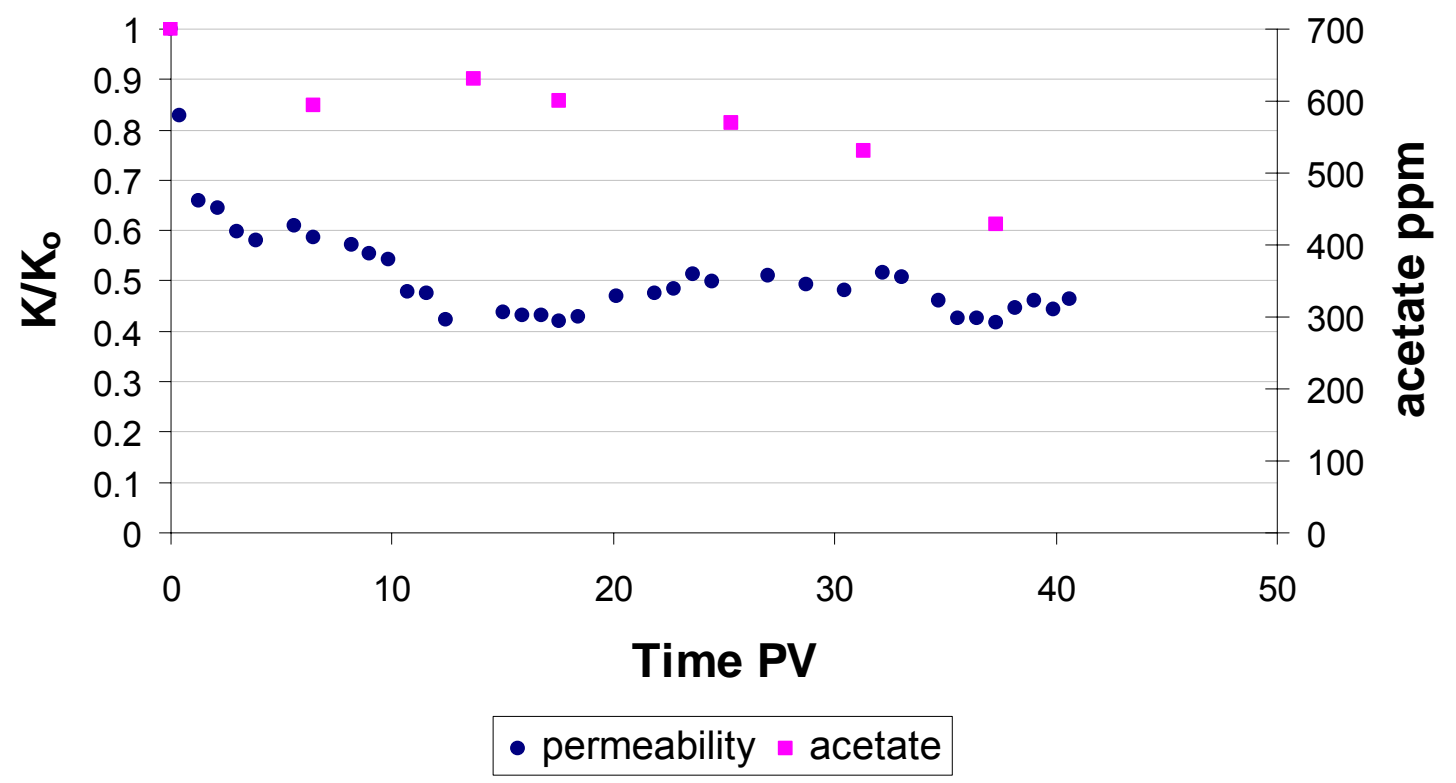

Figure 5.3: Permeability reduction and effluent acetate concentration for bead pack 2 
Bead pack 2 was injected with a NBT dye (chapter 4) to stain the microbes, but for some reason the NBT was not reduced to insoluble, colored formazan. Therefore, no imaging of biofilm was performed on this column. It is possible that NBT was competing with molecular oxygen for reducing equivalents in the respiratory chain and ancillary dehyrogenases. As long as oxygen is present, the preferred process is reduction of oxygen to water and not the reduction of NBT. Subsequent bead pack experiments using NBT were modified with the sparging of the injected NBT solution with nitrogen in order to exclude oxygen.

\section{Bead Pack 3}

Bead pack $3\left(\mathrm{~K}_{\mathrm{o}}=1375 \mathrm{md}\right)$ had a permeability reduction of $20 \%\left(\mathrm{~K} / \mathrm{K}_{\mathrm{o}}=0.80\right.$; Figure 5.4). This reduction was much less than the other experiments. The reduction was complete in a time of $3.5 \mathrm{PV}$, with no significant change in permeability over the next 20 $\mathrm{PV}$, therefore the experiment ended after $25 \mathrm{PV}$ injected. Acetate concentration decreased until $13 \mathrm{PV}$, then gradually increased so that effluent acetate concentration was $92 \%$ of the injection acetate concentration at the end of the experiment.

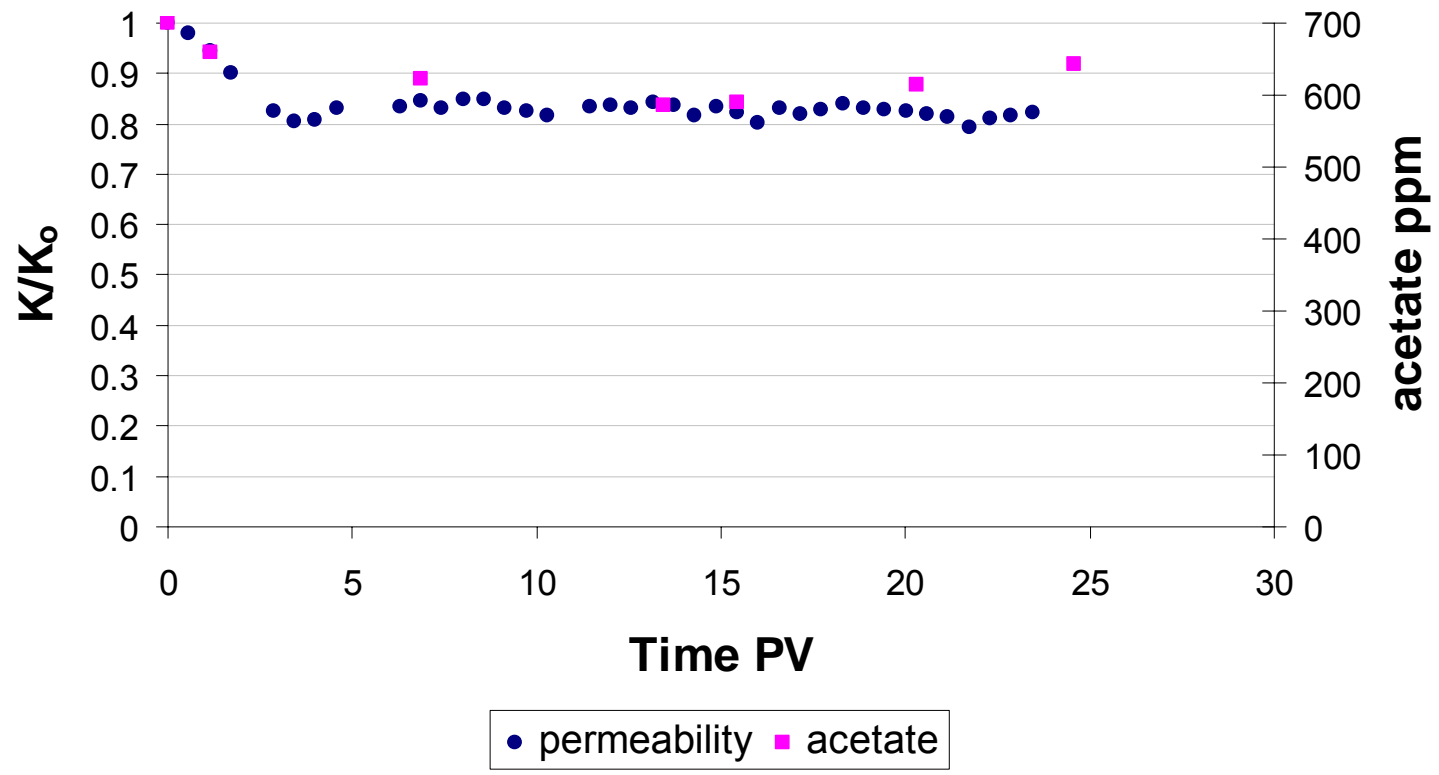

Figure 5.4: Permeability reduction and effluent acetate concentration for bead pack 3 
Bead pack 3 was stained blue/purple with the NBT solution. Stained growth was observed on both the influent and effluent ends, but not in the middle section of the bead pack. Low magnification microscopy (100X) of the influent end slices showed growth throughout the thin section (Figure 5.5). Imaging of individual beads at higher magnification (500X) shows that beads were coated with microbial cells (Figure 5.6). The general scattered distribution of growth throughout the column is consistent with the small (20\%) permeability reduction.

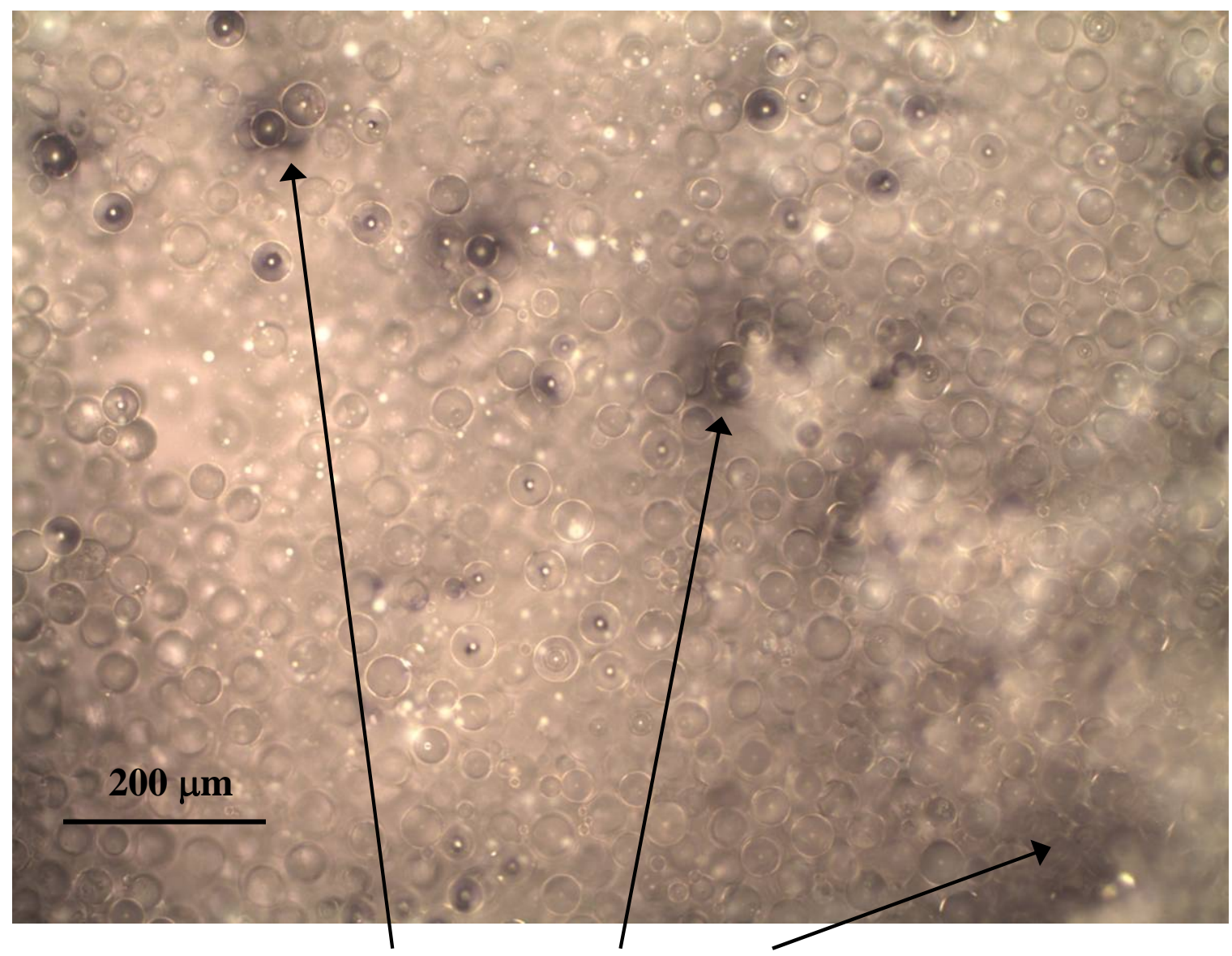

Figure 5.5: Bead pack \#3 stained with NBT solution magnified 100X. Note sparse pockets of black stained cells. 


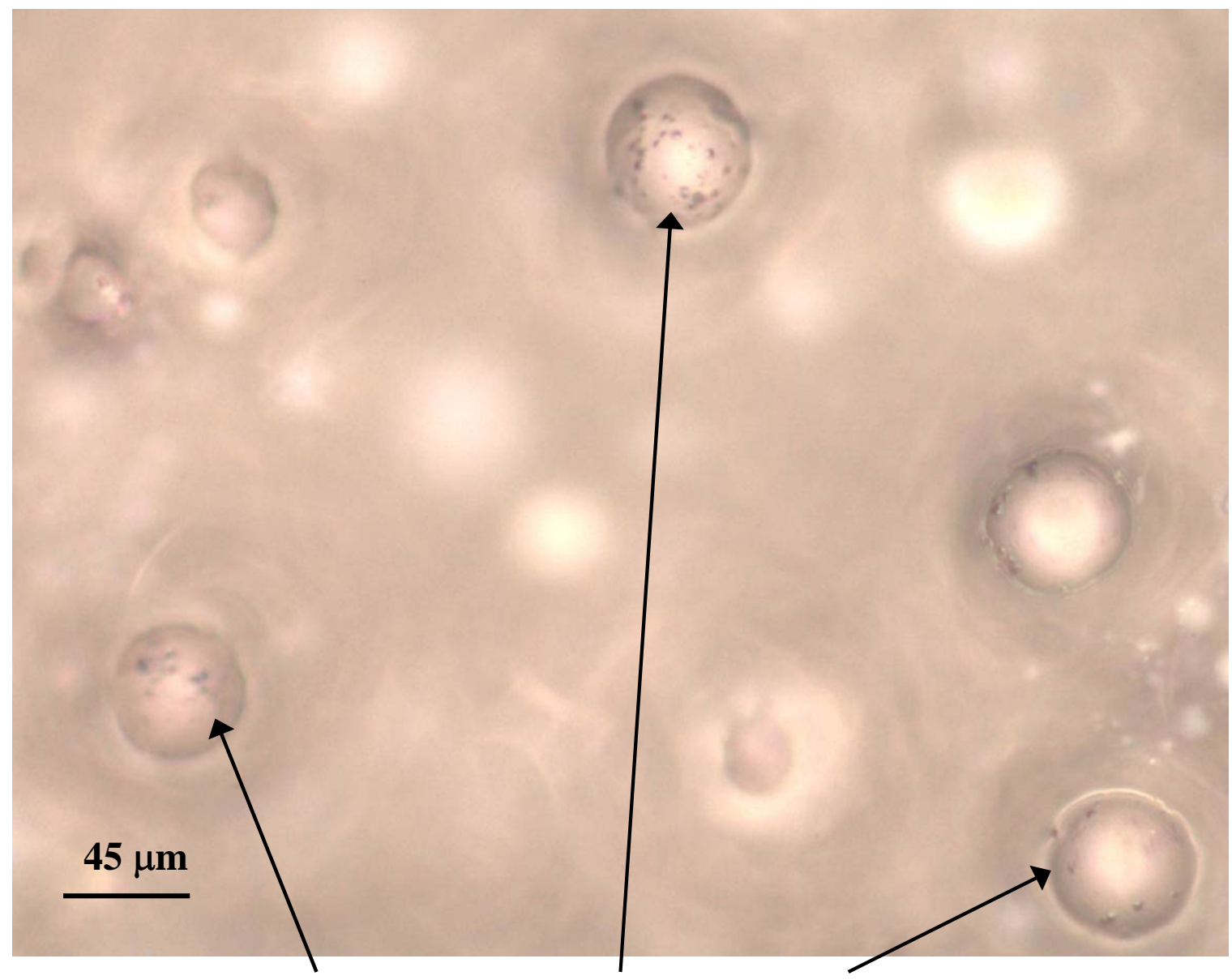

Figure 5.6: “Granular” coating of microorganisms stained with NBT in Bead pack \#3. 500X magnification.

\section{Bead Pack 4}

Bead pack $4\left(\mathrm{~K}_{\mathrm{o}}=1483 \mathrm{md}\right)$ had a large permeability reduction of $69 \%\left(\mathrm{~K} / \mathrm{K}_{\mathrm{o}}=0.31\right.$; Figure 5.7). Permeability reduction occurred rapidly from the start of the experiment until $8 \mathrm{PV}$. After $8 \mathrm{PV}$, permeability remained constant through the end of the experiment ( $\sim 30$ $\mathrm{PV})$. Acetate concentrations decreased at the beginning of the experiment until a time of $11 \mathrm{PV}$ when measured effluent acetate was at the lowest measured concentration. After $11 \mathrm{PV}$ acetate concentrations gradually increased back to $96 \%$ of the injection concentration at the end of the experiment. 


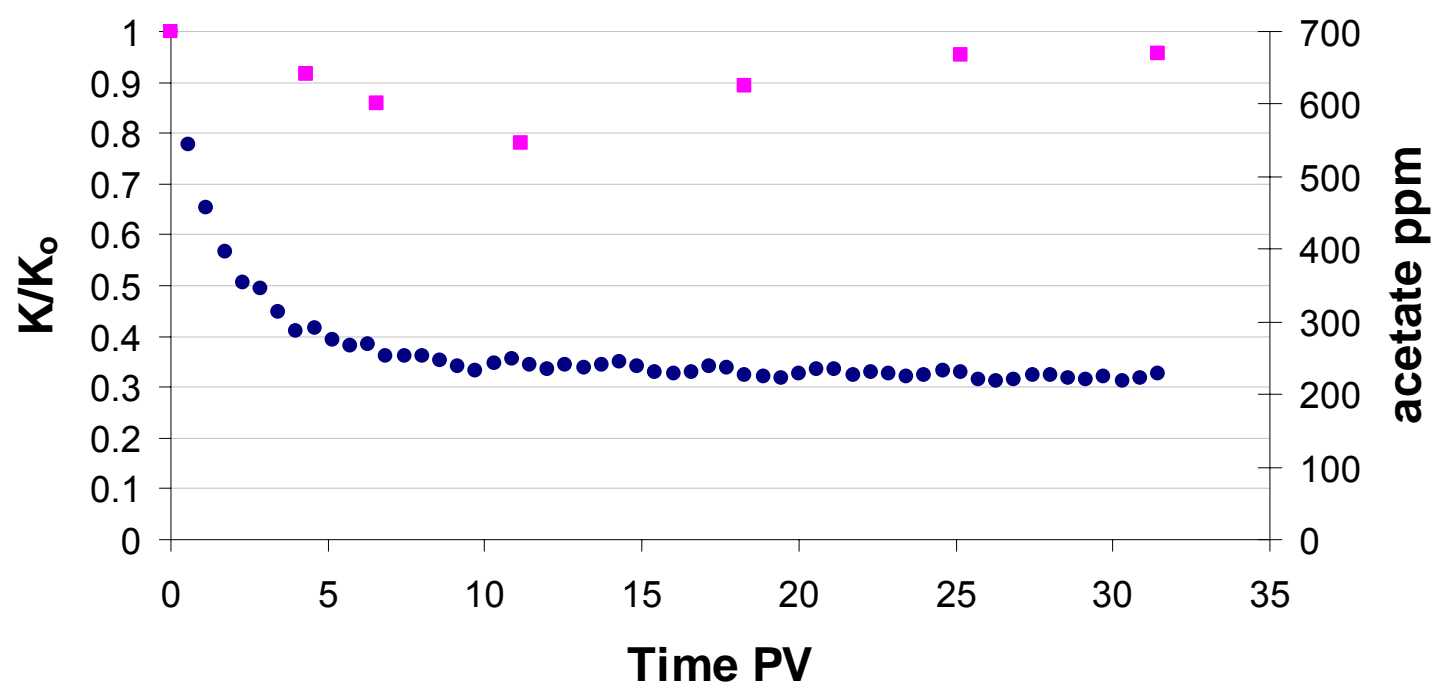

- permeability - acetate

Figure 5.7: Permeability reduction and effluent acetate concentration for bead pack 4

Bead pack 4 was stained with Bradford Protein Reagent which produces a more vivid, bright-blue color that is easier to see than NBT. Growth was extensive and especially heavy at both ends of the column. However, when the acrylamide/bis-acrylamide monomer solution was injected rapidly into the column in preparation of sectioning and microscopic examination, much of the blue-stained biomass was eluted except for some at the influent end. Therefore, microscopy on this column was not done. In retrospect more attention must be given to injection rates and solution viscosities in order to avoid dislodging the biofilm. Polymer injection into the post biofilm bead packs was discontinued upon discovery of the biofilm elution from the bead packs.

\section{Bead pack 5}

Bead pack $5\left(\mathrm{~K}_{\mathrm{o}}=1412 \mathrm{md}\right)$ had its permeability reduced $56 \%\left(\mathrm{~K} / \mathrm{K}_{\mathrm{o}}=0.44\right.$ Figure 5.8$)$. This permeability reduction occurred in two stages. The first and most significant reduction (43\%) took place between the start of the experiment and around $7 \mathrm{PV}$. Over the next $12 \mathrm{PV}$, permeability did not change significantly, and at $19 \mathrm{PV}$ permeability began to decrease again, ultimately reaching stabilization at $56 \%$. Effluent acetate 
concentrations in this experiment decreased from the beginning of the experiment until $16 \mathrm{PV}$ at which time they began to increase and reached a level of $88 \%$ of the injection concentration. This acetate utilization profile is similar to those of the previous experiments.

At the end of the permeability reduction (33 PV) in this experiment, flow rate was increased from $1.28 \mathrm{~mL} / \mathrm{hr}$ to $3.62 \mathrm{~mL} / \mathrm{hr}$ to test the integrity of the biofilm. Permeability reduction was measured for the new flow rate and plotted as permeability2 in Figure. 5.8. No change in measured permeability occurred as several PV were pumped through the bead pack at the increased flow rate. Biofilm was maintained and was not affected by the higher shear forces of the increased flow rates. This suggests that the existing biomass is not overly sensitive to shear stress in this small range of flow rates, however injection of the viscous polymer solution at very high flow rates $(600 \mathrm{~mL} / \mathrm{hr})$ was successful in stripping the biofilm from bead packs 1-4. This is documented further in Chapter 7.

When flow rate was increased and no permeability change occurred, it indicates that once growth reaches a steady state, additional biomass is not formed by the increase in nutrient flux through the column. This implies that the injected solution is not limiting with respect to nutrients and other growth essentials (e.g., $\mathrm{O}_{2}$ in aerobic growth).

Effluent acetate concentrations did not in any experiment reach the injected levels after the biofilm had stopped growing (inferred by no further permeability reduction). Most of the experiments reached an effluent acetate concentration of approximately $90-95 \%$ of the injection concentration (Table 5.1). Once the microbes reached a population density in the pore space at which they could no longer multiply, the microbial activity switched from a metabolic to a catabolic state. In a catabolic state, acetate is consumed by the cells for energy and maintenance in contrast to a metabolic state where acetate gives the cells the energy to grow and divide. 


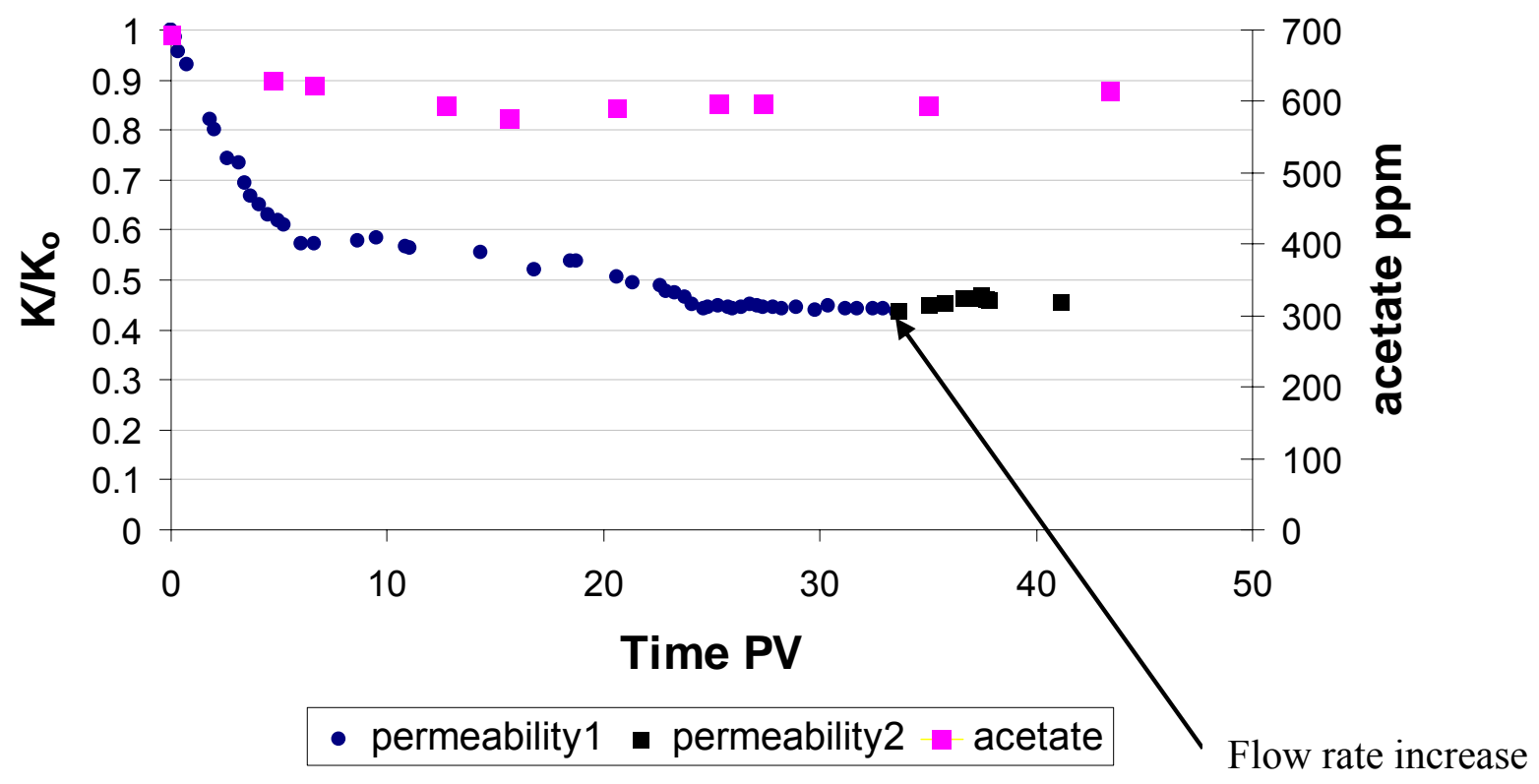

Figure 5.8: Permeability reduction and effluent acetate concentrations for bead pack 5

An image of the bead pack after staining with Bradford Protein Reagent before the beads were extracted shows intense growth throughout but especially at the ends (Figure 5.9). The micrograph of beads in Figure 5.10 shows growth throughout a cross section in the middle of the bead pack. The micrograph of beads in Figure 5.11 shows extensive biofilm on the beads and illustrates that pore-filling growth explains the observed overall $69 \%$ permeability reduction. Microbial protein (cells) stain a blue color with the Bradford Protein Reagent. The extra-cellular microbial byproducts are capsular in nature and are composed of polymers and polysaccharides, which are generated by the microbes in-situ. The extra-cellular materials are continuous in nature and encompass the cells as well as the beads. The combination of cells and extra-cellular material fill pore throats and are effective in reducing permeability as evidence in this bead pack. This will be discussed at greater length in Chapter 7. 


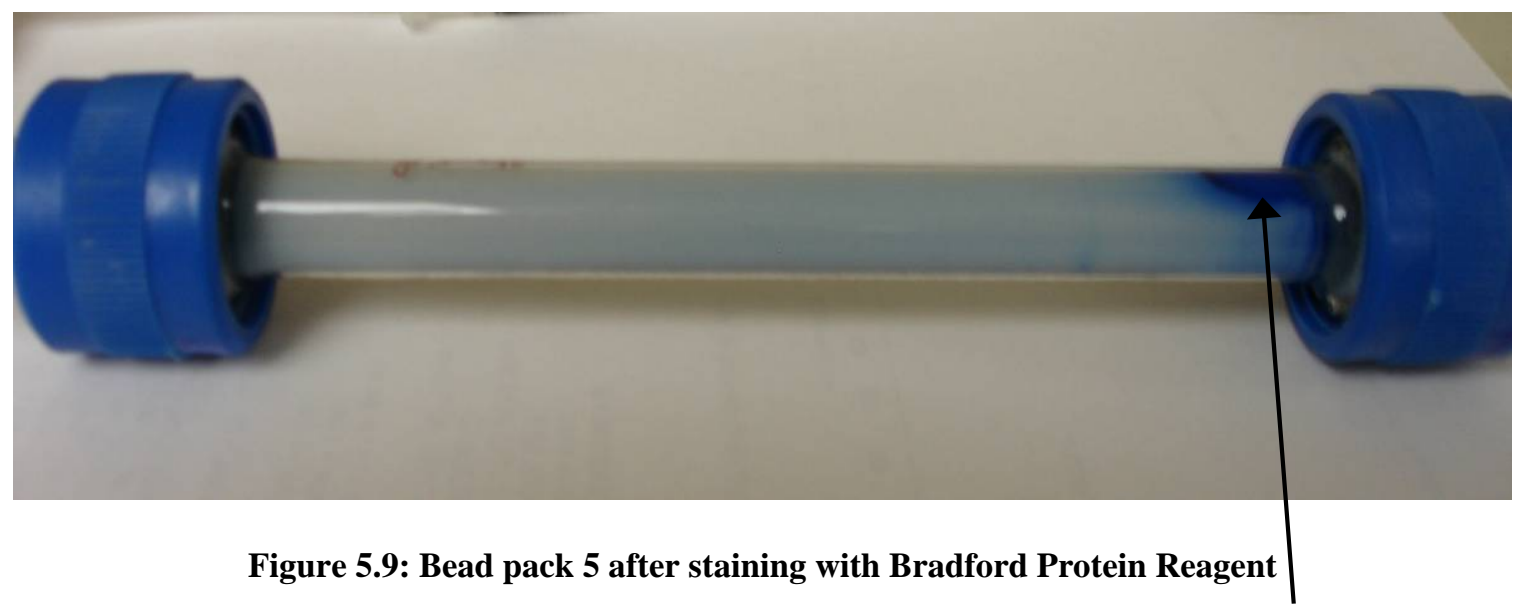

Notice the blue hue of the column and the heavy growth at the influent end.

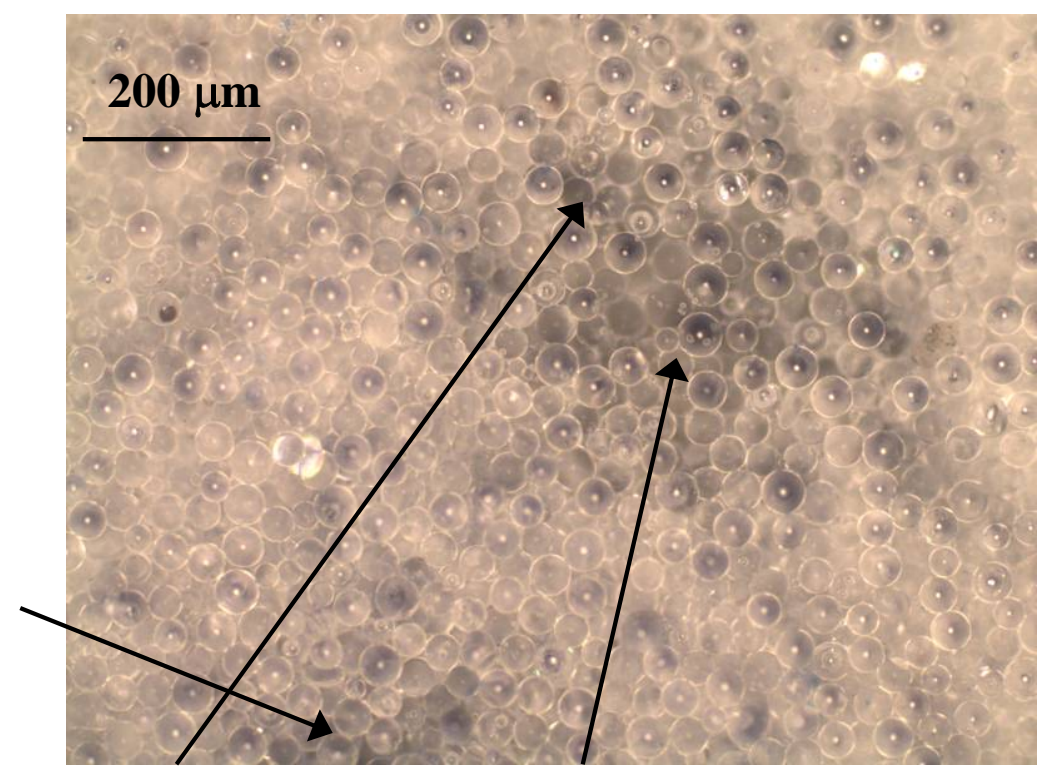

Figure 5.10: Microbial colonization shows clusters of growth. 100X magnification 


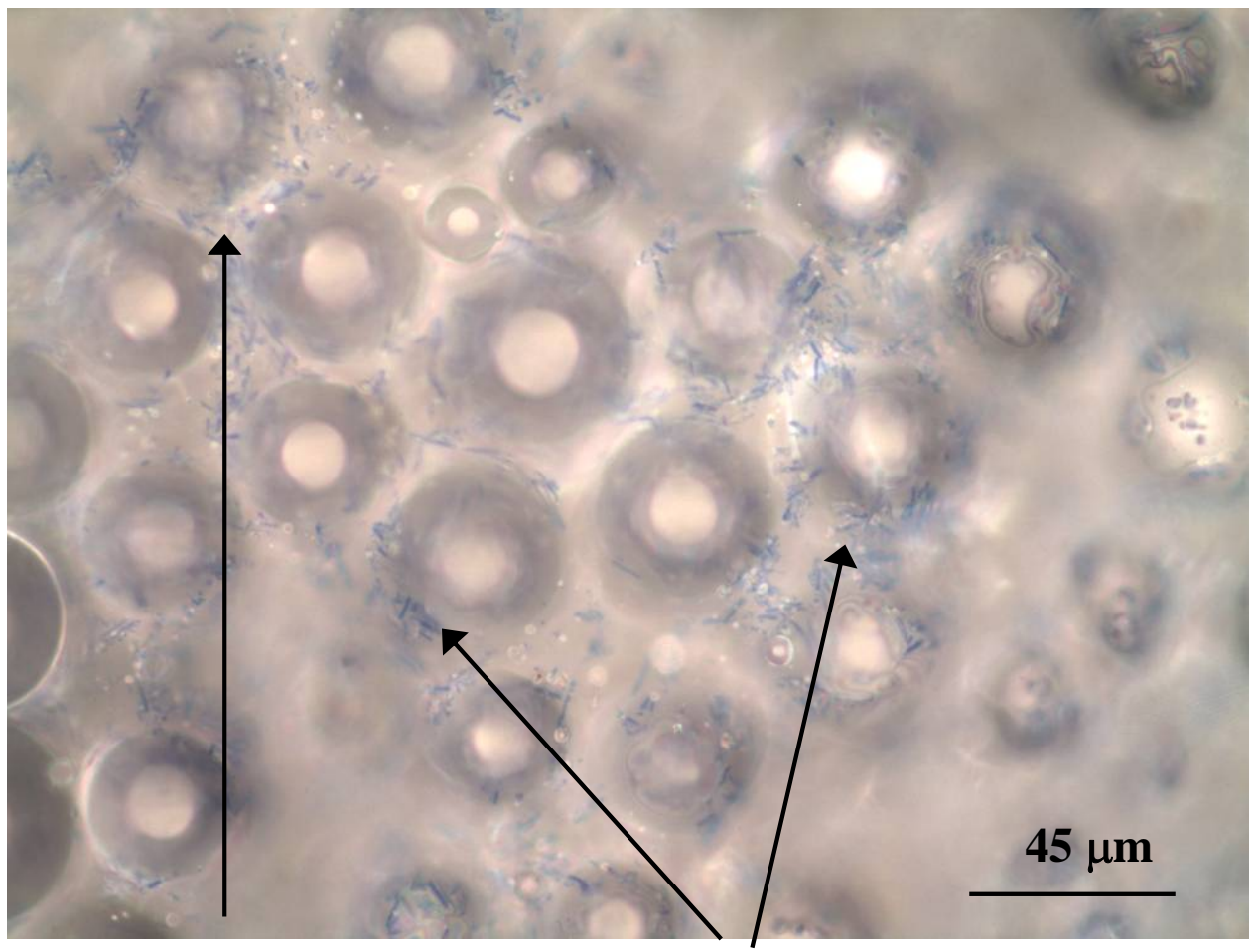

Figure 5.11: Pore-filling growth observed in bead pack 5. Cells are blue, extra-cellular products encapsulate grains and cells. 500X magnification

\section{Bead Pack 6}

Bead pack 6 was run at an accelerated flow rate of $3.62 \mathrm{~mL} / \mathrm{hr}$ compared with bead packs $1-5$ which were pumped at $1.28 \mathrm{~mL} / \mathrm{hr}$. A different permeability symbol (closed squares) is used in Figure 5.12 to distinguish the faster rate from previous bead pack results. Permeability reduction occurred very rapidly and reached $49 \%\left(\mathrm{~K} / \mathrm{K}_{\mathrm{o}}=0.51\right.$; Figure 5.12) at $5 \mathrm{PV}$. Like most previous experiments, the permeability reduction reached a stabilization level and remained constant until the termination of the experiment. Effluent acetate concentrations dropped in the beginning of the experiment commensurate with permeability reduction and reached a minimum at $14 \mathrm{PV}$. After $14 \mathrm{PV}$, effluent acetate concentration began to increase and reached $94 \%$ of the injection concentration at $35 \mathrm{PV}$. The overall character of the acetate concentration profile is not different than that of the other bead packs. The peak utilization (corresponding to the lowest measured 
concentration) occurred at $14 \mathrm{PV}$ which was similar to the other experiments where the peak utilization was measured between 11-15 PV.

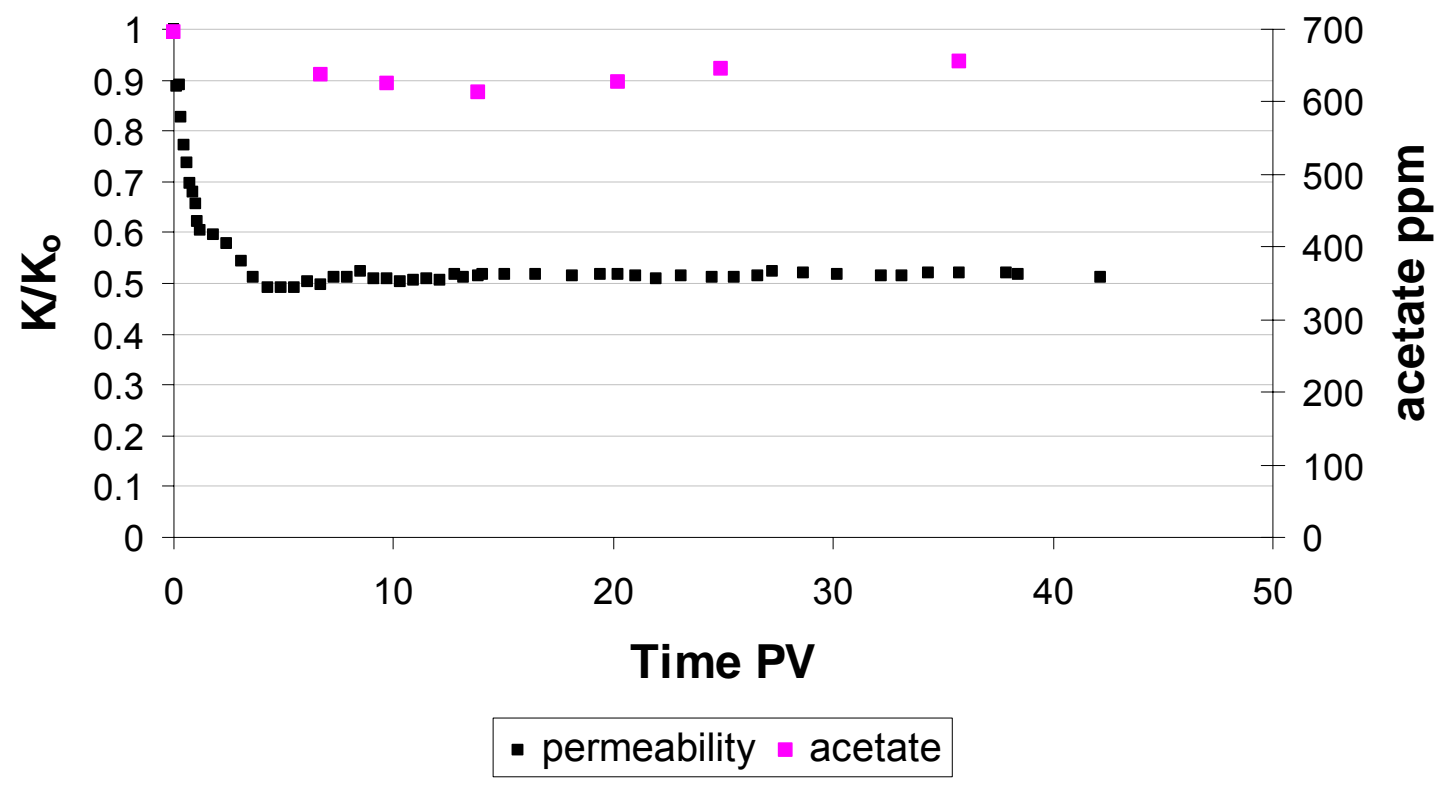

Figure 5.12: Permeability reduction and effluent acetate concentration for bead pack 6 (accelerated flow rate)

\section{Bead Pack 7}

Bead pack 7 was run at the original $1.28 \mathrm{~mL} / \mathrm{hr}$ flow rate with $700 \mathrm{ppm}$ acetate. Tracer tests were conducted to assess differences in effective porosity and dispersivity before and after biofilm growth. The permeability reduction was $56 \%\left(\mathrm{~K} / \mathrm{K}_{\mathrm{o}}=0.44\right.$; Figure 5.13) which occurred during the first 3.5 PV. No significant changes in permeability happened between 3.5 PV and 30 PV, the end of the experiment. Effluent acetate concentrations reached a minimum at $11 \mathrm{PV}$ whereupon there was a gradual increase. These results are similar to most of the other experiments. That is, acetate levels increased following the end of the permeability reduction, and at the end of the experiment the effluent acetate concentration was $90 \%$ of the injection concentration. 


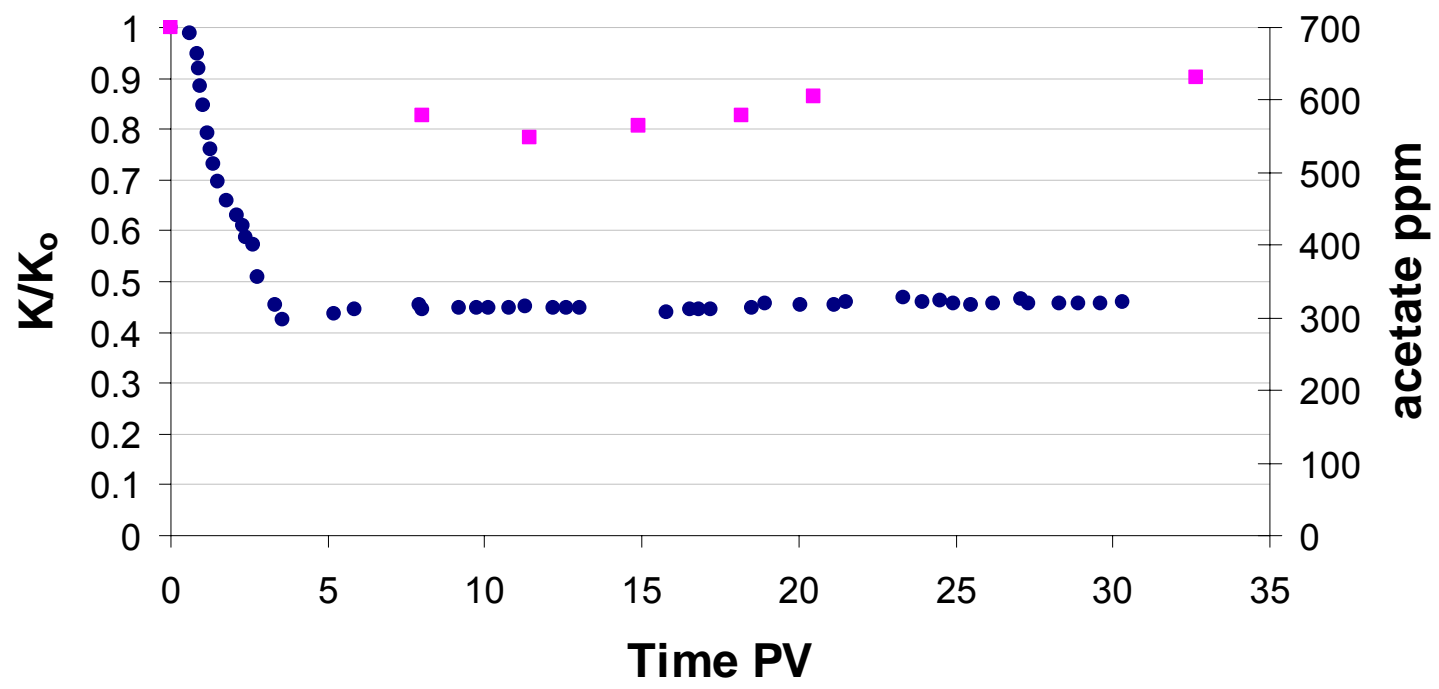

- permeability - acetate

Figure 5.13: Permeability reduction and effluent acetate concentration for bead pack 7

Tracer tests were run with a $6 \% \mathrm{NaCl}$ solution. The infinite-boundary-conditions solution to the classical 1D convection diffusion equation was fit to the measured effluent data (Figure 5.14). The change in effective porosity calculated from the convection diffusion equation assumes that microbes were solid and impermeable, that is, flow through biofilm was not possible, and diffusion of tracer into the biofilm did not occur. Later investigation shows that the biofilm is not solid and impermeable but instead semipermeable. Accounting for this would require a mass transfer coefficient that cannot be independently estimated, so this study is limited to the simplifying assumption of impermeable biofilm. Properties determined from the fit are Peclet number and effective porosity; these are shown in Table 5.2.

Table 5.2: Before and after biofilm growth tracer results for bead pack 7

\begin{tabular}{|c|r|r|r|}
\cline { 2 - 4 } \multicolumn{1}{c|}{} & $\begin{array}{c}\text { Pore space } \\
\mathrm{cm}^{3}\end{array}$ & \multicolumn{1}{c|}{$\Phi_{\text {eff }}$} & \multicolumn{1}{c|}{$\mathrm{N}_{\mathrm{pe}}$} \\
\hline before biofilm growth & 4.50 & 0.38 & 120 \\
\hline after biofilm growth & 3.69 & 0.31 & 60 \\
\hline
\end{tabular}


The tracer test indicates that a $19 \%$ reduction in effective porosity occurred with microbial colonization of the bead pack. The total pore space decreased by eight tenths of a cubic centimeter. The reduction of the Peclet number from 120 to 60 indicates that biofilm has made the bead pack more dispersive. This will be explored at greater detail in chapter 7.

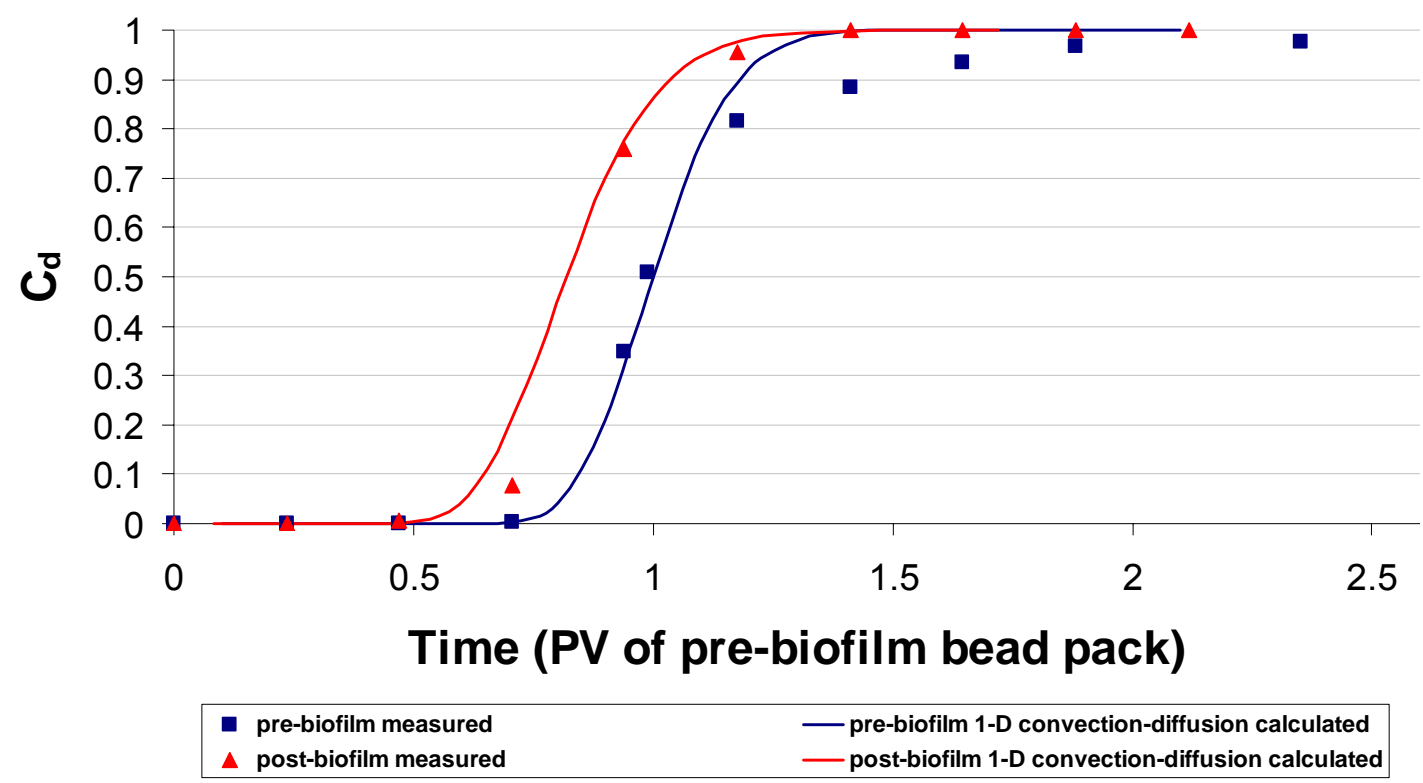

Figure 5.14: Before and after biofilm NaCl tracer test for bead pack 7. Calculated curves use 1-D convection-diffusion equation with parameters from Table 5.2.

\section{Bead Pack 8}

Bead pack 8 was run with $300 \mathrm{ppm}$ acetate concentration at the standard $1.28 \mathrm{~mL} / \mathrm{hr}$ flow rate. The purpose of this experiment was to investigate biofilm formation rate at decreased substrate concentration. Bead pack 8 had a permeability reduction of $40 \%$ $\left(\mathrm{K} / \mathrm{K}_{\mathrm{o}}=0.60\right.$; Figure 5.15) within 4.5 PV, and permeability did not change subsequently (37 PV injected). Effluent acetate concentrations decreased from the start of the experiment until $12 \mathrm{PV}$. At this point, as seen in previous experiments, effluent acetate concentrations steadily increased until the end of the experiment. Although the permeability reduction pattern was the same as in previous experiments, the percentage permeability reduction was less. However the observed permeability reduction of $40 \%$ is 
not substantially different than the results of the other experiments (bead packs 1-7) whose average permeability reduction was $54 \%$. Effluent acetate concentration at the end of the experiment was $88 \%$ of the injection concentration.

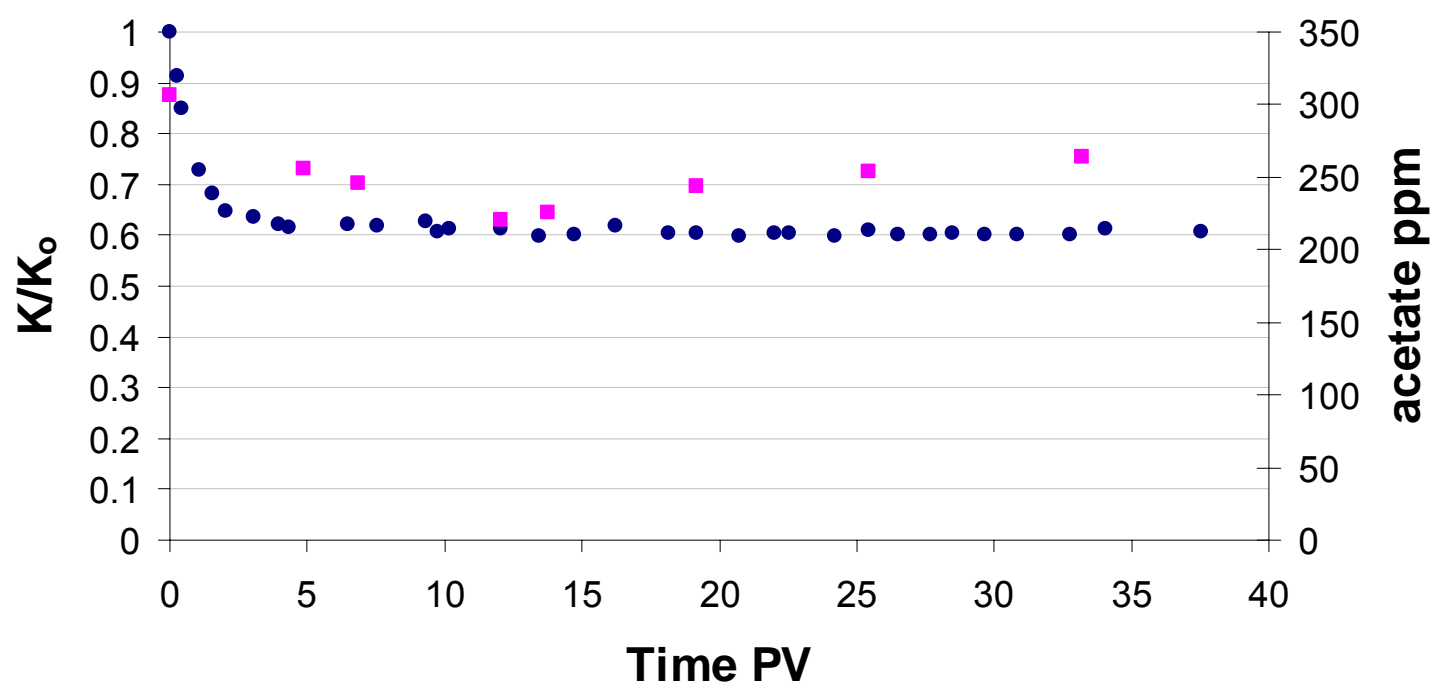

- permeability a acetate

Figure 5.15: Permeability reduction and effluent acetate concentration for bead pack 8

The amount of biomass on the column was inferred from analysis of the total protein. This was done by first removing the beads and biomass from the column and dissolving the biofilm protein in $1 \mathrm{~N} \mathrm{NaOH}$. The solution was then neutralized with a small aliquot of $12 \mathrm{~N} \mathrm{HCl}$, and protein was determined by the Bradford method. Protein determinations were also done on batch liquid cultures with known microbe concentration (colonyforming-units $/ \mathrm{mL}$ ) in order to convert the protein concentration value in the column to total number of cells expressed as colony-forming-units (cfu). The analysis revealed that $2.5 \times 10^{9}$ cfu occupied the bead pack's $4.5 \mathrm{~mL}$ interstitial fluids.

\section{Bead Pack 9}

Bead pack 9 was constructed with half of the cross-sectional area containing 38-43 $\mu \mathrm{m}$ beads and the other cross-sectional half with $180 \mu \mathrm{m}$ beads as shown in Figure 5.16. This 
was achieved by packing the column from the top with a piece of aluminum foil used to separate the two layers. The foil was removed slowly and carefully to prevent disturbing the bead size separation. Unavoidably, there was a narrow zone that was a mix of the fine and coarse beads. Flow rate was the standard $1.28 \mathrm{ml} / \mathrm{hr}$, and injected acetate concentration was $700 \mathrm{ppm}$.

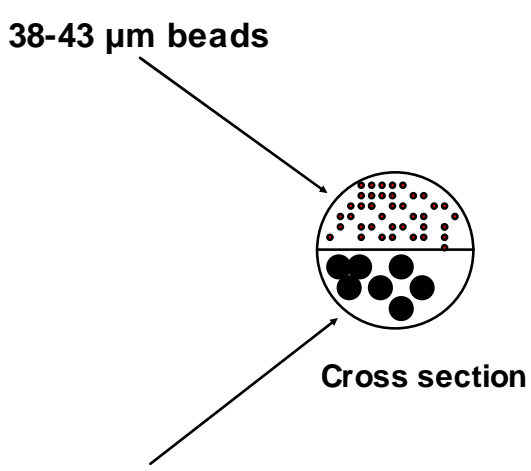

$180 \mu \mathrm{m}$ beads

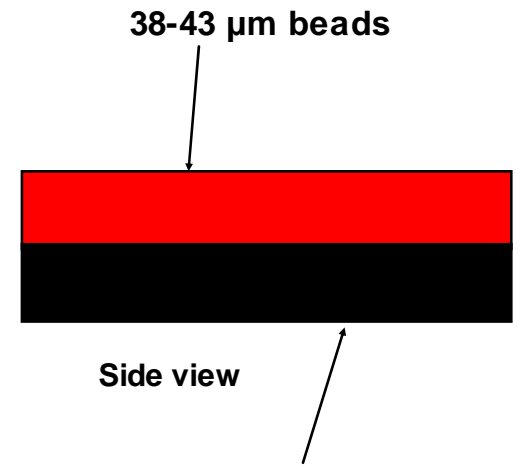

$180 \mu \mathrm{m}$ beads

Figure 5.16: Bead pack schematic 9 illustration

Bead pack 9 had a much higher initial permeability compared with bead packs 1-8. Permeability was reduced within $7 \mathrm{PV}$ by $90 \%\left(\mathrm{~K} / \mathrm{K}_{\mathrm{o}}=0.10\right.$; Figure 5.17$)$ which was greater than any of the other experiments. Effluent acetate concentrations dropped rapidly at the start of the experiment until $12 \mathrm{PV}$. Like the pattern of other experiments, acetate concentrations after $12 \mathrm{PV}$ steadily increased. Unlike previous experiments, effluent acetate stabilized at $525 \mathrm{ppm}$ which was $75 \%$ of the injection concentration. The decline in acetate was greater during the first part of the experiment. A possible explanation is the greater amount of total biomass formed in the bead pack due to the higher than average permeability reduction. 


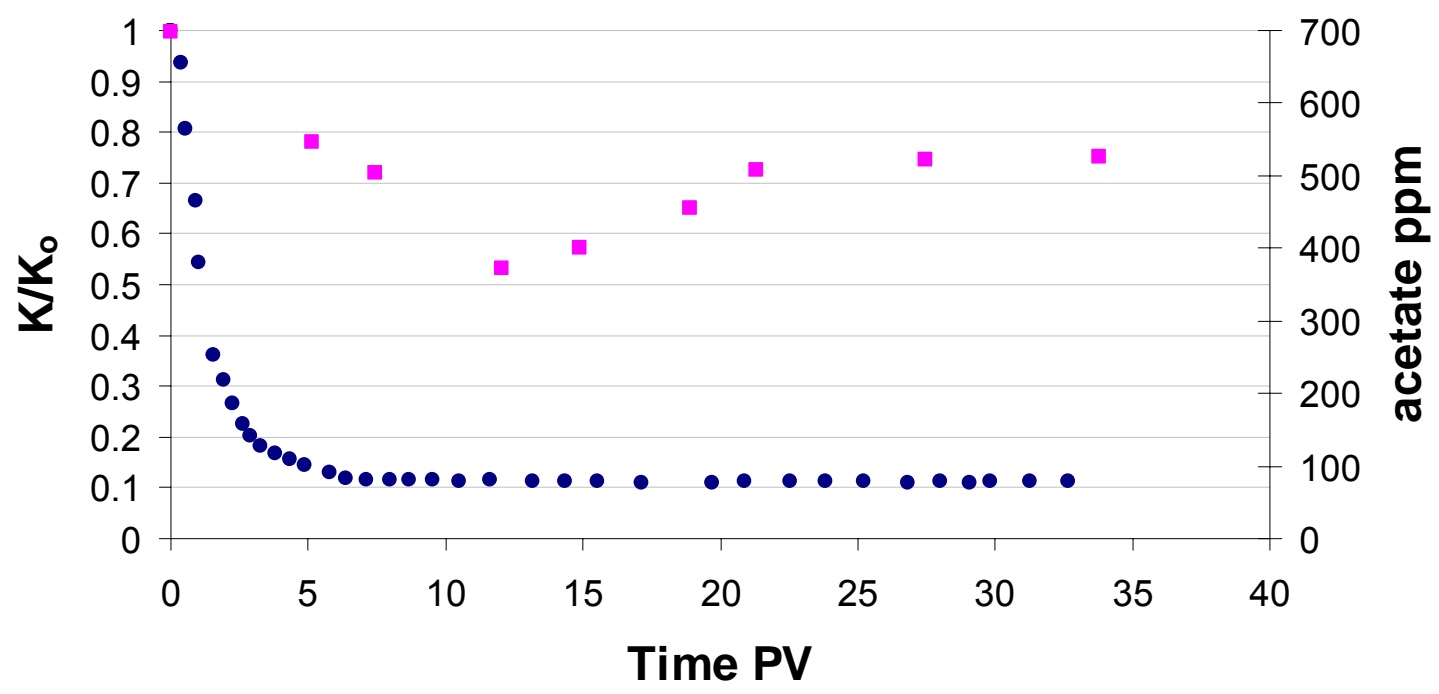

- permeability - acetate

Figure 5.17: Permeability reduction and effluent acetate concentration for bead pack 9

Microscopic examination of both bead sizes showed that biofilm was prolific in the high permeability layer (Figure 5.18a and 5.18b), but surprisingly sparse in the lower permeability layer (Figure 5.19). Before and after biofilm growth tracer tests showed significantly different behavior (Figure 5.20) which will be discussed at depth in the history matching section of Chapter 7. Time in Figure 5.20 was normalized by the pre biofilm pore volume $\left(\mathrm{PV}=\mathrm{AL} \varphi_{\text {avg }}\right)$. Breaththrough of the tracer occurred much earlier after biofilm growth due to an overall decrease in pore volume by the biofilm. 

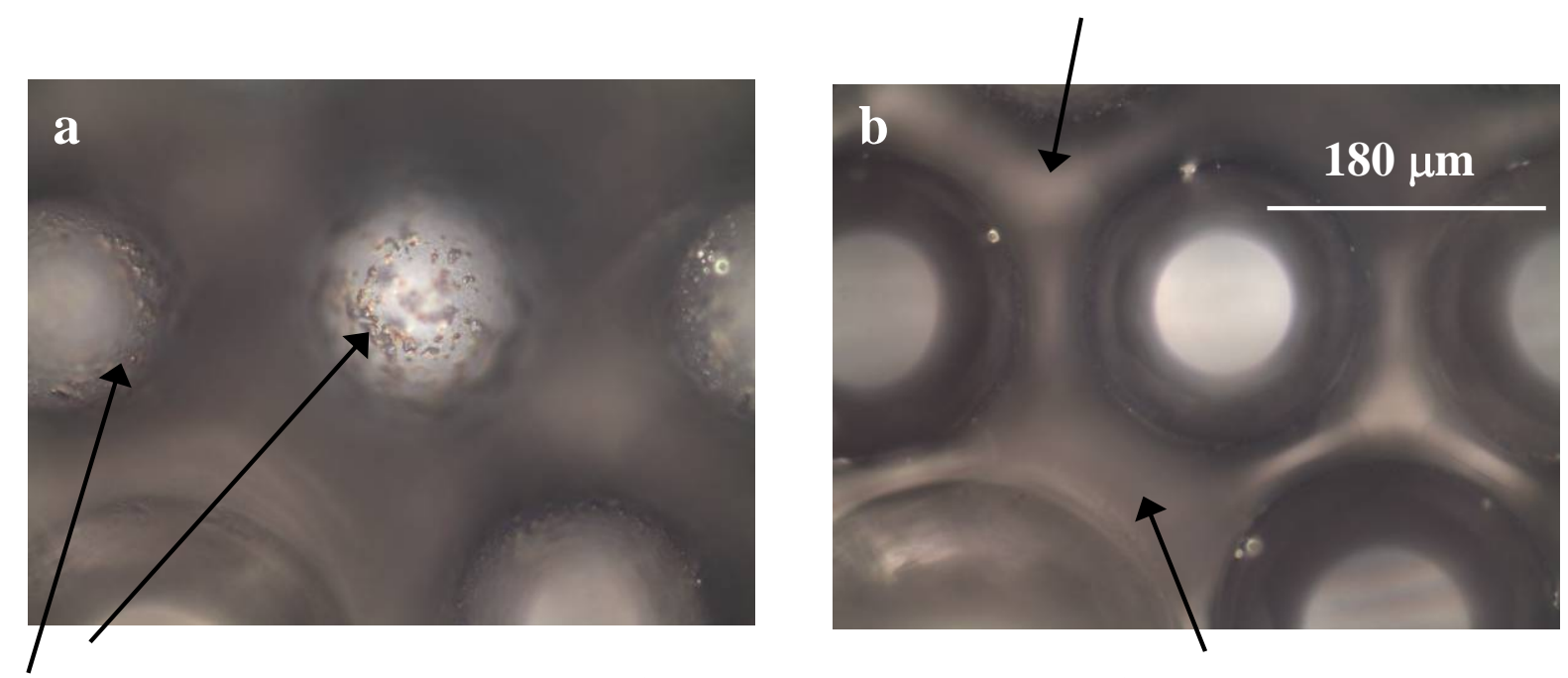

Figure 5.18a and 5.18b: $180 \mu \mathrm{m}$ beads from bead pack 9 at 500X magnification. Note the grainy coating of biofilm in 5.18a. The gel-like biofilm in 5.18b extends out from the bead at least twice its diameter as shown with a different focal plane than in "a".

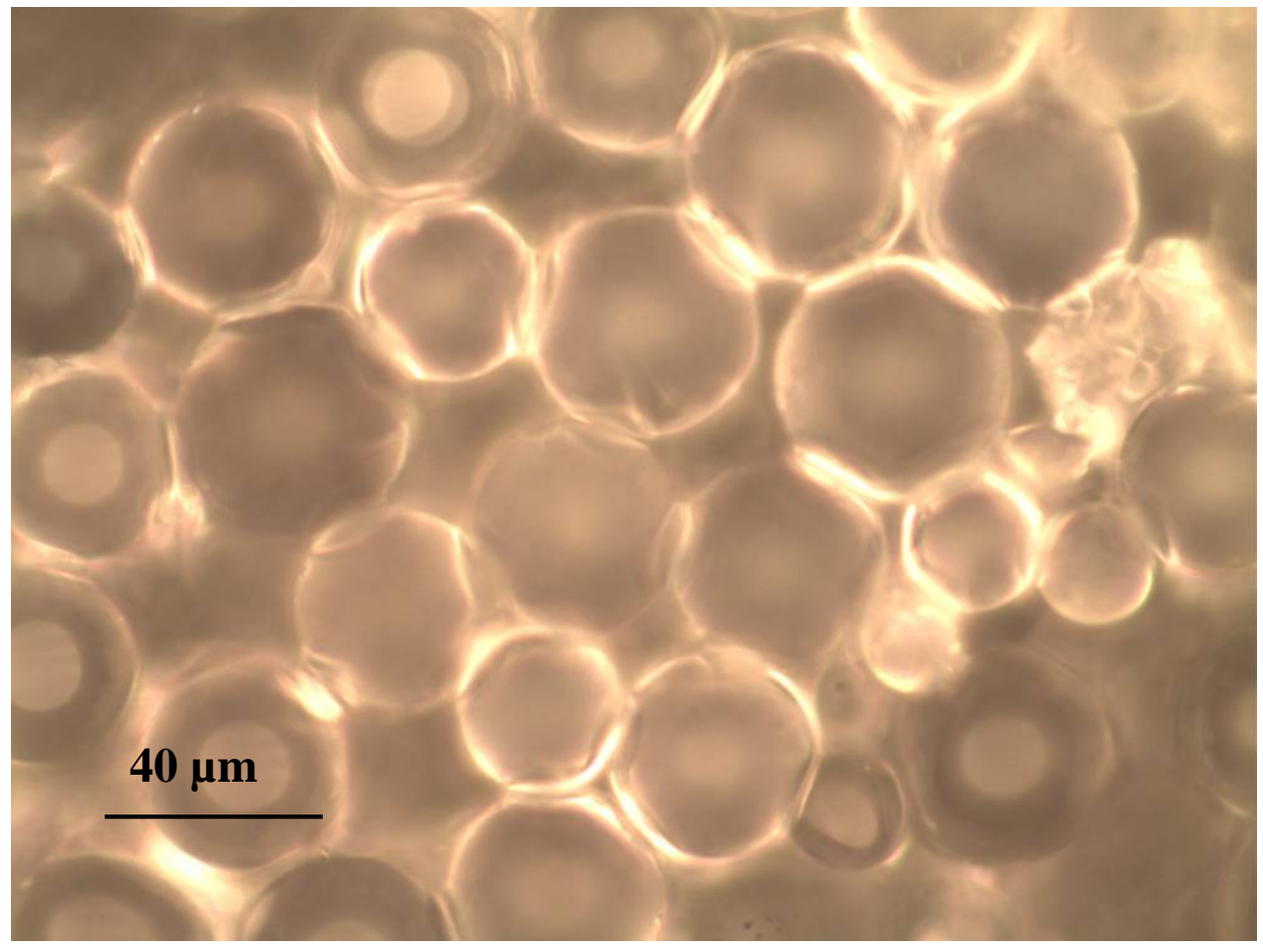

Figure 5.19: 500X magnification of $40 \mu \mathrm{m}$ beads shows lack of microbial growth 
Topic 1: Experimental evaluation of growth

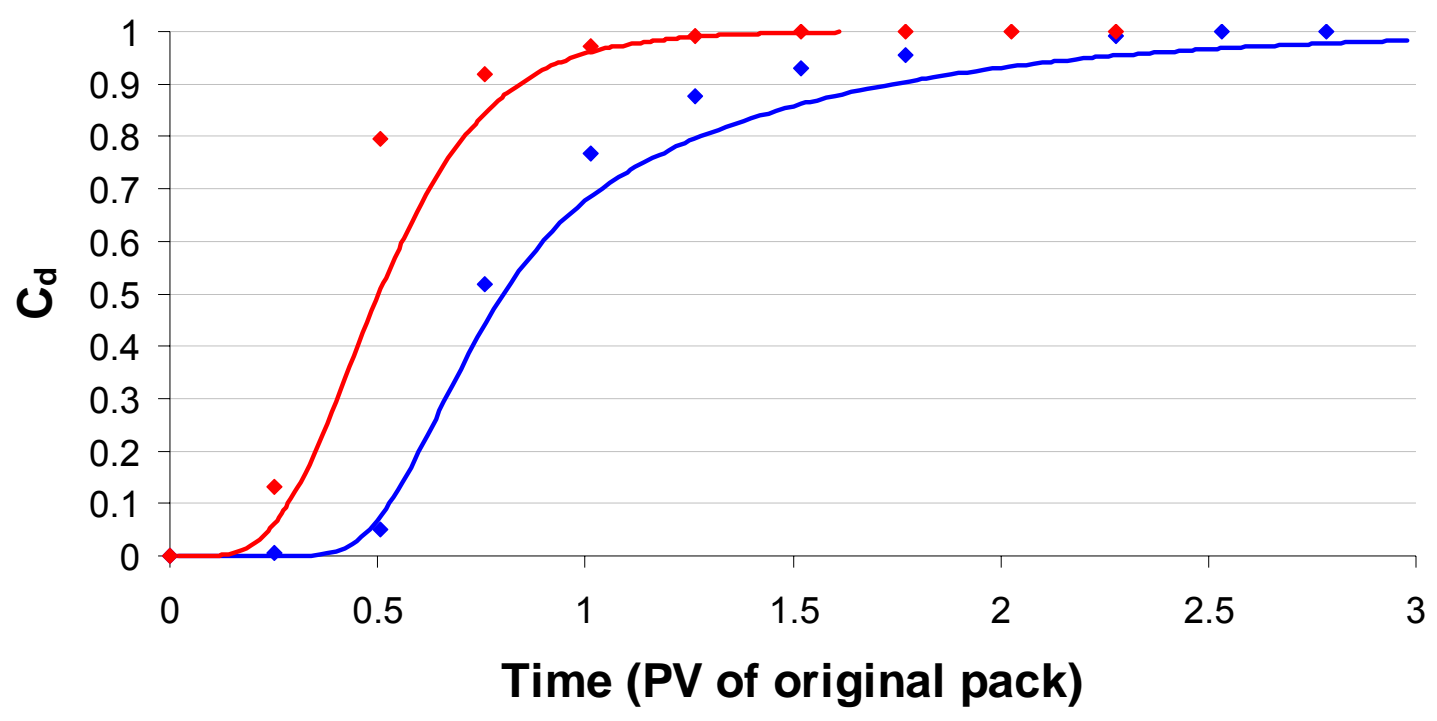

- pre-measured — pre-simulated $\bullet$ post-measured — post-simulated

Figure 5.20: UTCHEM pre and post biofilm history match for bead pack 9 


\section{Topic 1: COMPLETE CATALOG OF EXPERIMENTS -- Berea Core}

Two experiments with Berea cores were run to investigate biofilm induced permeability reduction in a commonly used sedimentary rock. The experimental procedures were the same as for the bead packs discussed in previous chapter.

\section{Berea Core A}

Berea core "A" had an overall permeability reduction of $72 \%\left(\mathrm{~K} / \mathrm{K}_{\mathrm{o}}=0.28\right.$; Figure 6.1). The majority of the permeability reduction was achieved within $6 \mathrm{PV}$ after which the $\mathrm{K} / \mathrm{K}_{\mathrm{o}}$ values varied between 0.22 and 0.34 (66-78\% reduction). Acetate concentrations from this core were measured only sporadically. Therefore, no overall conclusions on acetate consumption can be made.



- permeability - acetate

Figure 6.1: Permeability reduction and acetate utilization for Berea core A.

\section{Berea Core B}

Berea core " $\mathrm{B}$ " had an overall permeability reduction of $45 \%(\mathrm{~K} / \mathrm{Ko}=0.55$; Figure 6.2). The total permeability reduction was reached after $53 \mathrm{PV}$ and remained constant thereafter. Compared to the bead pack columns and Berea core " $\mathrm{A}$ " the permeability 
reduction in Berea core "B" was much slower. Effluent acetate concentrations decreased until $20 \mathrm{PV}$, then began to increase and reached a concentration of $94 \%$ of the injected acetate concentration at the end of the experiment (65 PV).

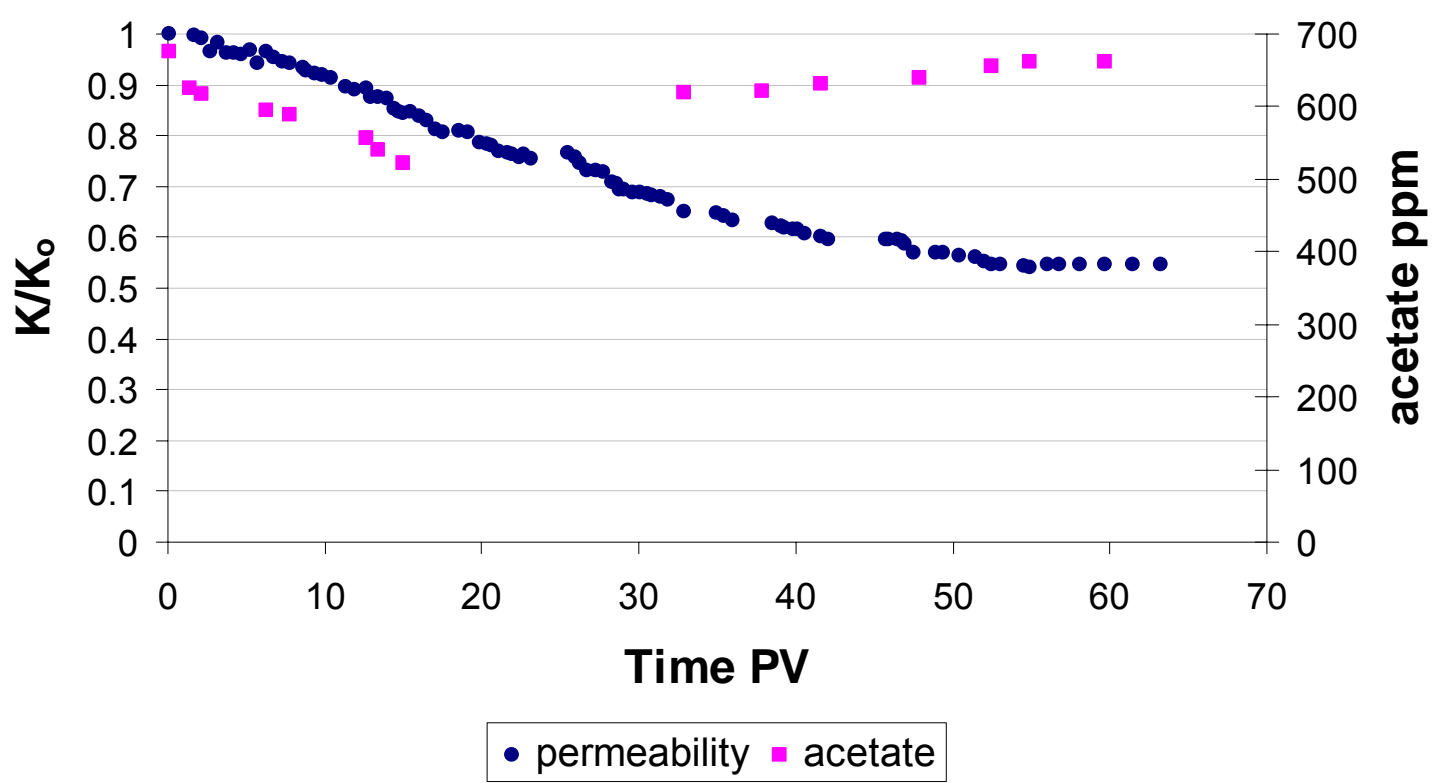

Figure 6.2: Permeability reduction and acetate utilization for Berea core B.

Berea core "A" was imaged using the high-resolution CT scanning before and after the flow experiment. Pre and post scans of the core were indistinguishable (Figure 6.3a and $6.3 \mathrm{~b}$ ). These images show that uranyl acetate staining of cells was unsuccessful possibly due to loss of biofilm during the staining process. 

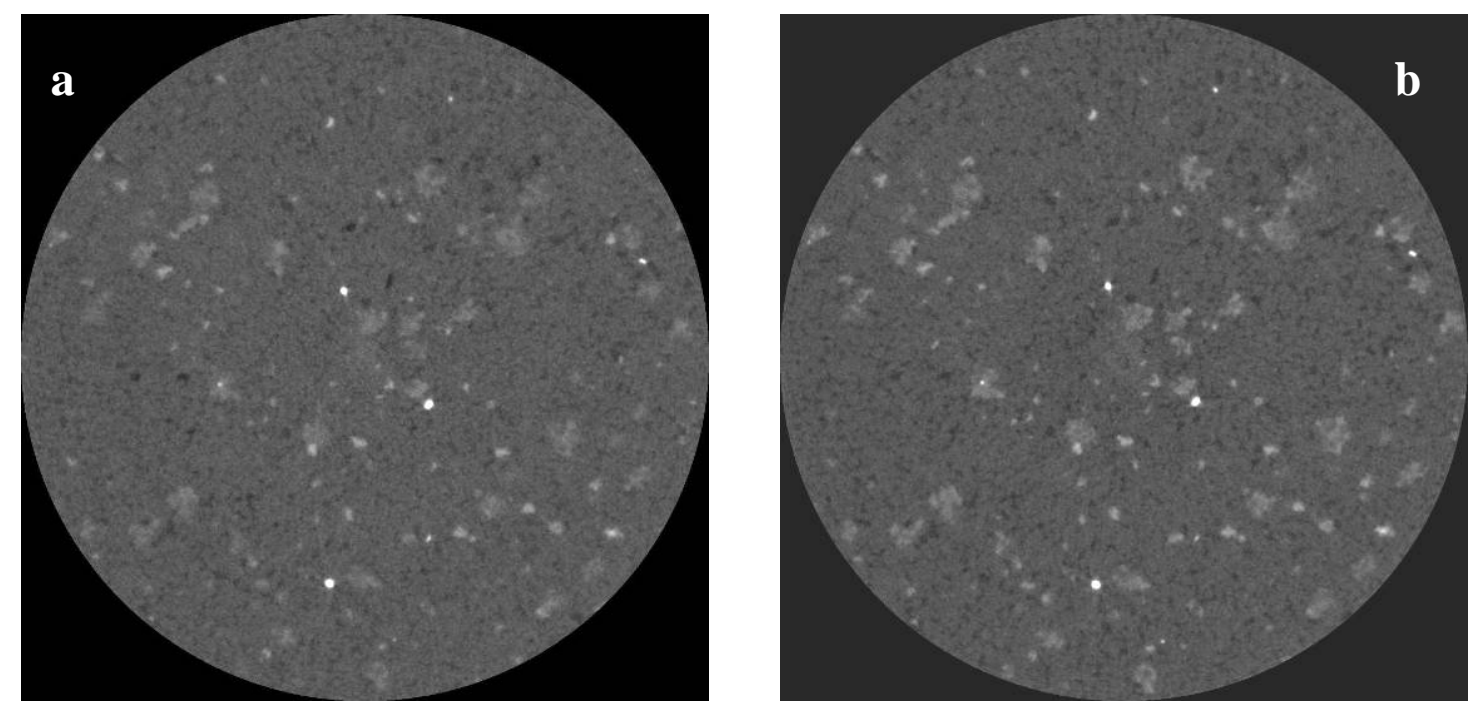

Figure 6.3a and 6.3b: Pre (a) and Post (b) experiment scanning stained with uranyl acetate. Dark areas indicate pores and other low density materials. Light areas indicate denser materials. There is no obvious difference between the two images. Either the biofilm was displaced from the core during the staining process (see text), or the staining was unsuccessful. 


\section{Topic 2: Modeling of Microbe Growth and Permeability Reduction}

\section{Summary}

Stimulating growth of indigenous microbes is a low cost method for improving sweep efficiency in water flooded reservoirs. Because injected nutrients for bacterial growth and biofilm development automatically enter thief zones, permeability reduction by accumulated biomass can channel the subsequently injected water into low permeability areas and thus increase sweep efficiency and the recovery factors.

We previously reported biofilm growth that preferentially reduced permeability of a high-permeability layer within a laboratory column. Here we show that traditional models of microbial behaviour in batch experiments do not explain the behaviour of the same microbes in flow experiments. Moreover, a standard model of the microbe behaviour during flow in a homogeneous porous medium cannot predict the behaviour in a heterogeneous medium. We discuss the present situation in understanding the growth of bacteria, nutrient consumption trends and their modeling in batch experiments. This paper also presents the possibility that quorum sensing is involved in the heterogeneous bead packs. We consider the capabilities of two simulators of coupled flow and reactive transport to account for experimental observations.

Applications of MEOR techniques require accurate modeling of experiments before field application. This section analyzes previous mechanistic models and experiments and outlines a modeling approach based on architecture and behaviour of biofilms. Such a model would account for the changing trend of nutrient consumption by bacteria with the increasing biomass and the onset of biofilm formation.

\section{Introduction}

Increased energy demands dictate the use of new and innovative EOR techniques that can increase recovery factors from reservoirs. Water flooding of maturing reservoirs is a preferred secondary recovery method. Reservoir heterogeneity plays a major role in the effectiveness of waterflooding. A stratified reservoir with significant permeability differences between the layers will have a faster breakthrough time and lower recoveries as the water preferentially channels into the higher permeability layers. These layers are often referred to as thief zones. Preferential channeling into certain layers over others decreases the sweep efficiency, a crucial factor in the overall recovery efficiency of a water flood. The MEOR method studied here proposes stimulating the growth of indigenous microbes. Selective microbial plugging of high permeability zones in a reservoir can lead to higher sweep efficiencies by diverting the flow of water from higher to lower permeability zones. However it is not only important to analyze increased sweep efficiencies but also the mechanisms by which biofilm growth occurs in the reservoir and the extent of reduction in permeability caused by growth.

Examples of stimulating microbe growth to improve sweep efficiency include the Blowhorn Creek oil unit located in the Black Warrior basin in Lamar County, Alabama. As discussed by Brown and Vadie (2000) production was enhanced and field life was 
also considerably extended. MEOR proved to be environmentally friendly and relatively inexpensive. Fuyu field in China is also a good example of this type of microbial process. High permeability zones were plugged by microbially-produced insoluble polymer to reduce water channeling (Nagase et al., 2001). This led to improved sweep efficiency. The Eunice Monument field in New Mexico is another example of a successful MEOR project (Gullapalli, 2000).

To apply this technology more widely it is important to understand the quantitative relationship between nutrient consumption, microbial growth and permeability reduction. In this paper we examine this relationship in a model system. Batch experiments were run with a Pseudomonas aeruginosa culture. These tests were used to calculate the various parameters in the Monod kinetics expression. These parameters were used successfully to model other batch experiments. Chemostat experiments were also run and modeled using these parameters. Flow experiments in various porous media were then simulated using the parameters from the batch experiments. The flow simulations did not match the observations, leading us to explore alternative ways of characterizing the process.

\section{Experimental Evidence for Permeability Reduction and Stimulating Microbe Growth}

Cunningham and Characklis (1991) injected various sand and glass bead columns with Pseudomonas aeruginosa. The permeability of these columns varied from 98 and 2127 Darcy. The columns were inoculated and shut in for 8 hours. Afterwards a glucose substrate was fed through the column with samples collected every 12 hours and the permeability calculated using the pressure difference across the column. Large permeability reductions occurred over a $2-3$ day period and smaller reductions over a 5 day period. The permeability reductions were due to growth of biofilm. The permeability reductions were in the range of $92-98 \%$. The coarser grained columns had higher reductions than the finer grained beads.

Chang et al. (1991) also explored biofilm growth in one-dimensional laboratory core floods. They incorporated terms for dispersion, convection, growth and decay. The growth in the core was modeled using Monod kinetics. They concluded that mathematical models developed from laboratory core floods can be successfully used to determine field-scale effects. A three-phase, multi-species one-dimensional model was developed to predict MEOR changes with two growth limiting substrates (Zhang et al, 1992). The Monod equation with two substrates was used to model the experiment. The model was able to predict phenomena like substrate consumption, product growth and permeability decrease. The model was tested with MEOR core floods. The model was able to relate microbe growth and permeability reduction.

Vandevivere and Baveye (1992) carried out experiments in sand packs to show difference in the permeability reduction capacities of microbes. The sand packs had porosities of about $39 \%$. They conducted experiments with the Arthrobacter sp. strain AK19 and the $\mathrm{SLI}^{-}$microbe. The AK 19 microbes produced permeability reductions of about $99 \%$ with about $98 \%$ of the biomass retained in the pack. These reductions occurred within 10 days. The $\mathrm{SLI}^{-}$microbes produced permeability reductions of less than $50 \%$ between 10 and 20 days. Only about $40 \%$ of the biomass created by this 
microbe was retained in the sand pack. These experiments clearly linked the permeability reduction to the amount of biomass retained in the core.

M.R. Islam (1994) used kinetic parameters, in addition to flow equations, to model microbial growth. He postulated that microbial growth would lead to either permeability reduction by pore plugging or alteration of oil properties such as reduction of viscosity or interfacial tension (IFT). Pore plugging was assumed to be dependent on multiplication of bacteria and generation of polymer. Each of these was modeled separately. The IFT reduction was modeled as a function of bacteria, and viscosity reduction was not modeled. The pore plugging model was shown to have scale-up problems and there was not enough experimental data to verify the accuracy of the IFT reduction model.

Lee and Bae (1998) examined the effects of salt concentration and $\mathrm{pH}$ changes on the Salton-1 microbe in Berea cores. They found that permeability reduction for this microbe was favored with a salt concentration of $2 \%$. Also there was more permeability reduction in basic and neutral environments than in acidic environments. The experiments also showed that nutrient concentration controlled biofilm growth and that produced biofilm was stable for long periods of time.

To obtain kinetic and stoichiometric data for Pseudomonas aeruginosa, Gandler et al. (2006) carried out a series of batch experiments. Samples were collected every four hours with two samples taken at each collection point. The samples were analyzed for acetate concentration and microbial concentration. Regression analysis was used to obtain an average trend for the microbial growth and acetate consumption.

Gandler et al. (2006) then conducted flow experiments in which the same strain was inoculated into cores and bead packs. Permeability was monitored as nutrient was continuously injected into the columns. All the bead packs were injected with $700 \mathrm{ppm}$ acetate. In all the experiments, the acetate monitored decreased to about $500 \mathrm{ppm}$ initially as the microbes utilized large amounts of nutrient for exponential growth. At the same time, large decreases in permeability occurred. At about $6-10 \mathrm{PV}$, the acetate levels in the effluent increased to about $650 \mathrm{ppm}$ and the permeability stayed constant. Evidently during the latter stages of the flow experiments, acetate was being utilized only for nutrition of the existing cells, not for growth.

Tracer tests showed effective porosity reductions of about $20 \%$ and average permeability reductions of about $60 \%$. The average permeability decrease in nine core experiments was $54 \%$ in a mean time of $6 \mathrm{PV}$. The experiments also clearly showed that the biofilm was able to divert flow from high permeability thief zones to lower permeability regions. Heterogeneous bead packs composed with various arrangements of coarse and fine beads were used for core flooding experiments. The arrangements were varied to create two layers with high and low permeability. The cores were then inoculated with bacterial culture and the substrate was injected continuously. Substrate concentration was measured in the effluent. Tracer tests before and after a successful biofilm growth experiment in a dual layered bead pack showed that the permeability of the coarse bead layer was reduced by a factor of 20 after biofilm growth, while the fine bead layer experienced no significant reduction in permeability because no growth occurred in that layer. 
In summary, batch experiments are instrumental in studying the growth and substrate uptake behavior of microbes. They are standard for obtaining kinetics expressions. Core flooding experiments give insight into how microbes grow in the much more confined space of voids in a porous medium. In particular the adaptive response of forming biofilms appears to occur differently in pores than in batch. Tracer experiments in corefloods are a simple but valuable method of inferring the distribution of microbial growth.

\section{Modeling Data with Classical Approach (Monod Kinetics)}

We illustrate the kinetic model development using batch experiments in which Pseudomonas aeruginosa PAO-1 microbial cultures were placed in Erlenmeyer flasks in an oxygen rich sterile environment (Gbosi, 2007).

The growth curves (Fig. 1) followed the general trend of classical growth curves of biological species, with a lag phase, a period of exponential growth and then a stationary (mature) phase. From the first set of batch experiments, a least squares fit was done to obtain values for these Monod parameters. Least-squares best fit curves were fit to the acetate and microbial data so the Monod parameters could be calculated. Monod equation can be simply described as follows

$$
\mu=\mu_{\max } \mathrm{S} /\left(\mathrm{S}+\mathrm{K}_{\mathrm{s}}\right)
$$

where $\mu=$ specific growth rate $\left(\mathrm{hr}^{-1}\right), \mathrm{S}=$ Substrate concentration (ppm), $\mathrm{K}_{\mathrm{s}}=$ half saturation constant (ppm), and $\mu_{\max }=$ maximum specific growth rate $\left(\mathrm{hr}^{-1}\right)$.

The specific growth rate is defined as the rate of growth per unit amount of biomass. The half saturation constant is the substrate concentration at exactly half of the maximum specific growth rate.

According to Monod kinetics, the substrate reaction rate in a bacterial culture is as follows

$$
\mathrm{dC}_{\mathrm{L}} / \mathrm{dt}=-\mu_{\max } \mathrm{X}_{\mathrm{a}} \mathrm{C}_{\mathrm{L}} /\left(\mathrm{K}_{\mathrm{s}}+\mathrm{C}_{\mathrm{L}}\right)
$$

where $\mathrm{C}_{\mathrm{L}}$ is the substrate concentration in ppm and $\mathrm{X}_{\mathrm{a}}$ is the initial microbe concentration.

The active cell concentration with growth can be described by

$$
\mathrm{X}_{\mathrm{a}}=\mathrm{X}_{\mathrm{ao}}+\mathrm{Y}\left(\mathrm{C}_{\mathrm{LO}}-\mathrm{C}_{\mathrm{L}}\right)
$$

where $\mathrm{Y}$ is the yield coefficient in colony-forming units (cfu) per milligram of acetate and $\mathrm{C}_{\mathrm{LO}}$ is the initial acetate concentration that is introduced in the batch culture. The value for $\mathrm{Y}$ determined from the batch experimental data was $3.2 \times 10^{9} \mathrm{cfu} / \mathrm{mg}$-acetate, for $\mathrm{X}_{\mathrm{ao}} 4.5 \times 10^{6} \mathrm{cfu} / \mathrm{ml}$, for $\mathrm{K}_{\mathrm{s}} 450 \mathrm{ppm}$ and for $\mu_{\max } 0.35 \mathrm{hr}^{-1}$.

The growth rate is a function of initial microbe concentration and the measured acetate concentration at various times in the batch experiments. Experimental data from the 700 ppm batch culture is compared to predictions from Eqs. 1 and 2 using the parameters mentioned above in Figs. 2 and 3. The bacterial growth modeled by this equation followed closely the best fit experimental curve with differences at the start and at the end of the experimentation. The initial values were higher than the best fit experimental curve while slightly lower in the last few hours. Similarly, the nutrient uptake history as 
predicted by the equation for substrate uptake (Fig. 3) matched the best fit experimental curve and showed some difference in the values at a later time. The experimental data, the best fit curve and the modeled substrate uptake show reasonable agreement.

For the flow experiments silica glass beads were used to create columns as an alternative porous medium to the Berea cores. Using the glass beads proved advantageous because different porosity and permeability configurations could be created by varying the sizes and locations of beads in the column, Fig. 4. Both homogeneous and heterogeneous packs were used. A typical substrate and permeability history in such a column is shown in Fig. 5.

The flow experiments were simulated with UTCHEM. UTCHEM has a biodegradation option used to model biological kinetics. This option was added to model groundwater cleanup (Delshad et al, 2002). The Monod parameters from the batch experiments were input into the simulator and the porosity and permeability reduction option was enabled. With UTCHEM it was possible to simulate tracer injection but reasonable results for microbial growth could not be obtained.

The flow experiments were then simulated with the reactive transport simulator PARSSIM (Arbogast et al, 1998), selected because of its capacity to simulate biological growth with general Monod kinetics and stationary microbes. The approach taken was to treat the bead pack as a 1-D domain. In the simulation, the initial condition set the microbes to be attached to the surface of the beads at a uniform concentration throughout the pack. The acetate was assumed to be in the flowing phase. The parameters obtained from the batch experiments were input as values for the Monod equation in the simulator.

The history of substrate concentration and microbes were taken at the last grid block of the simulation grid. The results are shown in Fig. 6. However, the growth of the microbes and consequent consumption of the acetate occurred almost instantaneously instead of gradually as was expected. A gradual transition was expected because growth in the batch experiments occurred over $40-60$ hours and the mean residence time of acetate in the cores was 3.5 hours. To check the qualitative validity of the simulation, the reaction rate was slowed by reducing $\mu_{\max }$. The resulting concentration of acetate in the last grid block of the simulation grid is shown in Fig. 7. The acetate history as predicted by PARSSIM showed that the acetate was consumed immediately instead of being gradually used up. With even slower reaction rate as shown in Fig. 7 the results were qualitatively the same as when the simulation was run at faster reaction rate (Fig. 6). The implications of these observations are discussed in the next section.

The tracer simulations for heterogeneous bead packs were done with UTCHEM, a multiphase, multi-component reservoir simulator. Tracer experiments in the bead packs were carried out to provide indirect evidence (via the effluent history) of flow patterns within the pack. Adjusting the distribution of porosity, permeability and dispersivity within the simulator enabled a match of the observed effluent history and revealed the likely distribution of microbes after nutrient injection. Figures 8 and 9 illustrate the preference of flow in high permeability layers before nutrient injection, and the subsequent diversion of flow into low permeability layers caused by biofilm growth. 


\section{Discussion of Bead Pack Experiments and Model}

Using Monod kinetics that fit the batch experiments, the simulator predicted different behavior than the experiments. Changing Monod model parameters had no effect on the outcome (Fig. 7). It was evident that the core flood experiments could not be predicted by changing Monod kinetics parameters. The physical reason is simple: Monod batch kinetics does not predict a limiting concentration of microbes. Rather, a microbe population following Eq. 1 will grow without bound as long as nutrient is available. In a flow experiment, a fresh supply of nutrient is made available continuously. Thus any reactive transport simulator using Monod kinetics will necessarily predict increasing concentration of microbes and the corresponding increased consumption of the substrate. So soon after simulated nutrient injection begins, the increased microbe concentration consumes all the injected acetate long before it can reach the end of the domain.

How can the discrepancy between batch and flow behavior be reconciled? One possibility is that biofilm forms within the porous media, but not in the batch experiments. Biofilms are self-organized microbial communities arranged by stacking cells. In many surface environments a biofilm offers survival advantages to the community as a whole, though cells on the outer layer are less likely to survive events such as drying, irradiation or feeding by other organisms. It is not yet clear whether advantages are inherent for biofilms within a porous medium. In any case, it is likely that the lower stack of cells exhibits different nutrient consumption kinetics compared to the upper stack of cells. One reason is the decreased availability of nutrients. The concentration of the nutrients decreases from the surface of the biofilm to the inner stacks of cells because the flux through the film must be driven by a concentration gradient. Secondly, the innermost cells may well exhibit a different intrinsic metabolic rate than the outer stack of cells.

Another reason for the inability of Monod kinetics to predict the acetate history in bead packs might be quorum sensing. Quorum sensing plays a role in controlling the growth kinetics of the biofilm in surface environments (Ward, 2001). This ability signals the microbes to start the creation of the biofilm when the population density is high enough. Quorum sensing is a cell-to-cell communication phenomenon. In effect the bacterial cells seem to monitor their own population density and regulate their behavior in a multicellular fashion as the cell density changes. Thus, as the population increases and reaches a certain level a phenotypic change may be induced which would not be worthwhile for individual cells surviving alone. This density dependent behavior alters the growth rate and the substrate uptake in biofilms.

The process of quorum sensing is regulated by the production and concentrations of quorum sensing chemicals produced by bacteria. A quorum sensing model would treat that growth of population of bacteria consisting of cells as either up regulated or down regulated, the former producing quorum sensing molecules in larger amounts to down size the bacterial growth. Docker and Keener (2001) have modeled the quorum sensing process in $P$. Aeruginosa and have explained this phenomenon in detail. After maturity, high concentration of quorum sensing chemicals in the biofilm restricts further growth or multiplication of cells. Thus the biofilm only consumes nutrients for essential metabolic processes. This would be consistent with the observed effluent history, i.e. steady 
concentration of acetate slightly below the injected value. The traditional Monod kinetics of Eq. 1 has no term corresponding to the concentration of quorum sensing chemical or the "switch" activated by this chemical to stop cell multiplication.

Yet another reason for Monod kinetics not predicting the acetate history in the flow experiment is that it does not explicitly account for the phenomena responsible for acetate transport within a biofilm. The transport of substrate in moving liquid is governed by molecular diffusion, hydrodynamic dispersion and convection. Substrate transport rate through the biofilm is dependent on the substrate diffusivity through the biofilm. Diffusion of substrate into the biofilm structure continues after the biofilm has reached maturity. The nutrients may later be consumed for various metabolic processes or they may remain in the biofilm for some time before being consumed. If the biofilm reaches a steady-state configuration, all the nutrient that diffuses into the film will be consumed, and the diffusive flux will become constant. This would lead to a steady effluent concentration of the substrate somewhat below the injected value.

Oxygen may also act as a limiting agent inhibiting the growth of microbes. Conventionally this is accounted for in Monod kinetics by regarding oxygen as an electron acceptor. This introduces a new term that is similar to the substrate term in Equation 1:

$$
\mu=\mu_{\max } \mathrm{S} /\left(\mathrm{S}+\mathrm{K}_{\mathrm{s}}\right) \times \mathrm{S}_{\mathrm{o}} /\left(\mathrm{S}_{\mathrm{o}}+\mathrm{K}_{\mathrm{os}}\right)
$$

where $\mathrm{S}_{\mathrm{o}}$ is the oxygen concentration and $\mathrm{K}_{\mathrm{os}}$ is the half saturation constant for oxygen in appropriate units. In the batch systems, oxygen was in excess, whereas in the packed bed experiments, oxygen availability may become limited before the nutrient reached the end of the column. Restricted oxygen supply reduces the growth rate of bacteria and may cause them to become dormant. This limited supply of oxygen leads to lower metabolic rate in the cells. This might explain the steady state concentration of acetate slightly below the injected value, when the biofilm has reached maturity.

In the case of heterogeneous bead packs the flow diversion due to biofilm was confirmed with help of tracer experiments and biofilm imaging. The effluent acetate history in the case of heterogeneous bead packs was qualitatively similar to the homogenous (Fig. 10) bead pack experiments. But in the case of heterogeneous bead packs, a lower minimum of acetate concentration reflected larger consumption rates (Fig. 11). Tracer experiments showed significant biofilm formation and growth in the coarse beads where most the flow passes through initially. High flow rate in coarser beads means a higher supply of nutrients. However it also means less residence time for reaction to occur. Moreover the Monod reaction rate depends only on concentrations, not on nutrient fluxes, and the concentrations entering coarse and fine bead layers are the same. Surface area available for the biofilms to adsorb also varies. The surface area available for adsorption is larger in case of finer beads as compared to that in coarser beads, but still biofilm formed preferentially in the coarser beads. Other factors discussed above that may affect growth remain the same for homogeneous and heterogeneous bead packs. Thus modified growth kinetics models that are able to predict the acetate consumption in homogeneous packs will not predict the observation that biofilms grew preferentially in the coarser beads or the high permeability areas. 


\section{Alternative Model}

The simulations illustrate that Monod kinetics describes batch experiments, but not microbial growth in flow experiments in porous media. In batch experiments the kinetics model accounts for observations, since all the cells have equal access to nutrient. In porous media, the lack of access to nutrient for some of the cells and the biofilm itself seems to change the microbial kinetics. The flow experiments are qualitatively consistent with a kinetics that decreases the microbial specific growth rate with time, even though substrate (acetate) remains available in large concentrations. We therefore seek a model that accounts for microbial growth and yet allows a decreasing microbial specific growth rate.

Typically a biofilm is considered to be uniform in simple modeling efforts. Factors such as biofilm depth and its effect on nutrient uptake are neglected. Here we also ignore diffusion within the biofilm (Fig. 12). Diffusion and the other phenomenon of mass transport in porous media become dominant after a considerable period of time when the biofilm has reached maturity. Nutrient concentration gradients exist in large biofilms and they alter the diffusion or uptake of substrate at the surface of the biofilm (Bishop and Rittmann, 1995).

Ignoring these mass-transport steps, we focus instead on the effect of altering the "driving force" term in the kinetics expression. The metabolic processes of microorganisms are inhibited by certain nutrients at sufficiently higher concentrations. Thus, the growth of the micro organisms is inhibited at higher concentrations resulting in lower nutrient uptake for growth, Fig. 13. This behavior would be consistent with the increasing concentration of substrate in the effluent at later times during the flow experiments. There is a critical concentration of acetate above which the specific growth rate of the microorganism decreases with increasing substrate concentration (Sivakumar, 1994). Andrews and Noack model accounts for substrate inhibition (Edwards, 1970) and was fitted to the available data.

The model can be written

$$
\mu=\mu_{\max } \mathrm{C}_{\mathrm{s}} /\left(\left(\mathrm{K}_{\mathrm{s}}+\mathrm{C}_{\mathrm{s}}\right)+\left(1+\mathrm{C}_{\mathrm{s}} / \mathrm{K}_{\mathrm{i}}\right)\right)
$$

The constant $\mathrm{K}_{\mathrm{i}}$ describes the inhibition coefficient while the other terms remain the same as that explained in the Monod kinetics equation.

We apply Eq. 5 with the same values of the Monod parameters as used in the simple Monod kinetics equation (Eq. 1). The values of inhibition constant $\mathrm{K}_{\mathrm{i}}$ were varied. Large values have less effect on the specific growth rate; small values increase the inhibition. Mathematically this is due to the term $\left(1+\mathrm{C}_{\mathrm{s}} / \mathrm{K}_{\mathrm{i}}\right)$ in the denominator in equation (5); the effect of this term on the equation diminishes as $\mathrm{K}_{\mathrm{i}}$ increases. During an experiment, the effect of the term $\mathrm{C}_{\mathrm{s}} / \mathrm{K}_{\mathrm{i}}$ increases as time increases and the substrate concentration in the effluent increases; however the magnitude may not be large enough to affect the final value of the specific growth rate. Thus a large inhibition constant would allow a small change in the specific growth rate despite the increasing substrate concentration.

The typical behavior in substrate inhibited experiments (Fig. 13) is that the specific growth rate increases and reaches a maximum value and then it shows a decline in the 
specific growth rate. At suitable values for inhibition constants such as those of 100,300 and $400 \mathrm{ppm}$, the growth curve (Fig. 14) shows the behavior typical of substrate-inhibited microbial growth. Therefore inhibition may also be thought of as a possible factor that might predict the decreasing microbial growth and increasing substrate concentrations in the effluent in case of bead pack experiments. Continuous injection of a certain substrate concentration leads to greater exposure time of biofilms to the substrate. Therefore the effect of substrate inhibition may be pronounced in case of bead pack experiments as compared to that in batch experiments, where substrate is introduced only once in the microbe culture.

The analysis of an alternative self-limited growth model is given in the supplementary material at the end of this section.

\section{Conclusions}

Monod kinetics accounts for the simple microbial growth and substrate uptake in batch experiments with the model organism P. Aeruginosa. However it cannot account for substrate uptake in nutrient injection experiments in porous media inoculated with the same organism. A substrate inhibition model describes the moderation of microbial growth rate with time. Decreased microbe growth rate may be able to explain the increasing acetate concentration in the effluent after a certain period of time. However for predictive modeling of the flow experiments, quorum sensing and diffusion through the biofilm are most likely to be the relevant phenomena missing from current kinetics models. Even after including these phenomena, however, it is not clear whether the resulting model will account for the preferential growth observed in coarse beads.

\section{References}

Arbogast, T: "User's Guide to PARSSIM 1: The parallel subsurface simulator, single phase,” May 1998.

Andrews, G: "Effectiveness Factor for Bioparticles with Monod Kinetics," Chemical Engineering Journal, issue 37, 1988.

Bishop, P.L and Rittmann, B.E: "Modeling Heterogeneity in Biofilms: report of discussion session, Water Science and Technology, Vol 32 (8), p 263-265, 1995.

Brown, L., Vadie, A: "Slowing Production Decline and Extending the Economic Life of an Oil Field: New MEOR Technology.SPE 75355, SPEREE, February 2002.

Brown, L.R: "Slowing Production Decline and Extending the Economic Life of an Oil Field: New MEOR Technology," SPE 59306 presented at the SPE/DOE Improved Oil Recovery Symposium, Tulsa, Oklahoma, 3-5 April 2007.

Bonomo, L "Simplified and Monod Kinetics in one-dimensional biofilm reactor modeling: a comparison," Water Science and Technology, Volume 43 No 1, pp 295-302, 2001.

Byl, Tom D: US Geological Survey Karst Interest Group Proceedings, Water investigations Report 01-4011, p.151$156,2001$.

Chang, M-M., Chung, F.T-H., Bryant, R.S., Gao, H.W., Burchfield, T.E.: "Modeling and Laboratory Investigation of Microbial Transport Phenomena in Porous Media," SPE 22845 presented at $66^{\text {th }}$ Annual Technical Conference and Exhibition, Dallas, Texas, 6-9 October, 1991.

Cunningham, A.B, : "Influence of Biofilm Accumulation Porous Media Hydrodynamics," Environ, Sci, Technol, issue $25,1305-1311,1991$.

Docker, J.D and Keener, J: “A Mathematical Model for Quorum Sensing in P. aeuroginosa,” Bull. Math. Biology., issue 63, p 95-116, 2001.

Delshad, Mojdeh: "Simulation of Chemical and Microbial Enhanced oil Recover methods," SPE 75237 presented at the SPE/DOE Improved Oil Recovery Symposium held in Tulsa, Oklahoma, 13-17 April, 2002.

Dietrich, F.L et al: "Microbial EOR Technology Advancement: Case studies of successful projects," SPE 36746 presented at the 1996 SPE Annual Technical Conference and Exhibition ,Denver, Colorado, 6-9 October, 1996.

Edwards, V. H.: Biotechnol. Bioeng, 12 (1970), 679. 
Gandler, G.L: "Mechanistic Understanding of Microbial Plugging for Improved Sweep Efficiency," SPE 100048 presented at SPE/DOE Symposium on Improved Oil Recovery, Tulsa, Oklahoma, 22-26 April, 2006.

Gbosi ,Akpobari : "Parameter Modeling for Mechanistic Understanding of Microbial Plugging," M.S Thesis ,University of Texas at Austin, 2007.

Gullapalli, I.L: “A Laboratory Design and Field Implementation of Microbial Profile Modification Process,” SPEREE, $42,2000$.

Hentzer, Morten: "Alignate Overproduction effects of Pseudomonas aeruginosa Biofilm Structure and Function," Journal of Bacteriology, Vol 183 no 18, p 5395-5401, Sept 2001.

Harvey, Ronald W: "Association of free living bacteria and dissolved organic compounds in a plume of contaminated ground water," Journal of Contaminant Hydrology, Issue 9, p 91-103, 1992.

Islam, M.R: "Mathematical Modeling of Microbial Enhanced Oil Recovery" SPE 24080 presented at the $65^{\text {th }}$ Annual Technical Conference and Exhibition, New Orleans, Louisiana, 23-26 September, 1990.

Jenneman, G.E: "SPE 10789 Experimental Studies of In-Situ Microbial Enhanced Oil Recovery." SPE Journal 24(1): 33-37.1984.

Lee, H.O, Bae, Jae H: "Effects of Variables on Microbial Profile Modification with Spores," presented at SPE/DOE Improved Oil Recovery Symposium, Tulsa, Oklahoma, 19-22 April 1998,

Maudgalya, S: "Microbial Enhanced Oil Recovery Technologies. A Review of the Past, Present and Future," SPE 106978-MS presented at The Production and Operations Symposium. Oklahoma City, Oklahoma, 31 March-3 April, 2007.

Nagase, K: "Improvement of Sweep Efficiency by Microbial EOR Process in Fuyu Oilfield, China," SPE 68720 presented at the SPE Asia Pacific Oil and Gas Conference and Exhibition held in Jakarta, Indonesia, 17-19 April, 2001.

Raiders, R.A: "The Use of Microorganisms to Increase the Recovery of Oil from Cores," SPE 14336-MS presented at The SPE Annual Technical Conference and Exhibition. Las Vegas, Nevada, 22-25 September, 1985.

Smith, Richard L: "Importance of closely packed vertical sampling in delineating chemical and microbiological gradients in groundwater studies," Journal of Contaminant Hydrology, Vol 7, p 285-300, 1991.

Stewart, T.L: "Modeling of biomass-plug development and propagation in porous media." Biochemical Engineering Journal 17(2): 107-119. (2004).

Sivakumar, A: "Extended Monod Kinetics for Substrate inhibited systems," Bioprocess Engineering, issue 11, p 185188, 1994.

Tufenkji, Nathalie: "Modeling microbial transport in porous media: Traditional approaches and recent developments," Advances in Water Resources, issue 30, p 1455-1469, 2007.

Taylor, S. W: "Enhanced in-situ biodegradation and aquifer permeability reduction." Journal of Environmental Engineering-ASCE 117(1): 25-46, 1991.

Taylor, S. W. and P. R. Jaff: "Biofilm Growth and the Related Changes in the Physical Properties of a Porous Medium Experimental Investigation." Water Resources Research 26(9): 2153-2159. (1990).

Thullner, M: "Modeling of a microbial growth experiment with bioclogging in a two-dimensional saturated porous media flow field," Journal of Contaminant Hydrology, 70(1-2): 37-62. (2004).

UTCHEM-.9.0, Vol. 2: Technical Documentation prepared Center for Petroleum and Geosystems Engineering, The University of Texas at Austin, 2000.

Van Loosdrecht: "Mathematical Modeling of Biofilm Structures," Antonie van Leeuwenhoek, Vol 81, p 245-256, 2002.

Vandevivere, P, Baveye, P: "Relationship between Transportof Bacteria and their Clogging Efficiency in Sand Columns," Applied and Environmental Microbiology, Ausgust, 1992.

Ward, J.P: "Mathematical Modeling of Quporum sensing in Bacteria,"Journal of Mathematics apllied in medicine and Biology, issue 18, 2001.

Zhang, T.C and Bishop, P.L: "Structure activity and composition of biofilms,"Water Sci Tech 29 (7), p 335-344.

Zhang, XU: "A Mathematical Model for Microbially Enhanced Oil Recovery Process," SPE/DOE 24202 presented at the eight symposium on Enhanced Oil Recovery, Tulsa, Oklahoma, 22-24 April, 1992. 


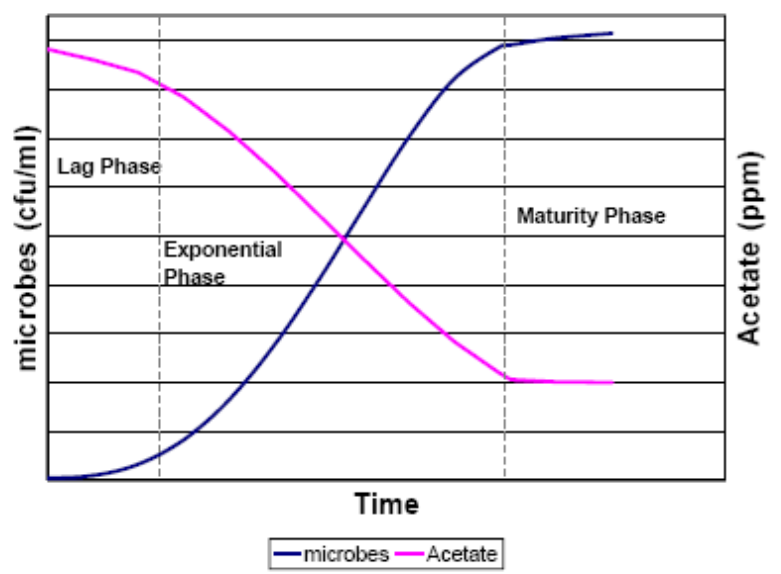

Fig. 1-Typical Monod kinetics curve for microbial growth and nutrient consumption in batch experiments. The above graph shows three phases of bacterial growth i.e lag, exponential and maturity phase.

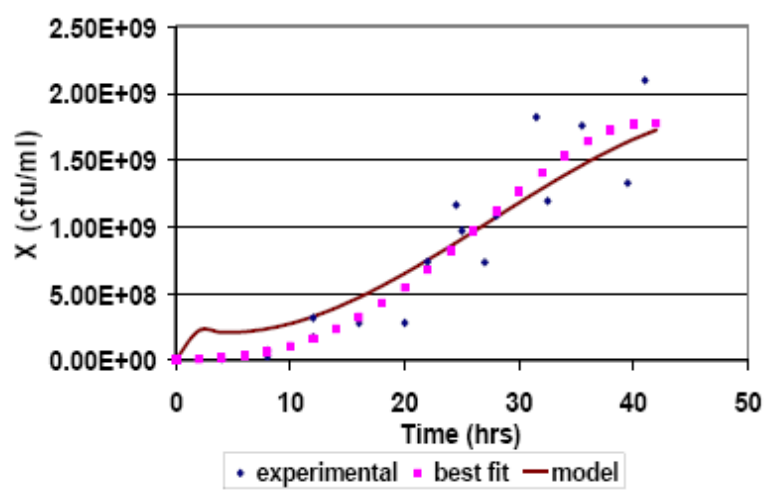

Fig. 2-Microbial growth curve for $700 \mathrm{ppm}$ batch culture. The Monod kinetics expression explains the general trend of the data.

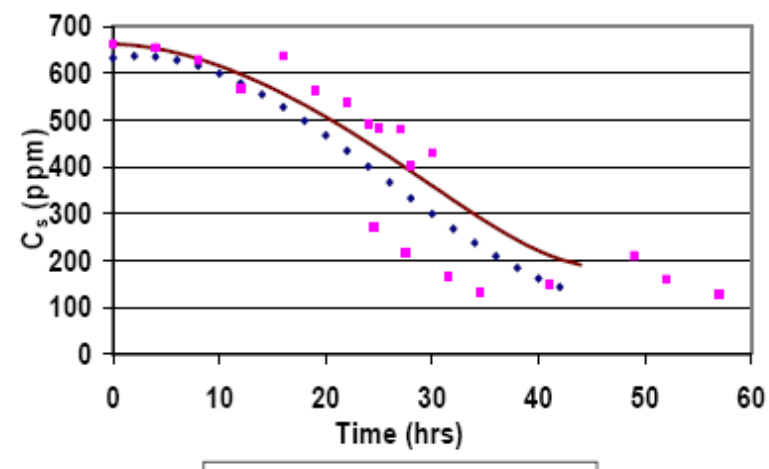

- best fit " experimental -model

Fig. 3-Acetate consumption trend for the $700 \mathrm{ppm}$ batch culture. The Monod expression explains the general trend of the data. 


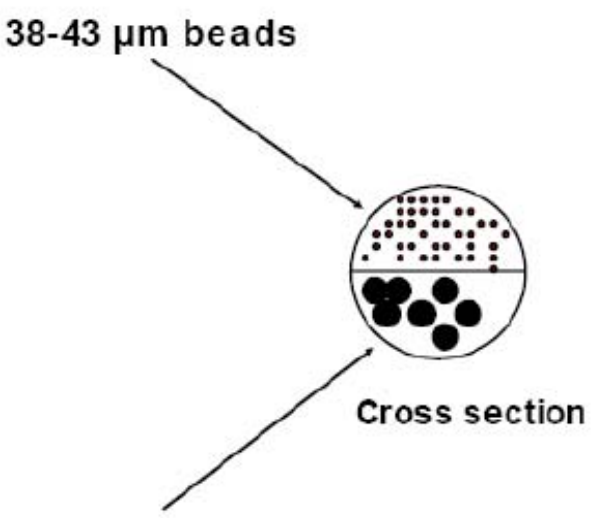

$180 \mu \mathrm{m}$ beads

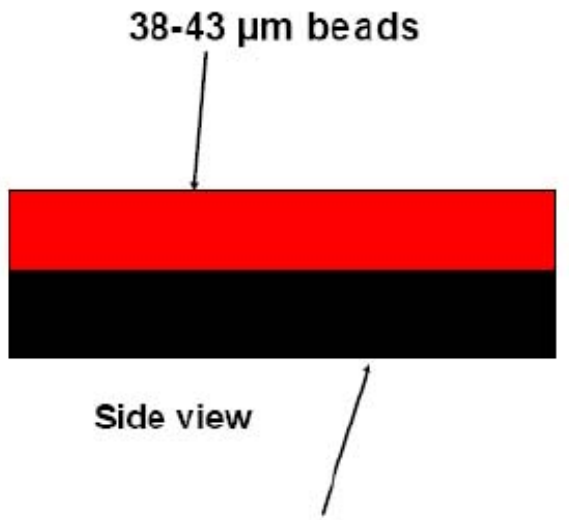

$180 \mu \mathrm{m}$ beads

Fig. 4-A schematic of the dual-layer heterogeneous bead pack experiment (adapted from Gandler et al. (2006)).

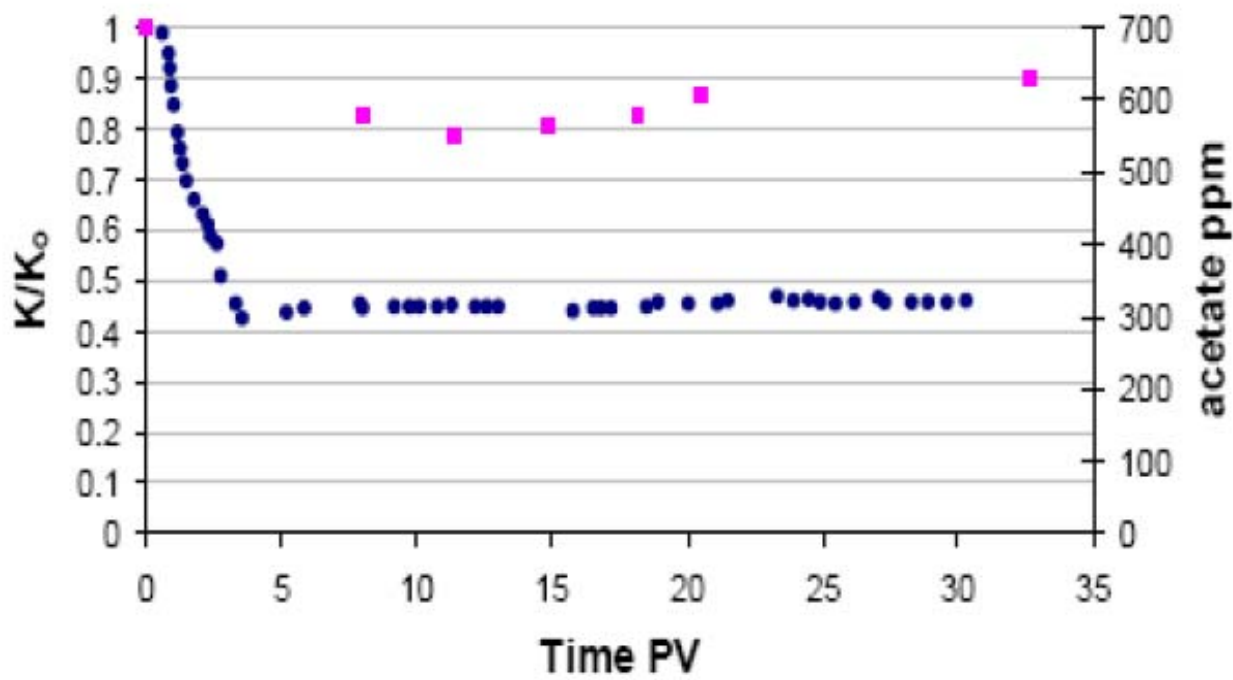

- permeability $\approx$ acetate

Fig. 5-Typical behavior during nutrient injection in a homogeneous bead back inoculated with $\boldsymbol{P}$. Aeruginosa (Gandler et al., 2006). 
Topic 2: Modeling of growth

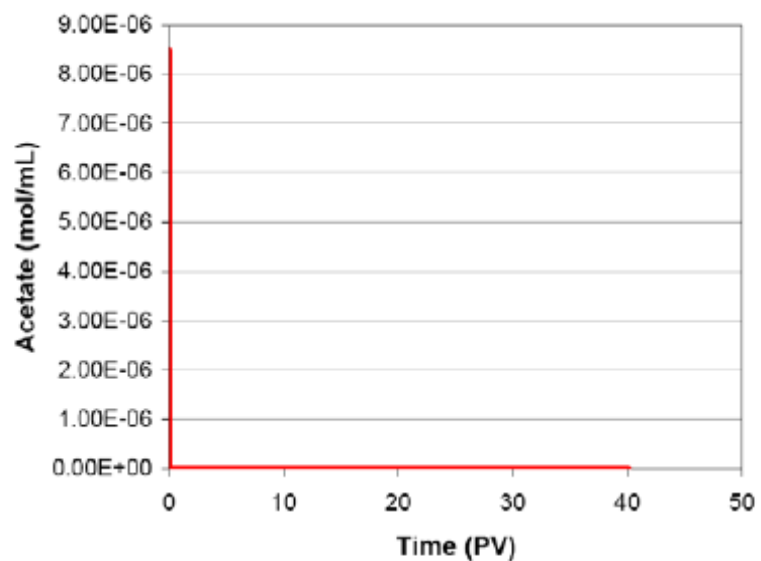

(a)

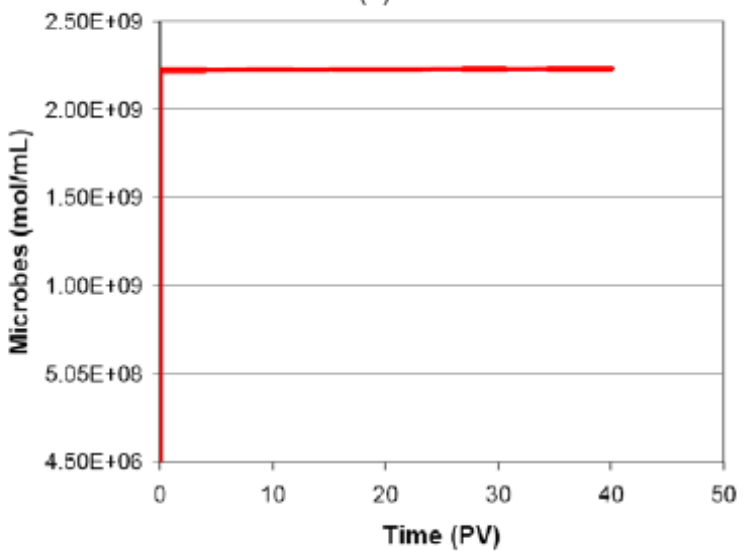

(b)

Fig. 6-(a) Acetate consumption profile at the last grid block of the simulation grid as predicted by PARSSIM. The simulation used Monod kinetics parameters from batch experiments (Figs. $2,3)$ for predicting acetate consumption in the core flood experiment. (b) Microbial concentration at the center of the last grid block as predicted by PARSSIM.

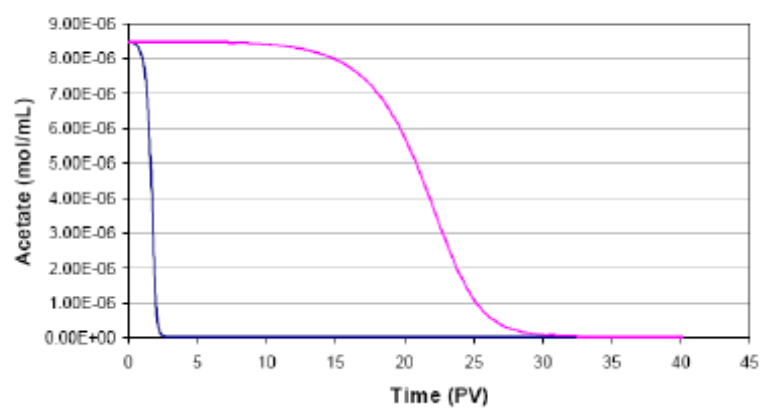

Fig. 7-Acetate concentration history at the centre of the last grid block of the PARSSIM simulation grid with smaller values of maximum specific growth rate. The blue curve has a 13 orders smaller value, the pink curve 14 orders smaller. The behaviour is qualitatively similar to Fig. 6a. The values for the other Monod kinetics parameters used in this simulation were as Fig. 6. 


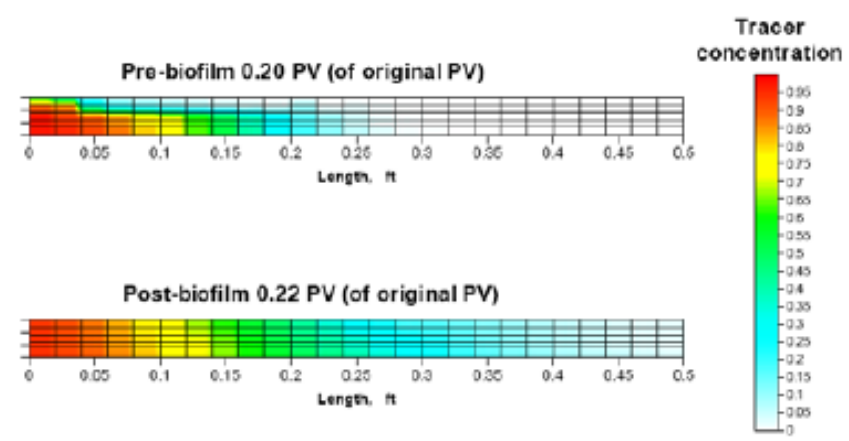

Fig. 8-Simulated concentration profiles before (above) and after (below) nutrient injection. These history-matched UTCHEM simulations (Gandler et al., 2006) confirm the diversion of flow from high permeability (lower half of domain) to low permeability areas (upper half of domain). The diversion of flow also confirms the growth of the biofilm and its ability to effectively divert flow from high permeability layers to lower permeability layers.

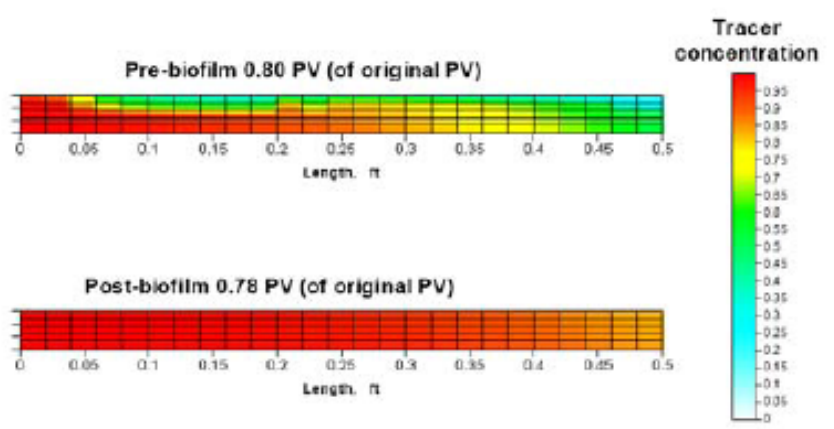

Fig. 9-Comparison of pre-biofilm and post biofilm tracer at a higher pore volume injected when biofilm has reached maturity. Concentration profiles confirm the diversion of flow from high permeability layers to low permeability layers.

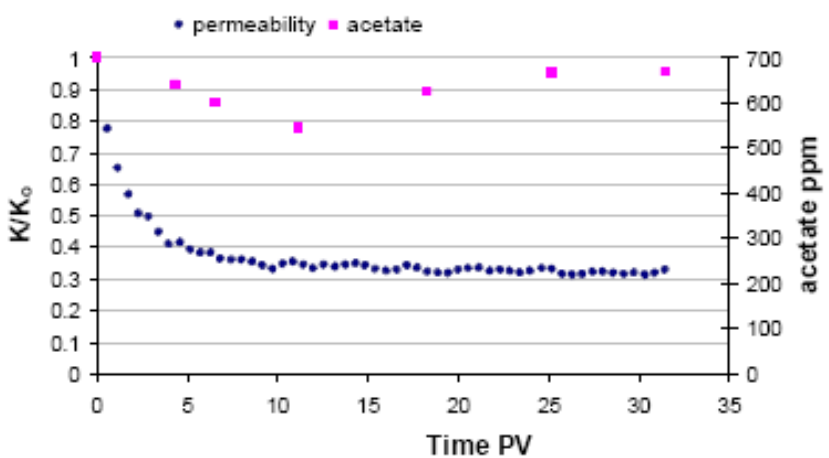

Fig. 10-Effluent acetate concentration and permeability reduction for homogeneous bead pack. The initial and injected concentration of acetate is $700 \mathrm{ppm}$. 


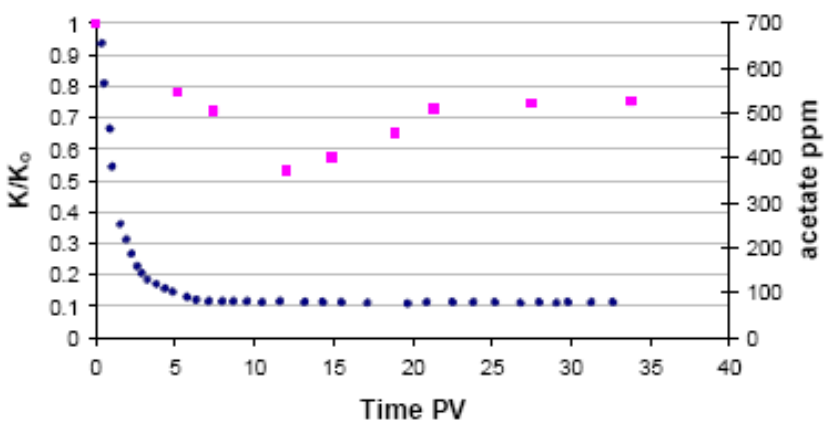

- permeability = acetate

Fig-11. Heterogeneous bead pack effluent and permeability reduction history (two layers of different permeability). The initial and injected concentration of acetate is $700 \mathrm{ppm}$.

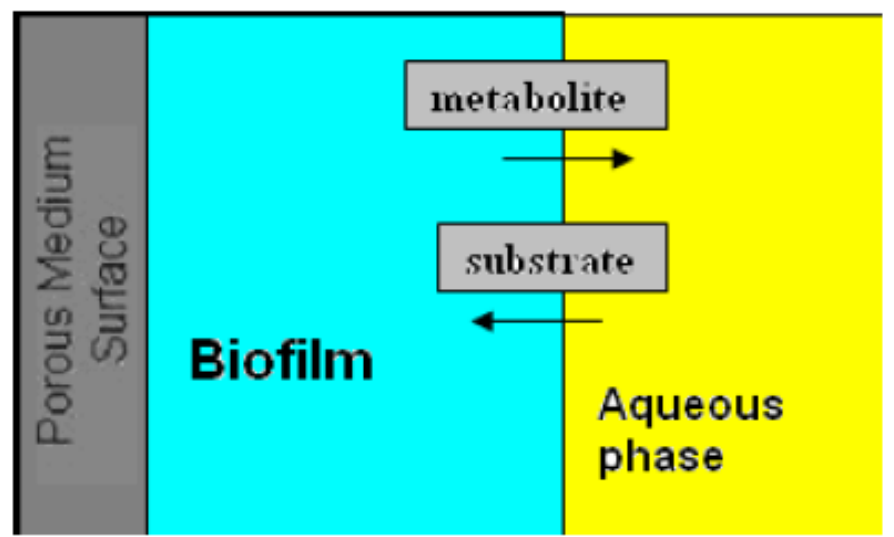

Fig. 12-A schematic of the diffusion process in biofilms. The substrate has to diffuse across the biofilms to reach the lower stack of cells in the biofilm. The time scale for diffusion can become longer than the reaction time, so that kinetics of substrate consumption differs within a porous medium than in a batch culture.

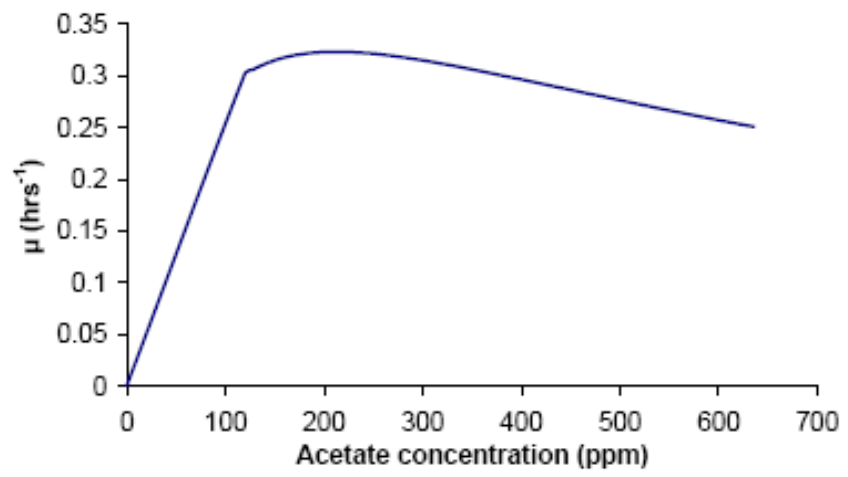

Fig. 13-Typical behavior of a specific growth curve in case of substrate inhibition. The specific growth rate increases to a maximum as acetate concentration increases, then decreases if the substrate concentration is increased further. 


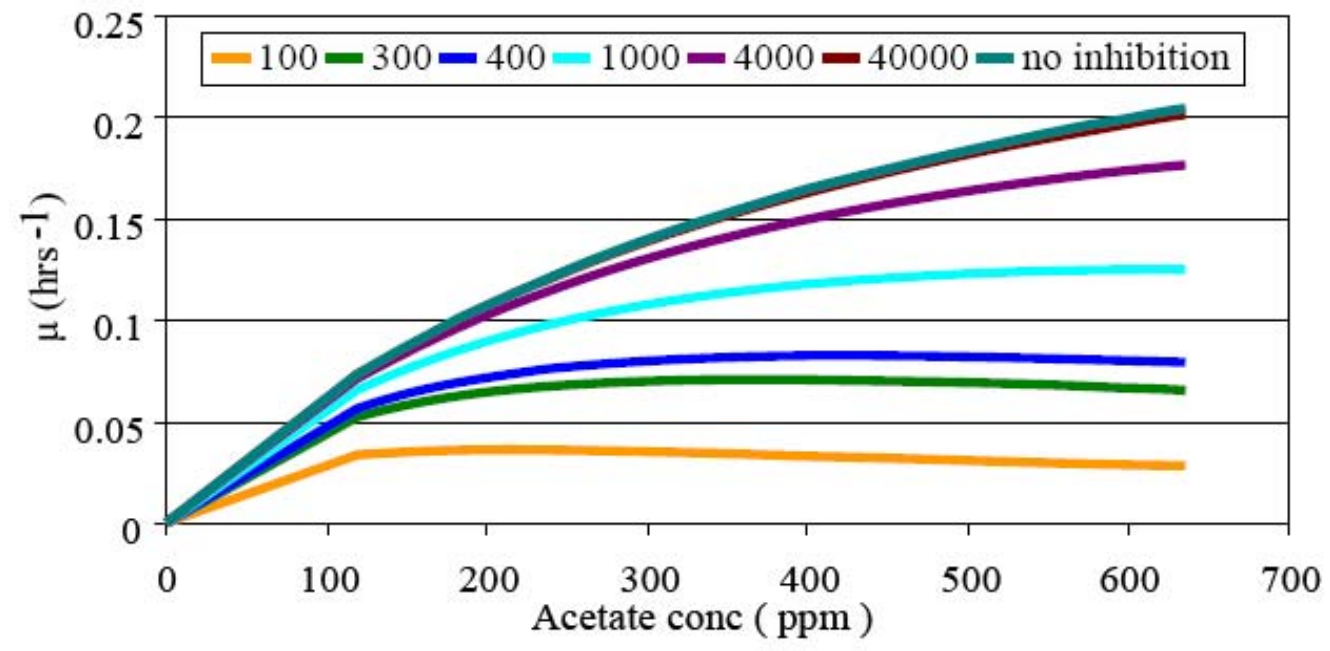

Fig. 14-Andrews and Noack model for substrate inhibition. The curves for 100,300 and $400 \mathrm{ppm}$ inhibition constant show a declining trend in microbial growth with increasing substrate concentration in batch experiments. 
SUPPLEMENTAL ANALYSIS OF SELF-LIMITING GROWTH MODEL

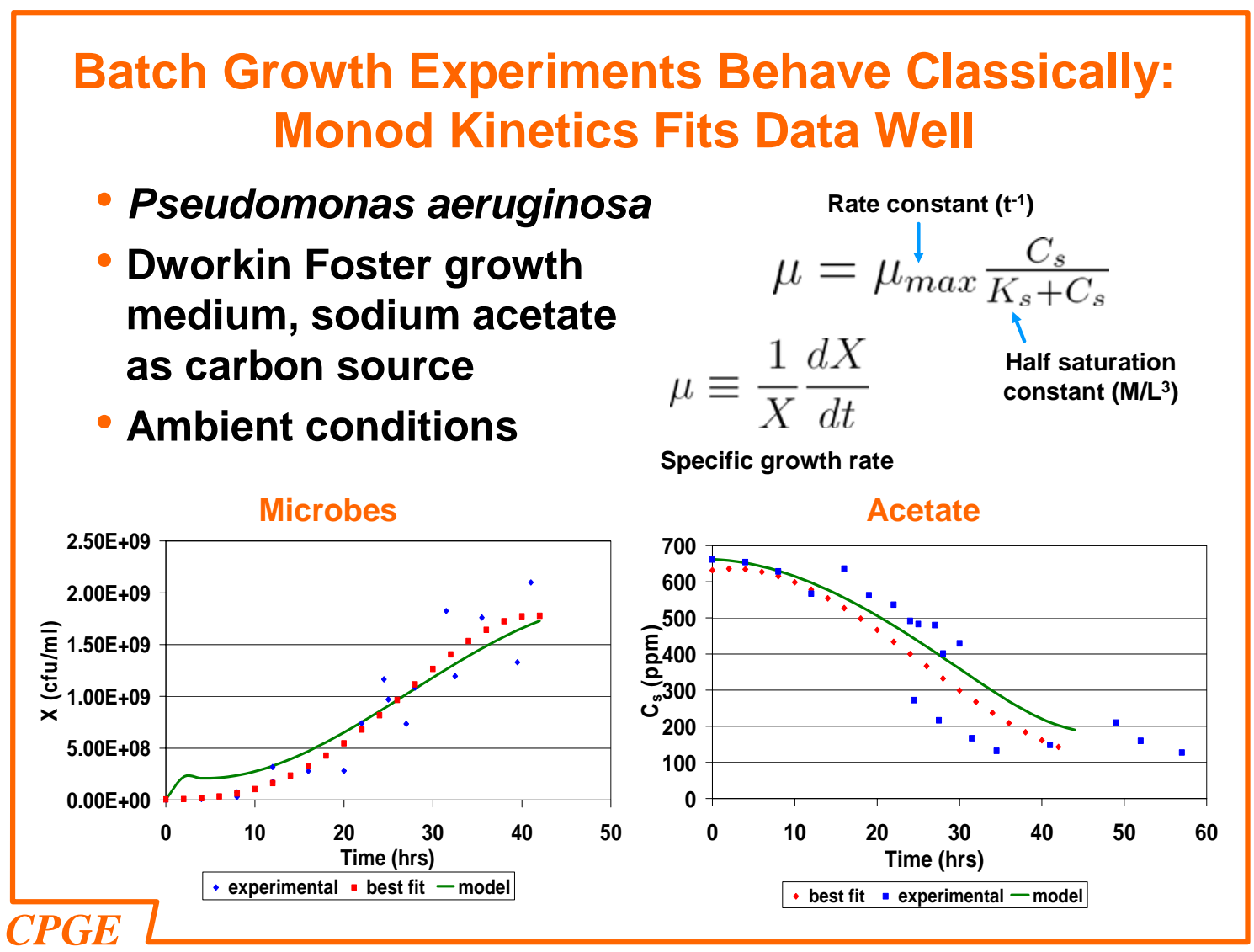

Our experiments in batch operating conditions using our model organism P. aeruginosa behave classically. The tradition Monod kinetics expression $\mu=\mu_{\max } \frac{C_{s}}{K_{s}+C_{s}}$ explains the microbe growth curve (left figure) and the nutrient consumption (right figure). 


\section{Coreflood Experiments in Homogeneous Columns Demand Revised Modeling}

- Homogeneous bead packs

- Increasing, then decreasing nutrient consumption

- Permeability decrease

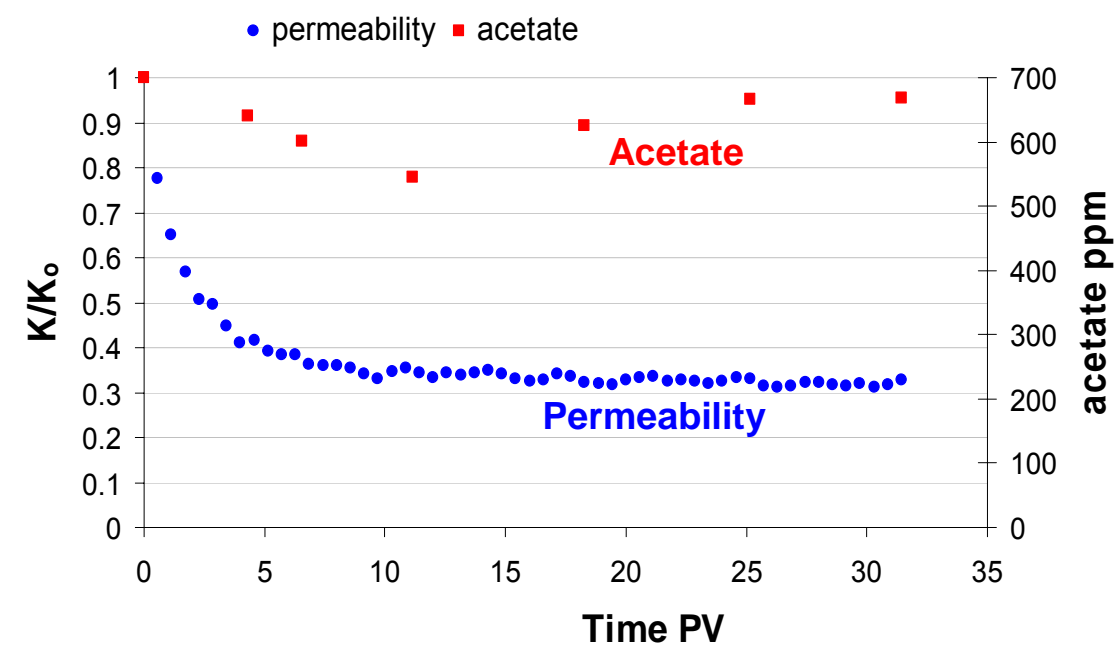

CPGE

However, corefloods in which the same model organism, P. aeruginosa, is grown within a porous medium, the uptake of nutrient cannot be explained by the same kinetics that explain the batch reaction. The acetate consumption within the core typically increases for ten PV of injection, then decreases. Consequently the effluent acetate concentration, shown above for a typical experiment, decreases, then increases. This cannot occur with the classical Monod expression.

The essential problem is that in a column flood, fresh nutrient is continually introduced. Unlike the batch reactor, there is no limit to growth caused by competition for dwindling food supply, at least near the inlet of the column.

The increase in effluent acetate concentration is associated with the leveling off of permeability. These two observations indicate that microbe growth has ceased. The fact that acetate concentration does not return to the injected level indicates that the microbes are still active. Evidently they have grown until space limitations force them to adopt a "resting" metabolic rate, rather than the "multiplying" metabolic rate. This requires a revision to the kinetics model for application to growth in the confined voids of a porous medium. 


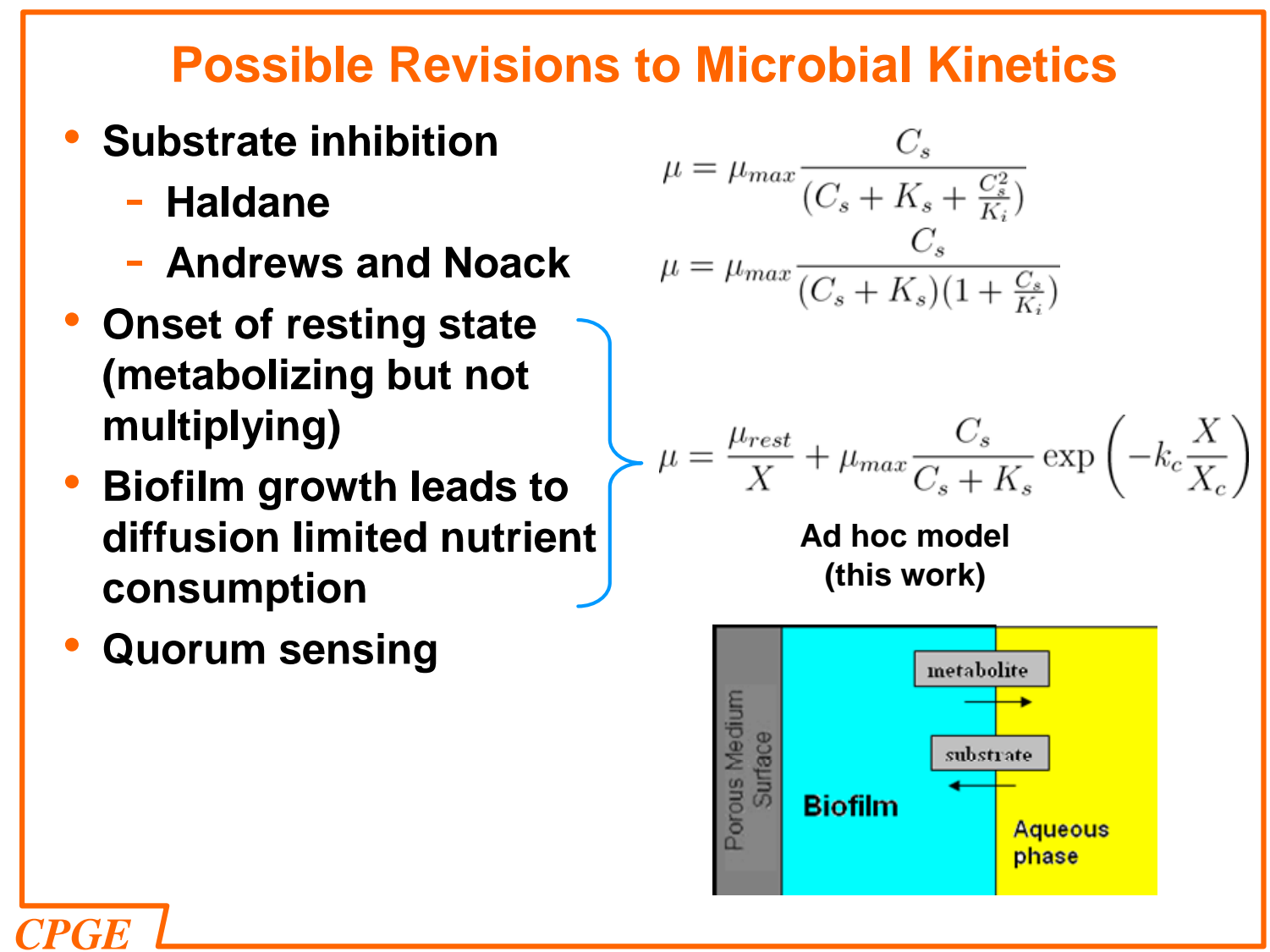

A variety of inhibition kinetics models have been proposed, e.g. by Haldane and by Andrews and Noack. Qualitatively, however, these cannot explain the behavior inferred in our columnflood experiments. Instead a new model is proposed, of the following form:

$$
\mu=\frac{\mu_{\text {rest }}}{X}+\mu_{\max } \frac{C_{s}}{C_{s}+K_{s}} \exp \left(-k_{c} \frac{X}{X_{c}}\right)
$$

This equation introduces a self-limiting behavior by means of the exponential term. As the microbe concentration $X$ increases beyond a critical value $X_{c}$ this term decreases rapidly, causing the nutrient consumption via classical Monod kinetics to decrease. The decrease is more rapid than the increase in microbe concentration, so this contribution to the specific growth rate $\mu$ to decrease asymptotically to zero.

This equation also introduces a resting metabolic rate, the first term on the right hand side of the equation. The term is constructed by dividing a nominal constant specific rate of nutrient consumption (assumed constant) by microbe concentration. This form leads to a constant consumption of nutrient. The value is small and thus is not noticeable during the growth phase. When growth ceases (second term vanishes), this background metabolic rate remains and becomes measurable. 


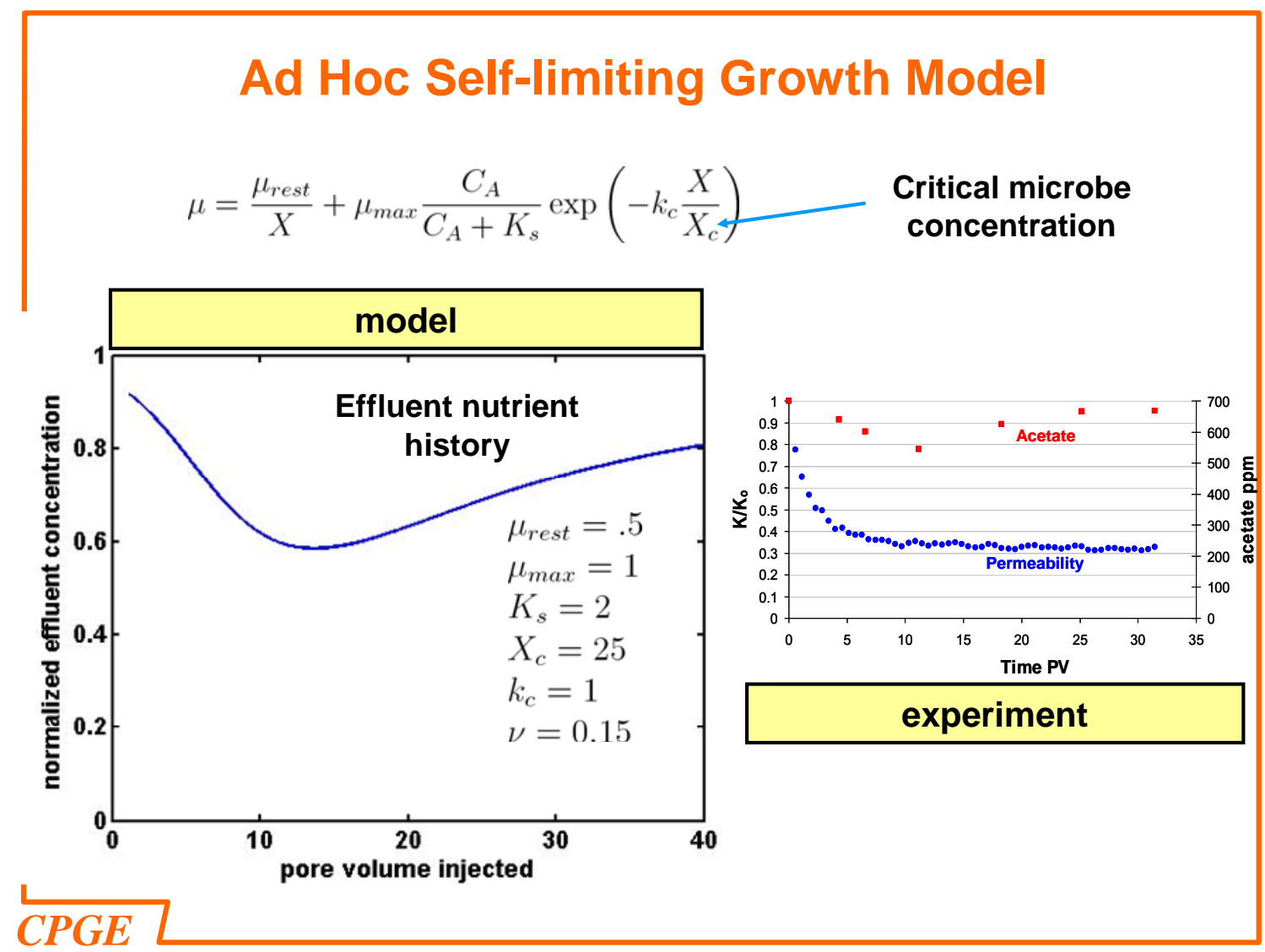

Evaluating the ad hoc model mathematically, we find an effluent nutrient concentration history (left figure) that is qualitatively similar to the observed effluent acetate concentration histories in the columnfloods (right figure). This provides some indication that modeling the transition from "growth phase" to "maintainence phase" is essential for correctly predicting nutrient uptake in a continuous flow setting (laboratory or field.) Since permeability reduction is only associated with the growth phase, this has obvious important implications for field deployment of the technology. The key unresolved issue at this point is the trigger for the transition to "maintainence" from "growth". It is not clear whether this is associated with the confinement of the microbes to small void space, or simply the consequence of biofilm growth inducing a diffusion-limited constraint on growth rate. 